PNNL-13186

UC-600

\title{
Tanks Focus Area Site Needs Assessment
} FY 2000

March 2000

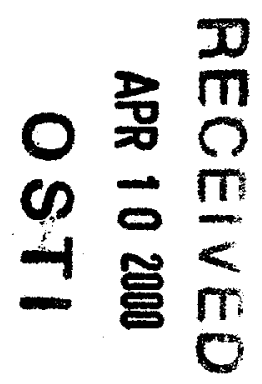

Prepared for

the U.S. Department of Energy

under Contract DE-AC06-76RLO 1830

Pacific Northwest National Laboratory

Richland, Washington 99352 


\section{DISCLAIMER}

This report was prepared as an account of work sponsored by an agency of the United States Government. Neither the United States Government nor any agency thereof, nor any of their employees, make any warranty, express or implied, or assumes any legal liability or responsibility for the accuracy, completeness, or usefulness of any information, apparatus, product, or process disclosed, or represents that its use would not infringe privately owned rights. Reference herein to any specific commercial product, process, or service by trade name, trademark, manufacturer, or otherwise does not necessarily constitute or imply its endorsement, recommendation, or favoring by the United States Government or any agency thereof. The views and opinions of authors expressed herein do not necessarily state or reflect those of the United States Government or any agency thereof. 


\section{DISCLAIMER}

Portions of this document may be illegible in electronic image products. Images are produced from the best available original document. 


\section{Executive Summary}

The Tanks Focus Area's (TFA's) mission is to deliver integrated technical solutions that enable tank waste remediation to be successful across the Department of Energy (DOE) complex. To do this, the TFA

- Brings together users and technical experts to define and execute the mission

- Integrates the work across the sites and other funding organizations

- Builds teams of users and providers to deliver and deploy technical solutions.

The TFA uses a systematic process for developing its annual program that draws from the tanks technology development needs expressed by five DOE tank waste sites - Hanford Site, Idaho National Engineering and Environmental Laboratory (INEEL), Oak Ridge Reservation (ORR), Savannah River Site (SRS), and West Valley Demonstration Project (WVDP). During the past year, the Fernald Site joined the TFA family to develop mutually beneficial information exchanges and lessons learned. Although Fernald does not now have science and technology needs requiring TFA action, the TFA is eager to build its future relationship with Fernald to expand the TFA's ability to capitalize on successful technical solutions throughout the DOE complex.

The TFA's annual program development process is iterative and involves the following steps:

- Collection of site needs

- Needs analysis

- Development of technical responses and initial prioritization

- Refinement of the program for the next fiscal year

- Formulation of the Corporate Review Budget (CRB)

- Preparation of Program Execution Guidance (PEG) for the next FY

- Revision of the Multiyear Program Plan (MYPP).

This document describes the outcomes of the first phase of this process, from collection of site needs to the initial prioritization of technical activities. 
Table ES.1. Summary of Site Needs Submitted to the Tanks Focus Area

\begin{tabular}{|l|c|c|c|c|c|c|}
\hline & Hanford & INEEL & ORR & SRS & WVDP & Total \\
\hline Safety & 4 & 2 & 1 & 2 & 1 & 10 \\
\hline Characterization & 3 & 8 & 1 & 1 & 1 & 14 \\
\hline Pretreatment & 2 & 13 & 2 & 4 & 0 & 21 \\
\hline Immobilization & 4 & 11 & 1 & 4 & 2 & 22 \\
\hline Retrieval & 8 & 1 & 2 & 6 & 2 & 19 \\
\hline Closure & 4 & 6 & 1 & 1 & 1 & 13 \\
\hline Total & 56 & 45 & 7 & 24 & 8 & 140 \\
\hline
\end{tabular}

Each site's Site Technology Coordination Group (STCG) was responsible for developing and delivering priority tank waste needs. The TFA was pleased to receive site needs in October November 1998, earlier than in previous fiscal years. A total of 140 site needs were received, an increase of 42 over the previous year. The needs were analyzed and integrated, where appropriate. Forty-eight distinct technical responses were drafted and prioritized. In addition, six strategic tasks were approved to compete for available funding in FY 2001 and FY 2002. The TFA matched each need to one or more of six functions: safety, characterization, pretreatment, immobilization, retrieval, and closure. A summary of the TFA's functional assignment of the needs is shown in Table ES.1.

To prioritize the technical responses, the TFA used four rating criteria:

- Broad-based benefit - This criterion rated whether the technical responses could satisfy needs at multiple sites (complex-wide impact).

- User commitment to deploy - The TFA assessed the user's commitment based on interest expressed in the needs description and present or future co-funding of development and/or deployment.

- Technical risk - This criterion considered the site needs priorities and waste stream risks related to a technical response.

- Other technical impact - The TFA considered a technical response's impacts on schedule, cost avoidance, and link to regulatory requirements.

Draft technical responses were prepared and provided to the TFA Technical Advisory Group for technical review, then to the TFA Site Representatives and the TFA User Steering Group (USG) for their review and comment. These responses were discussed at a March 9, 2000, meeting where the TFA Management Team established the priority listing in preparation for input to the DOE Office of Science and Technology (OST) budget process. At the time of publication of this document, the TFA continues to finalize technical responses as directed by the TFA Management Team and clarify the intended work scopes for FY 2001 and FY 2002. Presently, the FY 2002 CRB is under development, reflecting the priorities established by the TFA Management Team. 
Each year the TFA takes a critical look at its needs assessment process to determine where to direct self-improvement efforts for the next year. Last year was the TFA's first full program development cycle under the "Focus Area-centered" concept. The Focus Area-centered concept calls for Focus Areas, such as the TFA, to lead the coordination and integration of all OST programs within its problem area. The TFA's primary program partners include the

- Characterization, Monitoring, and Sensor Technology (CMST) Crosscut Program

- Efficient Separations and Processing (ESP) Crosscut Program

- Robotics (RBX) Crosscut Program

- Industry Programs

- University Programs

- International Programs

- Accelerated Site Technology Deployment (ASTD) program

- Environmental Management Science Program (EMSP).

Since last year, the TFA began negotiating operating agreements with three Crosscutting program partners - CMST, ESP, and RBX. Memorandums of Understanding (MOUs) between the TFA and each of these three programs are being finalized.

Coordinating site needs analyses and technical response development with and between these programs continues to present a sizeable task, and the TFA appreciates the efforts of its partner programs to help meet the challenge. The requirement exists to better synchronize, at the DOE Environmental Management (EM) and Office of Science and Technology (OST) level, the scheduling of program development activities that culminate in the preparation and submission of draft budget documents in the March-April timeframe of each year.

Development and use of the Integrated Planning, Accounting, and Budgeting System (IPABS) continues to be a serious impediment to program development. More work is required during the next year to make the system less cumbersome, more reliable, and better integrated. Data quality issues remain, especially in waste stream linkages to site needs and technical risks existing in those waste streams. The TFA has taken on an added burden of helping identify to the sites where data inconsistencies exist. Although this is the second year that the IPABS system is being used to develop OST work package priorities, there continues to be great uncertainty about data quality, rating criteria, and system scheduling requirements leading up to work package prioritization.

The TFA continues to grow into its Focus Area-centered responsibilities in the basic science portion of its investment portfolio. While some progress was made during the last year in strengthening relationships with the Environmental Management Science Program (EMSP) staff, serious program development and execution issues still require significant attention. 
THIS PAGE INTENTIONALLY BLANK 


\section{Contents}

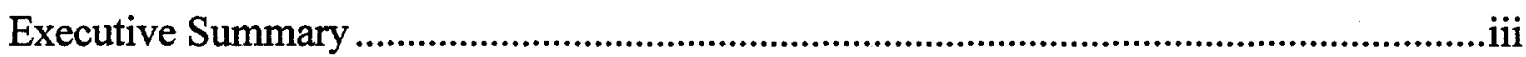

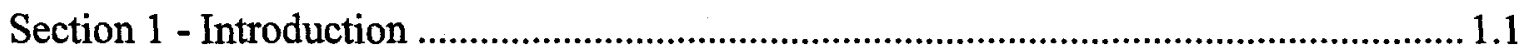

Section 2 - Site Needs Assessment and Technical Response Development Process......... 2.1

2.1 STCG Needs Submission and TFA Screen .................................................. 2.1

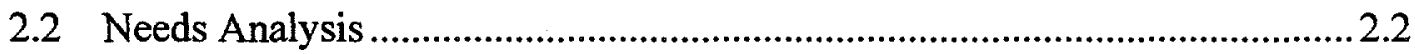

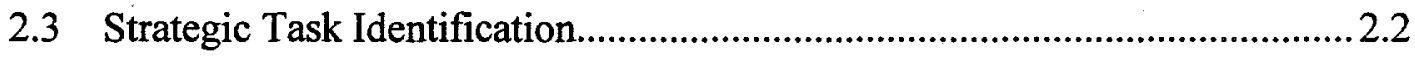

2.4 Technical Response Development ............................................................... 2.3

2.5 Technical Response Rating …...................................................................... 2.4

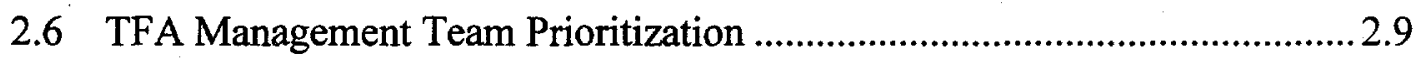

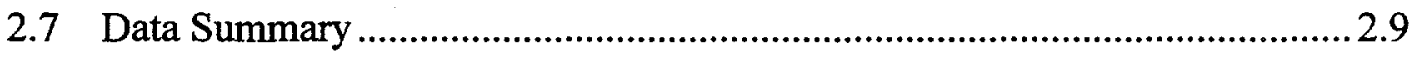

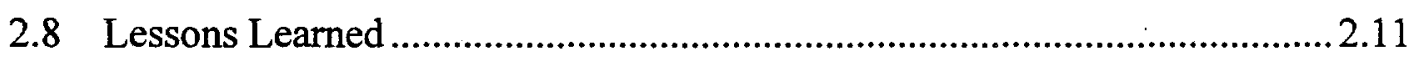

Section 3 - The Next Process Steps ............................................................................. 3.1

3.1 Finalize FY 2002 CRB Submittal ........................................................ 3.1

3.2 OST Work Package Prioritization for the FY 2002 CRB .............................. 3.1

3.3 Prepare and Submit FY 2001 Program Execution Documents ...................... 3.2

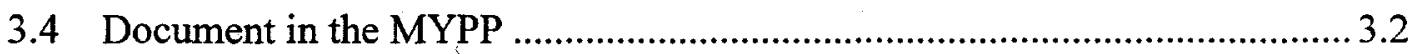

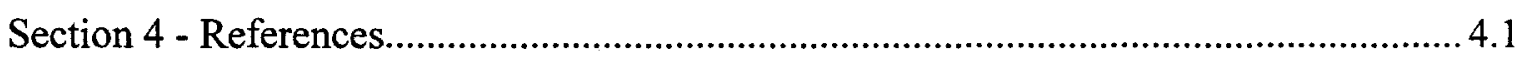

Appendix A - Site Needs Database .............................................................................. A.1 


\section{List of Figures}

1.1 FY 1999 Tanks Focus Area Technical Response Development Process............... 1.4

2.1 Tanks Focus Area Organization...................................................................2.2

\section{List of Tables}

ES.1 Summary of Site Needs Submitted to the Tanks Focus Area ...............................iv

$2.1 \quad$ Problem Element Structure .......................................................................... 2.5

2.2 Summary of Site Needs Submitted to the Tanks Focus Area ........................... 2.10

A.1 Tanks Focus Area Needs Submitted by Sites ............................................ A.2

A.2 Tanks Focus Area Site Needs Distributed within the Problem Element Structure ..................................................................... A.6

A.3 Tanks Focus Area FY 2001 - FY 2002 Integrated Priority Listing .................... A.12 


\section{Acronyms}

\begin{tabular}{|c|c|}
\hline ADMP & Advanced Design Mixer Pump \\
\hline AFD & adjustable frequency drive \\
\hline $\mathrm{Al}$ & aluminum \\
\hline ALARA & as low as reasonably achievable \\
\hline AMP-PAN & ammonium molybdophosphate-polycrylonitrile \\
\hline ASTD & Accelerated Site Deployment Program \\
\hline BNFL & British Nuclear Fuels, Limited, Inc. \\
\hline CAA & Clean Air Act \\
\hline CERCLA & Comprehensive Environmental Response, Compensation and Liability Act \\
\hline CFR & Code of Federal Regulations \\
\hline $\mathrm{CHG}$ & CH2M Hill Hanford Group \\
\hline CIF & Consolidated Incineration Facility \\
\hline CLE & contaminated large equipment \\
\hline CMST & Characterization, Monitoring, and Sensor Technology Program \\
\hline CPES & Chemical Process Evaluation System \\
\hline $\mathrm{Cr}$ & chromium \\
\hline CRB & Corporate Review Budget \\
\hline Cs & cesium \\
\hline CSSF & Calcine Solids Storage Facility \\
\hline CST & crystalline silicotitanate \\
\hline CTS & Concentrate Transfer System \\
\hline $\mathrm{D} \& \mathrm{D}$ & decontamination and decommissioning \\
\hline $\mathrm{DEQ}$ & (Idaho's) Department of Environmental Quality \\
\hline DOE & U.S. Department of Energy \\
\hline DOE-ID & U.S. Department of Energy's Idaho Operations Office \\
\hline DST & double-shell tank \\
\hline DWPF & Defense Waste Processing Facility \\
\hline ECR & effective cleaning radius \\
\hline EIS & Environmental Impact Statement \\
\hline EM & Office of Environmental Management \\
\hline EM-30 & Office of Waste Management \\
\hline EM-50 & Office of Science and Technology \\
\hline EMSP & Environmental Management Science Program \\
\hline $\mathrm{EN}$ & electrochemical noise \\
\hline EPA & U.S. Environmental Protection Agency \\
\hline ESP & Efficient Separations and Processing Program \\
\hline ESP & Environmental Simulation Program \\
\hline ESW & enhanced sludge washing \\
\hline
\end{tabular}


fiscal year

$\begin{array}{ll}\text { HAW } & \text { high activity waste } \\ \text { HEPA } & \text { high-efficiency particulate air } \\ \text { Hg } & \text { mercury } \\ \text { HLW } & \text { high-level waste } \\ \text { HLWSC } & \text { High-Level Waste Steering Committee }\end{array}$

ILAW immobilized low activity waste

INEEL Idaho National Engineering and Environmental Laboratory

INTEC Idaho Nuclear Technology and Engineering Center

IPABS Integrated Planning, Accounting, and Budgeting System

\begin{tabular}{|c|c|}
\hline LAW & low activity waste \\
\hline LDUA & Light Duty Utility Arm \\
\hline LDR & land disposal restriction \\
\hline LET\&D & liquid effluent treatment and disposal \\
\hline LLW & low-level waste \\
\hline LMITCO & Lockheed-Martin Idaho Technology Company \\
\hline MAXT & maximum achievable control technology \\
\hline MOU & Memorandum of Understanding \\
\hline MPC & Main Process Cell \\
\hline MYPP & Multiyear Program Plan \\
\hline NDE & non-destructive examination \\
\hline NEPA & National Environmental Policy Act \\
\hline NGLW & newly-generated liquid waste \\
\hline NON & Notice of Noncompliance \\
\hline NTS & Nevada Test Site \\
\hline ORNL & Oak Ridge National Laboratory \\
\hline ORR & Oak Ridge Reservation \\
\hline ORWBG & Old Radioactive Waste Burial Ground \\
\hline ORP & DOE's Office of River Protection \\
\hline OST & DOE's Office of Science and Technology \\
\hline PBS & project baseline summary \\
\hline PCCS & Product Composition Control System \\
\hline PCT & product consistency test \\
\hline PCB & polychlorinated biphenyl \\
\hline PE & problem element \\
\hline PEG & Program Execution Guidance \\
\hline PEWE & Process Evaporative Waste Evaporator \\
\hline PHMC & Project Hanford Management Contractor \\
\hline
\end{tabular}


PIC products of incomplete combustion

PNNL Pacific Northwest National Laboratory

RBX Robotics Crosscut Program

RCRA Resource Conservation and Recovery Act

REDOX reduction-oxidation

RL DOE's Richland Operations Office

ROD Record of Decision

RPP River Protection Project

SBW sodium-bearing waste

$\mathrm{Sr}$ strontium

SRS Savannah River Site

SST single-shell tank

STCG Site Technology Coordination Group

TAG (TFA's) Technical Advisory Group

Tc technetium

TFA Tanks Focus Area

TIM (TFA's) Technology Integration Manager

TRU transuranic (waste)

TRUEX transuranic extraction

TSCA Toxic Substances Control Act

TTP technical task plan

TWRS Tank Waste Remediation System

UDS undissolved solids

UNEX universal solvent extraction

USG (TFA's) User Steering Group

WAC Waste Acceptance Criteria

WAPS Waste Acceptance Product Specifications

WASRD Waste Acceptance System Requirements Document

WBS

WIPP

work breakdown structure

WMA waste management area

WVDP West Valley Demonstration Project 


\section{THIS PAGE INTENTIONALLY BLANK}




\section{Section 1 - Introduction}

This report documents the process used by the Tanks Focus Area (TFA) to analyze and develop responses to technology needs submitted by five major U.S. Department of Energy (DOE) sites with radioactive tank waste problems, and the initial results of the analysis. The sites are the Hanford Site, Idaho National Engineering and Environmental Laboratory (INEEL), Oak Ridge Reservation (ORR), Savannah River Site (SRS), and West Valley Demonstration Project (WVDP). During the past year, the TFA established a link with DOE's Fernald site to exchange, on a continuing basis, mutually beneficial technical information and assistance.

This is the sixth edition of the TFA site needs assessment. As with previous editions, this edition serves to provide the basis for accurately defining the TFA program for the upcoming fiscal year (FY), and adds definition to the program for up to 4 additional outyears. Therefore, this version distinctly defines the FY 2001 program and adds further definition to the FY 2002 - FY 2005 program. Each year, the TFA reviews and amends its program in response to site users' science and technology needs.

Overall, the TFA's annual program development cycle involves the

- Collection of site needs

- Needs analysis

- Development of technical responses and initial prioritization

- Refinement of the program for the next FY

- Formulation of the Corporate Review Budget (CRB)

- Preparation of Program Execution Guidance (PEG) for the next FY

- Revision of the multiyear program plan (MYPP).

This document describes the TFA's process of collecting site needs, analyzing them, and developing technical responses to the needs. It also summarizes the information captured within the TFA needs database, including information provided by the five major DOE sites with tank waste problems. The technical scope of the TFA's 5-year program will be defined in detail with the publication of the companion to this document, the MYPP, in September 2000 . 
The TFA goal remains unchanged -- to provide integrated solutions that will accelerate safe and cost-effective cleanup and closure of DOE's tank system. At the five major tank waste sites, the TFA focuses on the $282 \operatorname{tanks}^{1}$ that contain approximately $380,000 \mathrm{~m}^{3}$ of high-level waste (HLW), low-level waste (LLW), and transuranic (TRU) waste. There are a number of smaller tanks at these sites that are outside of the TFA's purview at this time. The varying tank structure, construction, and capacity, as well as the different waste types themselves, provide an extraordinary challenge to the formation of an integrated tanks science and technology program. Multiple programmatic, institutional, and regulatory issues across the five sites add to the complex-wide challenge of remediation.

The overall TFA program objective is to deliver a tank science and technology program that reduces the current cost and the technical, operational, and safety risks of tank remediation. The TFA continues to enjoy close, cooperative relationships with each site. During the past year, the Fernald Site joined the TFA in a role that emphasizes exchange of experiences and information for mutual benefit. In the upcoming year, the TFA will work with Fernald to identify targets of opportunity that capitalize on Fernald's successes and address the breadth of problems faced by the TFA complex-wide.

The TFA continues to emphasize technical assistance and integration activities. These activities are essential, especially considering the dynamic environment at several sites. New or amended site needs frequently arise, requiring the TFA to be prepared not only to amend its program in response, but also to help the sites arrive at the best technical approach to solve revised site needs. Additionally, as the results of technology development are not $100 \%$ guaranteed, the TFA must work with the sites to find appropriate alternative solutions if technology development and deployment results do not meet expectations.

Since its inception, the TFA continues to cite four tanks technology program attributes essential for TFA success. These attributes guide the TFA's service to the user, such that the program is

- Applicable - addresses users' needs and can be implemented within budget, schedule, and regulatory constraints. The TFA uses a consensus-driven site needs collection and technical response process that enhances a deeper understanding of the interrelationships of the needs. Through this process, the TFA developed a priority listing of FY 2001 and FY 2002 proposed activities in accordance with representatives from all five major tank waste sites.

- Integrated - leverages relevant activities across the DOE Office of Environmental Management (EM) system, across the DOE complex, and beyond. The TFA is part of a science and technology network formed within the Office of Science and Technology (OST) and Environmental Management (EM) at each site. The awareness of related work between sites and focus areas continues to grow. The TFA fosters this awareness through leveraging opportunities. Under the "Focus Area-centered" concept, the TFA is making a

\footnotetext{
${ }^{1}$ In 1997, two of these tanks were closed.
} 
concerted effort to more fully integrate resources available from all other Office of Science and Technology (OST) activities. As of the date of this document, the TFA is negotiating operating agreements with three OST Crosscutting Programs [Efficient Separations and Processing Program (ESP), Characterization, Monitoring, and Sensor Technology Program (CMST), and Robotics Program].

- Acceptable - has broad involvement of key stakeholders and incorporates expertise from outside the laboratory system (e.g., from industry and universities $\}$ as appropriate. The TFA has made special efforts to involve stakeholders. These stakeholders include the Site Technology Coordination Groups (STCGs), and the TFA User Steering Group (USG).

- Accountable - performs within budget, on schedule, and produces a clear benefit. The TFA continues to execute its mission with a high degree of accuracy, both fiscally and within milestone schedules. As a result, the TFA maintains the confidence of site users.

The TFA accomplishes its objective by executing an iterative approach to program development that involves site users and stakeholders through the STCGs at each site. The needs assessment forms the basis for TFA program definition. As previously noted, the TFA's program development cycle begins with the collection of site needs and ends with the publication of the MYPP. This site needs assessment describes the TFA's efforts through the first part of this cycle, from site needs collection through the development of technical responses and their initial prioritization. The TFA uses six steps to accomplish the first part of this cycle, which are listed below and depicted in Figure 1.1:

- STCG needs submission and TFA screen

- Needs analysis

- Strategic task identification

- Technical response development

- Response evaluation

- TFA Management Team prioritization.

The TFA Management Team approved the TFA task prioritization for FY 2001 and FY 2002 on March 9,2000. Work is underway to finalize the technical responses developed earlier and to prepare the FY $2002 \mathrm{CRB}$. The final technical responses will form the basis for Program Execution Guidance (PEG) development required for execution of the FY 2001 program.

Section 2 of this site needs assessment describes the TFA's process in reaching this point, from needs collection and analysis to task prioritization. Section 3 describes follow-on program development activities the TFA will use to complete this year's program development process cycle. Appendix A contains a summary of the needs submitted by the sites and the TFA's initial disposition of them through technical responses and prioritization. 

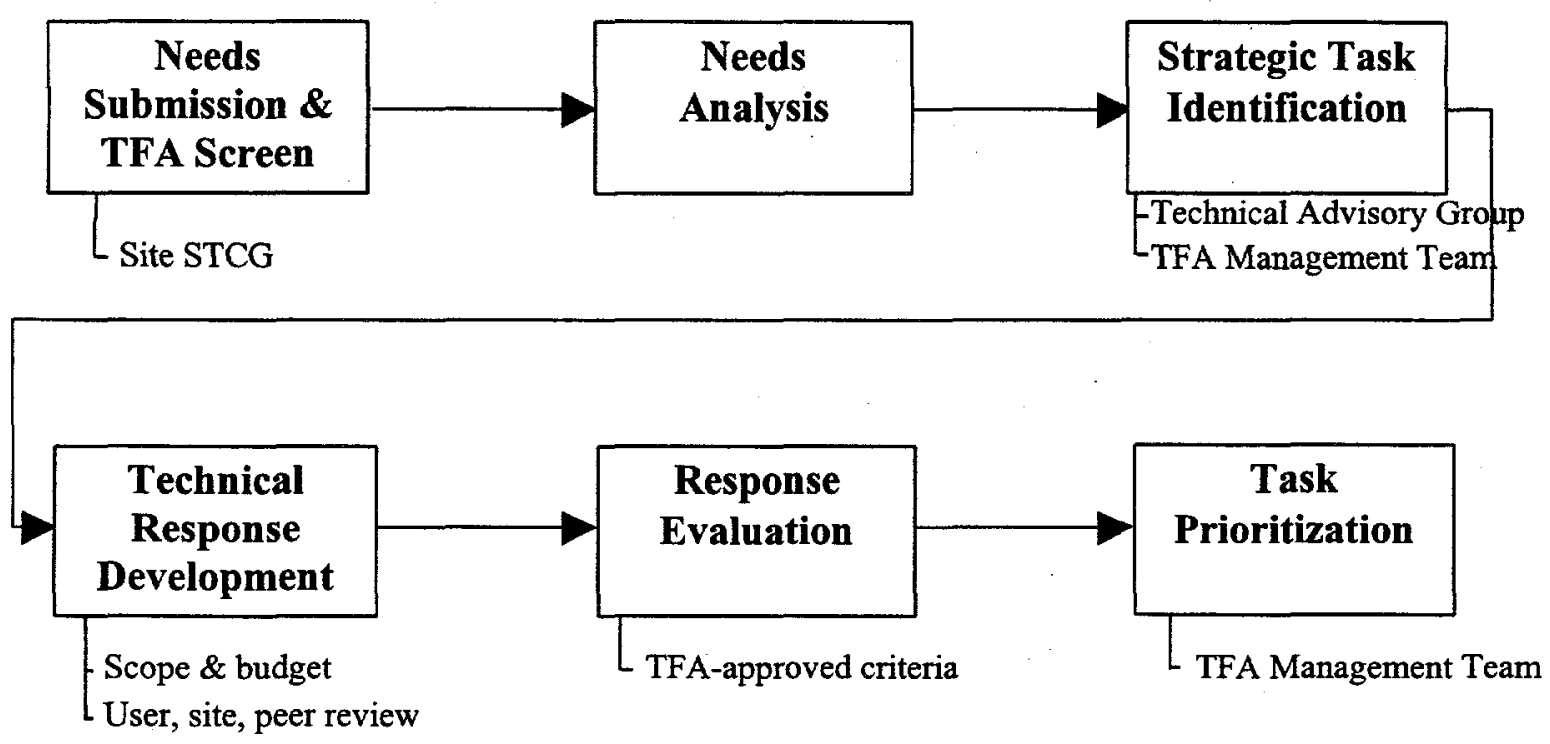

Figure 1.1. FY 2000 Tanks Focus Area Technical Response Development Process 


\section{Section 2 - Site Needs Assessment and Technical Response Development Process}

The TFA continues to enjoy a general endorsement of its program development process from its site user community. Two changes were made this year to fine-tune the TFA's proven process of program development. First, the TFA amended its approach to strategic task development, review, and prioritization. Second, minor changes were made to the TFA's prioritization criteria. These changes reflect the TFA's emphasis in:

- A highly participitative, consensus-based, user-driven program

- The importance of both near-term and longer-term problems and solutions

- Technical response prioritization consistent with DOE's Work Package Ranking System.

The program development process steps are (refer Figure 1.1)

- STCG needs submission and TFA screen

- Needs analysis

- Strategic task identification

- Technical response development

- Response evaluation

- TFA Management Team prioritization.

\subsection{STCG Needs Submission and TFA Screen}

The tank waste sites submitted their science and technology development needs from October - December 1999. Each site uses its own internal process to determine and prioritize site needs as necessary. The standardized site needs template again proved helpful in communicating and understanding the needs. The TFA's Site Representatives were essential in communicating the needs from the sites to the TFA. (See Figure 2.1, Tanks Focus Area Organization.) This year, the sites, in general, communicated their science and technology needs statements earlier than in any previous year. The TFA appreciated these earlier submissions, which provided additional time to integrate the information with other OST program activities.

Each need was subjected to an initial needs screening. The screening assessed whether or not the need and possible technical response

- Was within the TFA mission area

- Required a technology development component

- Development, first-time hot demonstration or deployment, re-engineering, etc., was required

- Technology was available, and no technology development was required

- Was technically feasible (schedule or cost). 


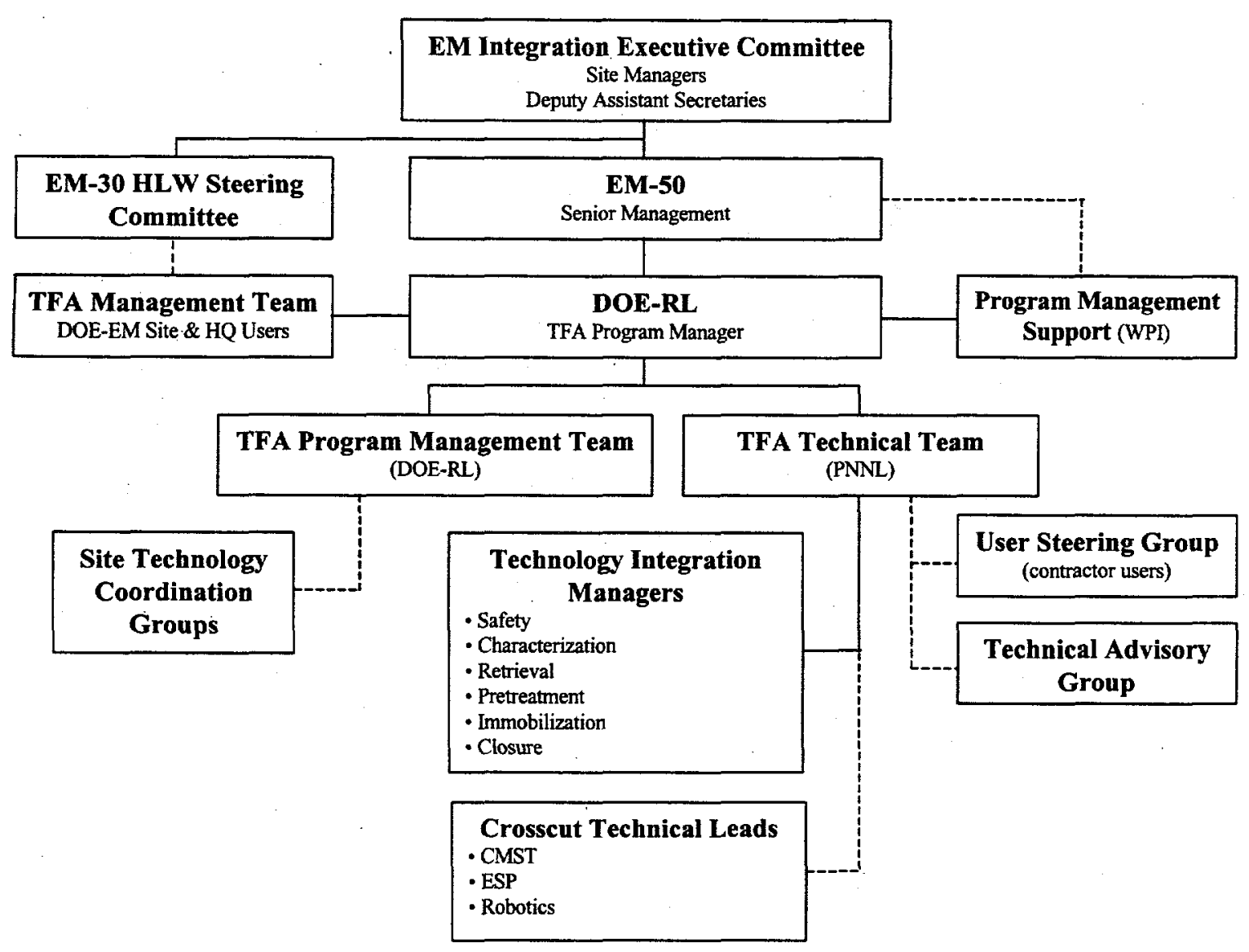

Figure 2.1. Tanks Focus Area Organization

\subsection{Needs Analysis}

The TFA analyzed each site need that passed through the screening criteria. This analysis served to familiarize the TFA with the general scope of site needs. The TFA worked interactively with the sites to better understand the problem to be solved, required performance specifications, timing of the technical solution, integration of functional interfaces (e.g., between pretreatment and immobilization), and interfaces with other OST programs.

\subsection{Strategic Task Identification}

Focusing predominately on the analysis of site-submitted needs, the TFA identified needs whose solutions would be strategic in nature to the TFA. Additionally, the TFA identified technology "gaps" that became apparent in the needs analysis, or that were identified through other TFA processes, such as technology interface workshops. The TFA Technical Advisory Group (TAG) provided advice and guidance on the identification and scope of proposed strategic tasks. The TFA submitted these issues for consideration and review by its Management Team. The Management Team either voiced no objection to the development 
of a technical response to these issues to be included within the TFA list of needs, or determined that the issue merited no further TFA consideration.

The TFA developed and refined its own definition of a strategic task. The following points define a TFA strategic task

- Pursues a problem identified within a site baseline, but not currently being addressed. This problem would be longer-term and may otherwise go unsatisfied due to budget limitations and priority. An official need may or may not have been submitted by the STCG of a specific site. Successful TFA response to the need may result in

- Accelerated schedule

- Risk reduction (programmatic or technical)

- Establishment of a technical or programmatic basis that drives near-term related baseline efforts.

- Resolves a technical roadblock or problem that has recently been identified. This problem may be near- or long-term in nature, and may or may not be associated with baseline technologies or flowsheets. This problem may be identified by the TFA or external reviewers, rather than officially submitted as a need by a specific site.

Satisfaction of this need may result in

- Prevention of recently identified problems

- Technical contingency through identification of another viable technical approach

- Risk reduction (programmatic or technical).

- Effects a change to a baseline (alternative). The problem could be near-term and may require that the TFA leverage other programs. An official need may or may not have been submitted by a site. Successful response to the need may result in

- Mortgage reduction

- Risk reduction (programmatic or technical).

The TFA has secured wide user support for the concept of selective identification and funding of strategic tasks, and identified six strategic tasks for initiation in FY 2001.

\subsection{Technical Response Development}

The TFA developed technical responses to all needs passing through the screening criteria. Those needs screened out were coordinated with the submitting site for further disposition. Some needs were screened out as potentially outside of the TFA mission area. These needs may best be addressed within a different OST program, such as another focus area. In such cases, the TFA interacts with the other programs and informs the submitting site STCG of any need identified as such in this process. 
The responses were prepared by the Technical Team (see Figure 2.1) and submitted to the TAG, USG, and Management Team for review and comment. To the maximum extent possible, the TFA integrated responses to similar needs. Also, the TFA was careful to take advantage of other OST funding sources to maximize leveraging opportunities.

The TFA uses an established standard framework to begin its annual program planning process. This framework groups similar or related site needs and the TFA's technical responses, allowing for technical integration across functions to solve specific problems, as opposed to consolidating needs by technical focus. This activity begins the transition from needs collection and analysis to TFA program development. The results of the program development process will be addressed in the upcoming revision to the MYPP scheduled for publication in September 2000.

To establish and maintain this program planning framework, the TFA uses its problem element structure. The problem elements

- Provide an updated method to logically group site needs and TFA technical responses

- Assist in sequencing and scheduling integrated technical solutions

- Identify the problem elements and the needs within them as baseline, enhancements, or alternatives.

The TFA problem element structure appears in Table 2.1.

\subsection{Technical Response Rating}

The TFA rated each technical response for use in funding decisions based on approved task selection criteria. Technical responses rated above the anticipated funding line are known as "core" tasks and generally form the basis for "target" budget funding levels. Selected technical responses below the funding line may be considered for TFA funding if they were previously identified as a strategic task. These strategic tasks will be highlighted for Management Team review and prioritization with rationale describing the benefits of investments relative to the TFA's strategic intent.

The TFA studied each need and developed draft integrated technical responses. As necessary, the TFA contacted the specific need technical point of contact for further clarification. From mid-January through early-March 2000, the TFA prepared an initial draft response for each need. The composite set of technical responses was rated against criteria intended to rank them for further program development activities. The criteria included the following:

- Broad-based benefit

- User commitment to deploy

- Technical risk

- Other technical impact. 
Table 2.1. Problem Element Structure

\begin{tabular}{ll} 
PE\# & Problem Element \\
\hline 1.1 & Store Waste \\
1.1 .1 & Extend Tank Life \\
1.1 .1 .1 & Monitor Tank Integrity/Avoid \\
& Corrosion \\
1.1.1.3 & Remediate Loss of Tank \\
& Integrity \\
1.1 .2 & Ventilate Tanks \\
1.1 .3 & Characterize Waste \\
1.1 .3 .1 & Characterize Waste In Situ \\
1.1 .3 .2 & Sample Waste \\
1.1 .3 .3 & Analyze Waste \\
1.1 .4 & Reduce Waste Volume \\
1.1 .4 .1 & Reduce Source Streams \\
1.1 .4 .2 & Reduce Recycle Streams \\
1.2 & Process Waste \\
1.2 .1 & Retrieve Waste \\
1.2 .1 .1 & Deploy Equipment \\
1.2 .1 .2 & Mobilize Bulk and Heel Wastes \\
1.2 .1 .4 & Transfer Waste \\
1.2 .1 .5 & Detect and Mitigate Leaks \\
1.2 .1 .6 & Monitor and Control Retrieval \\
& Process \\
1.2.1.7 & Integrate Retrieval and \\
& Pretreatment Technology \\
1.2.1.8 & Systems \\
1.2 .2 & Mobilize Heel \\
1.2 .2 .1 & Calcine Waste \\
1.2 .2 .2 & Dissolve Waste \\
1.2 .2 .3 & Prepare Retrieved Waste for \\
& Transfer and Pretreatment \\
1.2 .2 .4 & Clarify Liquid Stream \\
1.2 .2 .5 & Remove Radionuclides \\
1.2 .2 .6 & Integrate Pretreatment and LLW \\
& Immobilization Technology \\
1.2.2.7 & Systems \\
1.2 .2 .8 & Process Sludge \\
& Immobire Pretreated Waste for \\
&
\end{tabular}

\begin{tabular}{|c|c|}
\hline PE\# & Problem Element \\
\hline 1.2.2.9 & $\begin{array}{l}\text { Monitor and Control } \\
\text { Pretreatment Process }\end{array}$ \\
\hline 1.2 .3 & Immobilize Waste \\
\hline 1.2.3.1 & Process LLW \\
\hline 1.2.3.1.1 & $\begin{array}{l}\text { Monitor and Control LLW } \\
\text { Immobilization Process }\end{array}$ \\
\hline 1.2.3.1.2 & Prepare LLW Feed \\
\hline 1.2.3.1.3 & Immobilize LLW Stream \\
\hline 1.2.3.1.4 & Treat LLW Offgas \\
\hline 1.2.3.1.5 & Dispose of LLW \\
\hline 1.2 .3 .2 & Process HLW \\
\hline 1.2.3.2.1 & $\begin{array}{l}\text { Monitor and Control HLW } \\
\text { Immobilization Process }\end{array}$ \\
\hline 1.2.3.2.2 & $\begin{array}{l}\text { Prepare Secondary Waste from } \\
\text { Pretreatment }\end{array}$ \\
\hline 1.2 .3 .2 .3 & Prepare Sludge Feed \\
\hline 1.2.3.2.4 & Immobilize HLW Stream \\
\hline 1.2.3.2.5 & Treat HLW Offgas \\
\hline 1.3 & $\begin{array}{l}\text { Store Waste Forms and Close } \\
\text { Tanks }\end{array}$ \\
\hline 1.3.1 & Close Tanks \\
\hline 1.3.1.1 & Monitor Tank \\
\hline 1.3.1.2 & Characterize Heels \\
\hline 1.3.1.3 & Define Closure Criteria \\
\hline 1.3.1.4 & Treat Supernate in Place \\
\hline 1.3.1.5 & Treat Heel in Place \\
\hline 1.3.1.6 & Detect Leaks \\
\hline 1.3.1.7 & Stabilize Tank for Closure \\
\hline 1.3.1.8 & Monitor Site \\
\hline 1.3 .2 & Dispose of LLW \\
\hline 1.3.2.1 & Monitor LLW for Acceptance \\
\hline 1.3.2.2 & $\begin{array}{l}\text { Determine Performance of } \\
\text { Waste Form }\end{array}$ \\
\hline 1.3.2.3 & Provide Disposal System \\
\hline 1.3.3 & Store and Dispose HLW \\
\hline 1.3.3.1 & Provide Interim Storage HLW \\
\hline 1.3.3.2 & Provide Shipping Facilities \\
\hline 1.3.3.3 & Monitor HLW for Acceptance \\
\hline 1.4 & $\begin{array}{l}\text { Decontamination and } \\
\text { Decommissioning }\end{array}$ \\
\hline
\end{tabular}


Broad-Based Benefit - This criterion addressed the potential complex-wide benefit of a technical response.

High: Two or more different site STCG-submitted needs with strong interest in a single, integrated response. Note: "strong interest" means site interest is confirmed with the TFA Site Representative and USG member.

\section{High to Medium:}

- High/Medium: One STCG-submitted need; two or more sites with strong interest where resulting hardware or data would directly benefit.

- Medium/High: One STCG-submitted need; one site with strong interest where resulting hardware or data would directly benefit.

- Medium: One STCG-submitted need; one site with strong interest where resulting hardware or data would indirectly benefit; or one STCG-submitted need that may be satisfied through deployment of a technology already developed elsewhere, but still requiring technology development work.

Low: One STCG-submitted need and one other potential benefiting site based on Technology Integration Manager (TM) judgment.

User Commitment - The TFA values user commitment to the development and deployment of technical solutions. This criterion assesses the strength of user commitment to share the burden of a technology's development and deployment.

High:

- Site co-funds development and demonstration (or deployment)

- High commitment to deploy through out-year baseline, PBS, and budget request; memorandum of understanding (MOU) or other signed document for TFA next year expenditures over $\$ 1 \mathrm{M}$

- Currently in site baseline operational plan with MOU or other signed document committing to funding and plan for deployment in subject FY

- Deployment within $1-2$ years

- Greater than or equal to 50/50 co-funding of development and demonstration for the year of prioritization and duration of the response.

High/Medium: Response results in data delivery for key DOE decisions, e.g., Environmental Impact Statement (EIS) or privatization decisions.

- Site co-funds data development and delivery

- Data will be used within $1-2$ years 
- High commitment to deploy through out-year baseline, PBS, and budget request; MOU or other signed document for TFA expenditures over $\$ 1 \mathrm{M}$

- Greater than or equal to 50/50 co-funding of development and delivery for the year of prioritization and duration of the technical response.

Medium/High: Approximately equal co-funding to develop and demonstrate during time of the technical response. High commitment to deploy through out-year baseline, PBS, and budget request; TFA Site Representative commitment to obtain MOU or other signed document for TFA next year expenditures over $\$ 1 \mathrm{M}$.

Medium: Approximately one-quarter co-funding; high commitment to deploy through out-year baseline, PBS, and budget request; TFA Site Representative commitment to obtain MOU or other signed document for TFA next year expenditures over $\$ 1 \mathrm{M}$.

Low: Site co-funding exists, but no commitment to deploy or use data (e.g., not in sites' out-year planning documents).

Note on co-funding: Co-funding is to be focused on support to the overall project the TFA is funding. This may include direct support to PI, support to on-site operations staff to facilitate testing, sample collection/analysis/shipping, design and review. Also, the TFA Management Team may require a MOU or some other documented user commitment on any task under consideration for TFA funding.

Technical Risk - This criterion considers technical risks related to site baselines.

\section{Needs Priority}

- High: Technical response addresses at least two needs with a priority of 1 , or three needs with a priority of 2 .

- Medium: Technical response addresses at least one need with a priority of 1 , or two needs with a priority of 2 .

- Low: Technical response addresses at least one need with a priority of 2. (Note: no value is assigned to a technical response addressing needs with a priority of 3.)

\section{Technical Risk}

- High: Related waste stream technical risk is high (risk rating of 4 or 5), or related critical path milestone technical risk is high (risk rating of 4 or 5 )

- Medium: Related waste stream technical risk medium with a risk rating of 3, or related critical path milestone technical risk is medium (risk rating of 3 ) 
- Low: Related waste stream technology risk is medium or low with a risk rating of 2 or 1 , or related critical path milestone technical risk is medium or low (risk rating of 2 or 1 ).

Other Technology Impact - The objective of this criterion is to broadly assess the overall potential technology impact of a technical response. The TFA considers a response's impact on schedule, cost avoidance, and link to regulatory requirements to determine impact. The ratings include the following:

High: (one or more of the following apply)

- Technology required to meet baseline assumptions

- Documented high cost avoidance (over $\$ 250 \mathrm{M}$ ) to EM (information must be provided to TFA by site with uncertainty analysis)

- Possesses high cost reduction potential (over $\$ 250 \mathrm{M}$ )

- Required to meet firm regulatory requirements that could delay tank waste remediation schedules.

Medium: (one or more of the following apply)

- Required to meet enhancements or alternatives to baselines

- Documented moderate cost avoidance (between $\$ 250 \mathrm{M}$ and $\$ 50 \mathrm{M}$ ) to EM or general consensus on high cost avoidance (over $\$ 250 \mathrm{M}$ ) that cannot be documented due to lack of data that will be developed if the task goes forward

- Possesses moderate cost reduction potential

- Adds assurance that regulatory requirements are met, or supports a regulatory requirement that the site may renegotiate.

Low: (one or more of the following)

- Appears that technology could meet baseline or enhancement assumptions, but more data is needed and will be provided explicitly if the task proceeds

- General consensus that moderate cost avoidance (between $\$ 250 \mathrm{M}$ and $\$ 50 \mathrm{M}$ ) could be achieved but cannot yet be documented

- The technical response's link to regulatory requirements is not fully determined. 
In March 2000, the TFA evaluated each technical response using the approved criteria. This initial assessment was accomplished in a group consensus of TIMs, monitored by the TFA Program Management Team. The TFA's intent was to ensure that technical responses would

- Be provided for each need received

- Contain an explanation of the priority of the response according to either

- Screening criteria

- Prioritization criteria

- Describe multiyear intent

- 4-year budget estimate (current +3 years)

- Basis of estimate

- Describe the intended scope

- Identify the relationship or benefit to other site needs.

\subsection{TFA Management Team Prioritization}

The TFA technical response prioritization took place on March 9, 2000 in conjunction with TFA Midyear activities. During prioritization, the TFA Management Team assigned final scores to each technical response against the approved criteria. The Management Team discussed the merits of the responses, focusing closely on aspects of site benefits, user commitment, and continuity of ongoing technology development. Additionally, the Management Team reviewed and approved six strategic tasks for inclusion into the FY 20012002 program. At the conclusion of the prioritization session, the Management Team affirmed the results, thereby creating the official TFA FY 2001-2002 Integrated Priority Listing (IPL).

As of the publication date of this document, the TFA is finalizing the technical responses to incorporate actions directed by the Management Team during prioritization. The final version of the technical responses will be posted on the Technical Team home page (http://www.pnl.gov/tfa/program) in the near future.

\subsection{Data Summary}

In all, the TFA received 140 science and technology needs. The TFA assigned each need to one of the TFA's six functional areas based on the major subject area of the need. Some needs statements were broad enough that they required action in more than one technical response. In all, 63 technical responses were prepared by the TFA. A summary of the TFA's functional assignment of needs and technical responses by site is shown in Table 2.2. 
Table 2.2. Summary of Site Needs Submitted to the Tanks Focus Area

\begin{tabular}{|l|c|c|c|c|c|c|}
\hline & Hanford & INEEL & ORR & SRS & WVDP & Total \\
\hline Safety & 4 & 2 & 1 & 2 & 1 & 10 \\
\hline Characterization & 3 & 8 & 1 & 1 & 1 & 14 \\
\hline Pretreatment & 2 & 13 & 2 & 4 & 0 & 21 \\
\hline Immobilization & 4 & 11 & 1 & 4 & 2 & 22 \\
\hline Retrieval & 8 & 1 & 2 & 6 & 2 & 19 \\
\hline Closure & 4 & 6 & 1 & 1 & 1 & 13 \\
\hline Total & 56 & 45 & 7 & 24 & 8 & 140 \\
\hline
\end{tabular}

The needs across the complex reflect requirements to

- Inspect tank integrity, monitor tank corrosion, and detect and mitigate leaks

- Reduce waste volumes and minimize the generation of additional wastes, including secondary wastes

- Sample and characterize the chemical and physical properties of the wastes

- Retrieve salt and sludge wastes and tank heels

- Pump and transfer wastes efficiently without plugging pipelines

- Separate radionuclides from non-radioactive waste components

- Provide grout and glass waste forms for LLW immobilization and disposal

- Optimize waste loadings in HLW glass waste forms

- Enhance design of HLW glass melters

- Improve efficiency of existing waste storage and treatment facilities operations and maintenance

- Access waste residuals as precursors to additional retrieval and tank closure decisions

- Immobilize waste residuals and stabilize tanks as part of closure.

Hanford and SRS require continued emphasis on determining the impacts of waste chemistry on waste retrieval and transport. Hanford and SRS require additional mixing technologies to suspend sludges and saltcake for waste removal. SRS requires technical data to support the selection, design, and implementation of an alternative to the in-tank precipitation process for radionuclide removal. As waste storage and processing facilities mature, technologies are needed for remote maintenance and repair and to optimize equipment design for improved operations. INEEL needs technical data to support process selection and design for liquid and calcine wastes. WVDP and SRS require improved technologies for HLW canister decontamination. Hanford needs additional data and tools to support waste disposal system performance assessments.

During its analysis of the site needs, the TFA found that many of the requirements from any one site have multi-site benefit. The TFA will exploit the resolution of these requirements to leverage these multi-site benefits. Multi-site benefit is one of the four criteria the TFA used this year in prioritizing future work. The tentative program for FY 2001 - FY 2002 reflects the importance the TFA places on multi-site benefit. 


\subsection{Lessons Learned}

Every year, the TFA learns new lessons in executing the initial stages of the program development process. Last year was the first full program development cycle where the TFA went into the process expecting to perform in accordance with the "Focus Area-centered" concept. The Focus Area-centered concept calls for Focus Areas, such as the TFA, to lead the coordination and integration of all OST programs within its problem area. Coordinating site needs analyses and technical response development with and between the TFA's partner programs continues to be a sizeable task, and the TFA appreciates the efforts of its partner programs to help meet the challenge. To increase the efficiency of the coordination and integration, the TFA is negotiating operating agreements between it and the TFA's three main Crosscutting program partners (CMST, ESP, and Robotics). Memorandums of Understanding (MOUs) between the TFA and each of these three programs are presently being finalized.

The requirement exists to better synchronize, at the EM and OST level, the scheduling of program development activities that culminate in the TFA's preparation and submission of draft budget documents in the March-April timeframe of each year. Development and use of the Integrated Planning, Accounting, and Budgeting System (IPABS) continues to be a serious impediment to program development. More work is required during the next year to make the system less cumbersome, more reliable, and better integrated. Data quality issues remain, especially in waste stream linkages to site needs and technical risks existing in those waste streams. The TFA has taken on an added burden of helping identify to the sites where data inconsistencies exist. Although this is the second year that the IPABS system is being used to develop OST work package priorities, there continues to be great uncertainty about data quality, rating criteria, and system scheduling requirements leading up to work package prioritization.

The TFA continues to grow into its perceived Focus Area-centered responsibilities in the basic science portion of its investment portfolio. While some progress was made during the last year in strengthening relationships with the Environmental Science Management Program (EMSP) staff, serious program development and execution issues still require significant attention. 


\section{Section 3 - The Next Process Steps}

As noted earlier, this document reports only on the initial program development steps. Formulation of the final detailed technical response for each submitted need is in progress. The first phase of the FY 2002 Corporate Review Budget (CRB) development is completed. The purpose of this section is to describe how the activities covered in this site needs assessment fit into the overall program development process and to provide a short description of the remaining program development activities. Within the overall program development process, the following major tasks and schedule remain for this year's program development cycle:

- Finalize FY 2002 CRB submittal (May 2000)

- OST work package prioritization for the FY 2002 CRB (May 2000)

- Prepare and submit FY 2001 program execution documents (June-August 2000)

- Prepare Multiyear Program Plan (MYPP) (September 2000)

- High-Level Waste Steering Committee (HLWSC) approval of MYPP (October 2000).

\subsection{Finalize FY 2002 CRB Submittal}

The TFA is completing preparation of its FY 2002 CRB budget input based on the prioritized technical responses to site needs. The TFA groups technical responses by functional subject area and TFA priority into "work packages." Work packages are the main components of the TFA's CRB.

\subsection{OST Work Package Prioritization for the FY 2002 CRB}

OST rates each focus area work package according to pre-established criteria. Presently, these criteria value

- PBSs - the number of them represented, their life cycle costs, and significant milestones

- Likelihood of technology deployments

- Priority of site needs addressed

- Technical risk

- Potential cost savings.

The result of the rating is a prioritized list of work packages for DOE management consideration within expected available funding. The TFA supports the prioritization activity by ensuring, through coordination with its user sites, the most accurate data is available. 


\subsection{Prepare and Submit FY 2001 Program Execution Documents}

Each year, the TFA uses two documents to provide for program execution. The first, the Program Execution Guidance (PEG), is the TFA's guidance to the selected work performers and is tied to the users' commitment and priority. This guidance states the mandatory technical and programmatic requirements needed for each task. The PEG is simply an expansion of the final technical responses that have been reviewed and approved by the TFA Management Team.

Upon receipt of the PEG, the performer develops the second document, the Technical Task Plan (TTP). The TTP is the performer's response to the PEG. An approved TTP constitutes a contractual arrangement between the TFA, the performing DOE Field Office, and the performing organization. Both documents are generally required before work initiation and funding authorization.

During the transition between PEG and TTP, the TFA coordinates with sites and performers to assure site commitment to each technical response, that all performer selection issues have been resolved, and that the proposed scope and budget are understood fully by all.

\subsection{Document in the MYPP}

The companion document to this one is the TFA MYPP. The MYPP documents the results of the preceding planning steps and is the basis for complementary planning between OST and the Offices of Environmental Restoration and Waste Management in future years, which is reflected in the OST budget process. This approach is consistent with the TFA goal of defining and implementing an integrated technical program. The MYPP describes the TFA's technical strategies and the actions being taken to address the site needs within the strategies. The FY 2001 - FY 2005 MYPP is expected to be published during September 2000.

Each year, the MYPP is updated to reflect the changing emphasis of the sites and the subsequent changes in the TFA's technical focus. Based on the FY 2001 site needs submittal and the resulting technical responses, the FY 2001 - FY 2005 MYPP should show the TFA's continuing emphasis to

- Provide technologies that support waste retrieval and tank closure at SRS, Hanford, INEEL, WVDP, and ORR

- Appropriately support DOE's privatization of tank waste management activities at Hanford and ORR

- Provide technical answers to vitrification requirements from around the complex

- Support development and implementation of the alternative to in-tank precipitation at SRS

- Support INEEL in process selection and design

- Provide technologies for monitoring tank integrity and corrosion. 
Tanks Focus Area (TFA). 1999a. Tanks Focus Area Site Needs Assessment FY 1999, PNNL-12187. Pacific Northwest National Laboratory, Richland, Washington.

Tanks Focus Area (TFA). 1999b. Tanks Focus Area Multiyear Program Plan FY00-FY04, PNNL-12264. Pacific Northwest National Laboratory, Richland, Washington. 


\section{Appendix A - Site Needs Database}

This appendix summarizes the 140 science and technology needs submitted by the sites and the TFA's intended technical disposition of them. Table A.1 is a list of the needs received from each site and identification of the technical response or responses linked to that need. Table A.2 takes those same needs and aligns them within the TFA problem element structure. Additionally, Table A.2 lists the need priority assigned by the site to the need, and the functional area the TFA assigned to the need. Note that a need may occur more than once in the problem element structure. This is because a need may be broad enough that it is described best in more than one problem element, and therefore will likely appear in more than one technical response.

Table A.3 portrays the TFA's interpretation of the benefiting sites for each technical response. The technical responses are listed in the priority order established by the TFA Management Team.

The remainder of the appendix is devoted to the individual site needs. This document only provides a summary of each need. Interested readers may find full versions of the site highlevel tank waste needs at the following web sites:

- Hanford: http://www.pnl.gov/stcg/needs.stm

- INEEL: http://stcgneeds.inel.gov/wt select.asp?id=HLW

- ORR: http://www.em.doe.gov/usrbin/techneed/qu/sg?stcg=TANKS\&site=OAK+RIDGE+NATIONAL+LABORATORY\& category=Any\&contam=Any

- SRS: $\underline{\text { http://www.srs.gov/general/srtech/stcg/needstmt.htm }}$

- WVDP: http://www.ohio.doe.gov/oh-stcg/needs.asp

Additionally, a comprehensive library of science and technology needs is found in EM's Needs Management System at: http://em-needs.em.doe.gov.

This appendix provides a brief summary of each site needs statement. The summaries were largely extracted from the actual needs statements found in the above web sites. Following the summary for each need is the number, title, and FY 2001 - FY 2002 TFA priority number for the technical response to that need. In several cases, the TFA responded to individual needs in more than one technical response.

- Hanford needs begin on page A.14

- INEEL needs begin on page A.36

- ORR needs begin on page A.58

- SRS needs begin on page A.60

- WVDP needs begin on page A.71. 
Table A.1. Tanks Focus Area Needs Submitted by Sites

\begin{tabular}{|c|c|c|}
\hline \multirow{2}{*}{$\begin{array}{l}\text { Site Need \# } \\
\text { HANFORD }\end{array}$} & Need Title & TFA Response \# \\
\hline & & \\
\hline RL-WTOT & $\begin{array}{l}\text { Technetium-99 Analysis in Hanford Tank Waste and Contaminated Tank } \\
\text { Farm Areas }\end{array}$ & A9264 \\
\hline RL-WTO4 & Double-Shell Tank Corrosion Monitoring & A9143 \\
\hline RL-WTO5 & Remote Inspection of High-Level Waste Single-Shell Tanks & $\begin{array}{l}\text { A9175, AAISI, } \\
\text { AA7S1 }\end{array}$ \\
\hline RL-WT09 & $\begin{array}{l}\text { Representative Sampling and Associated Analysis to Support Operations } \\
\text { and Disposal }\end{array}$ & A9246 - \\
\hline RL-WT013 & Establish Retrieval Performance Evaluation Criteria & $\begin{array}{l}\text { A9367, A9947, } \\
\text { AA203, AA1S1, } \\
\text { AA3S2 }\end{array}$ \\
\hline RL-WT015 & Standard Method for Determining Waste Form Release Rate & A9748 \\
\hline RL-WT016 & Glass Monolith Surface Area & A9749 \\
\hline RL-WTO17 & Long-Term Testing of Surface Barrier & A9950 \\
\hline RL-WTO18 & Testing of Sand-Gravel Capillary Barrier & A9950 \\
\hline RL-WT021 & Cleaning, Decontaminating and Upgrading Hanford Pits & A9352, AAIS1 \\
\hline RL-WT022 & Tank Knuckle NDE & A9175, AA1S1 \\
\hline RL-WTO23 & $\begin{array}{l}\text { Prediction of Solid Phase Formation in Static and Dynamic Hanford Tank } \\
\text { Waste Solutions }\end{array}$ & $\begin{array}{l}\text { A9367, A9554, } \\
\text { AA3S1 }\end{array}$ \\
\hline RL-WT024 & Enhanced Sludge Washing Process Data & $\begin{array}{l}\text { A9555, AA3S1, } \\
\text { AA5S1 }\end{array}$ \\
\hline RL-WT026 & $\begin{array}{l}\text { Tank Leak Detection Systems for Underground Single-Shell Waste } \\
\text { Storage Tanks (SSTs) }\end{array}$ & A9156 \\
\hline RL-WT027 & Tank Leak Mitigation Systems & $\begin{array}{l}\text { A9157, AA1S1, } \\
\text { AA3S2 }\end{array}$ \\
\hline RL-WT029 & Data and Tools for Performance Assessments & A9958 \\
\hline RL-WT060 & Better Waste Mixing Mobilization & A9359, AA3ST \\
\hline RL-WT061 & Reactive Barriers to Contaminant Migration & A9960 \\
\hline RL-WT062 & Variable Suction Level Transfer Pump & A9365 \\
\hline RL-WT063 & $\begin{array}{l}\text { PHMC Retrieval and Closure - Hanford SST Saltcake Dissolution } \\
\text { Retrieval }\end{array}$ & $\begin{array}{l}\text { A9362, A9554, } \\
\text { AA3S1, AA3S2 }\end{array}$ \\
\hline RL-WT064 & $\begin{array}{l}\text { PHMC Retrieval and Closure - Hanford Past Practice Sluicing } \\
\text { Improvements }\end{array}$ & $\begin{array}{l}\text { A9367, AA3S1, } \\
\text { AA3S2 }\end{array}$ \\
\hline RL-WT065 & Direct Inorganic and Organic Analyses of High-Level Waste & A9264 \\
\hline RL-WT066 & $\begin{array}{l}\text { Compositional Dependence of the Long Term Performance of Glass as a } \\
\text { Low-Activity Waste Form }\end{array}$ & A9748, AA7S1 \\
\hline RL-WT067 & Improved DST Integrity NDE Measurement Tools & A9175, AA1S1 \\
\hline RL-WT068 & Radionuclide Source Term from Tank Residuals & A9588 \\
\hline RL-WT069 & Value of Information Decision Analysis for Tank Farm Closure & A9924 \\
\hline RL-WT070 & $\begin{array}{l}\text { Uncertainty Estimation of Hanford Best Basis Toxic Waste lnventory, } \\
\text { Concentration, Phase and Waste Type }\end{array}$ & $\begin{array}{l}\text { A9555, AA3ST, } \\
\text { AA5S1 }\end{array}$ \\
\hline RL-WT071 & $\begin{array}{l}\text { Provide Laboratory Development Support and ESP Modeling Support for } \\
\text { the Back Dilution of Tank 241-SY-101 }\end{array}$ & A9554, AA3S1 \\
\hline RL-WT072 & $\begin{array}{l}\text { Use of Handheld Technology to Automate Operator Data Sheets for Tank } \\
\text { Farm Operations }\end{array}$ & AAIOT \\
\hline RL-WT080 & Advanced/Improved Vitrification & $\begin{array}{l}\text { A9768, A9773, } \\
\text { AA7S1, AA7S2 }\end{array}$ \\
\hline RL-WT081 & Sulfate Accumulation in Low Activity Waste & A9773, AA7S1 \\
\hline RL-WT082 & Crystalline Silicotitanate Non-Elutable Sorbent & A9570 \\
\hline RL-WT083 & Rapid PCB Screening Technology & A9264 \\
\hline RL-WT084 & Extension of Glass Properties Model to Lower Silica Compositions & A9773 \\
\hline
\end{tabular}




\begin{tabular}{|c|c|c|}
\hline Site Need \# & Need Title & TFA Response \# \\
\hline RL-WT085 & $\begin{array}{l}\text { Retrieval of Waste Heel from } 340 \text { Radioactive Liquid (Low-Level/Mixed } \\
\text { Waste Vault) Vault Tanks }\end{array}$ & A9382 \\
\hline RI-WTO31-S & Rapid Waste Characterization & AA202 \\
\hline RL-WTO32-S & $\begin{array}{l}\text { Monitoring of Key Waste Physical Properties During Retrieval and } \\
\text { Transport }\end{array}$ & A9278 \\
\hline RL-WT035-S & Moisture Flow and Contaminant Transport in Arid Conditions & A9958 \\
\hline RL-WT037-S & Sludge Treatment & $\begin{array}{l}\text { A9555, AA3S1, } \\
\text { AA5S1. }\end{array}$ \\
\hline RL-WTO38-S & Process Models for Sludge Treatment & $\begin{array}{l}\text { A9555, AA3S1, } \\
\text { AA5S1 }\end{array}$ \\
\hline RL-WTO40-S & Mechanisms of Line Plugging & $\begin{array}{l}\text { A9376, A9554, } \\
\text { AA3S1 }\end{array}$ \\
\hline RL-WT041-S & Radionuclide Partitioning & None \\
\hline RL-WT043-S & $\begin{array}{l}\text { Effect of Human and Natural lnfluences on Long-Term Water } \\
\text { Distribution }\end{array}$ & A9958 \\
\hline RL-WT044-S & Distribution of Recharge Rates & A9958 \\
\hline RL-WT045-S & Vadose Zone Flow Simulation Tool Under Arid Conditions & A9958 \\
\hline RL-WTO46-S & Getter Materials & A9960 \\
\hline RL-WT049-S & $\begin{array}{l}\text { Effect of Processing on Gas Release, Waste Sedimentation, Rheological, } \\
\text { and Other Behaviors }\end{array}$ & A9554, AA3S1 \\
\hline RL-WT052-S & $\begin{array}{l}\text { Characterization of Organic Species in Waste Feed to LAW and HLW } \\
\text { Treatment Facilities }\end{array}$ & A9264 \\
\hline RL-WT053-S & Contaminant Mobility Beneath Tank Farms & None \\
\hline RL-WTO54-S & Solids Yield During Mixer Pump Mobilization & A9359 \\
\hline RL-WT056-S & Half-Lives of Se-79 and Sn-126 & A9958 \\
\hline RL-WTO75-S & HLW Solid Phase Characterization & A9554, AA3SI \\
\hline RL-WT076-S & Plutonium Interaction with Silicates & None \\
\hline RL-WTOT7-S & Improvements to Salt Well Pumping & A9362, AA3S2 \\
\hline RL-WTO78-S & Plutonium Segregation and Association in HLW & $\begin{array}{l}\text { A9555, AA3S1, } \\
\text { AA5S1 }\end{array}$ \\
\hline RL-WT079-S & Double Shell Tanks Corrosion Chemistry & A9143 \\
\hline INEEL & & \\
\hline $\mathrm{ID}-2.1 .06$ & TRU, Cs and $\mathrm{Sr}$ Removal from High Activity Wastes & A9501 \\
\hline ID-2.1.15 & Neutralization of Newly Generated Liquid Wastes & A9502 \\
\hline $\mathrm{D}-2.1 .16$ & Decontamination Facility/Analytical Facility Waste Reduction & A9264, A9508 \\
\hline $\mathrm{ID}-2.1 .17$ & Develop New Filter Leach Process & A9508 \\
\hline $\mathrm{DD}-2.1 .19$ & EPA Methods Sample Collection and Analysis Verification/Development & A9206 \\
\hline $1 \mathrm{D}-2.1 .20$ & Tank Annulus/ $V$ ault Inspection & A9175, AA1S1 \\
\hline ID-2.1.23 & Low-Activity Wasteform Qualification & A9719 \\
\hline ID-2.1.24 & $\begin{array}{l}\text { Tntegration/Optimization of High Activity Waste/Low Activity Waste } \\
\text { Process Flowsheet }\end{array}$ & A9709 \\
\hline $\mathrm{TD}-2.1 .25$ & Ion-Exchange System for Water Runoff & A9510 \\
\hline $\mathrm{ID}-2.1 .26$ & Direct Tank Sampler for Tank Solution Characterization & A9246 \\
\hline $\mathrm{ID}-2.1 .27$ & Blowback Metal Filters for Solids (Calcine) Retrieval & A9171 \\
\hline $\mathrm{ID}-2.1 .28$ & Cs and Sr Removal from Newly Generated Liquid Waste & A9570, A9719 \\
\hline $\mathrm{ID}-2.1 .29$ & Evaluate Chloride Corrosion Potential (LET\&D/PEWE/Future Processes) & A9514 \\
\hline $\mathrm{ID}-2.1 .30$ & Remove/Treat Chlorides (LET\&D/PEWE/Future Processes) & A9514 \\
\hline $\mathrm{ID}-2.1 .31$ & Characterization of Entrainable Solids in Tank Waste & A9216 \\
\hline $\mathrm{ID}-2.1 .35$ & $\begin{array}{l}\text { Direct Immobilization of INTEC Sodium-Bearing and Newly Generated } \\
\text { Liquid Wastes }\end{array}$ & A9719 \\
\hline $\mathrm{ID}-2.1 .36$ & Mercury Removal from Liquid Wastes & A9518 \\
\hline
\end{tabular}


Table A.1. Continued

\begin{tabular}{|c|c|c|}
\hline Site Need \# & Need Title & TFA Response \# \\
\hline ID-2.1.38 & Conditioning of Low Activity Waste for Treatment & A9719 \\
\hline ID-2.1.39 & Acceptance Criteria for LAW Disposal in Underground Storage Tanks & A9924 \\
\hline ID-2.1.40 & Low Activity Waste Grout Sorbent Addition to Reduce Leachability & A9719 \\
\hline ID-2.1.41 & HLW Process Offgas Treatment & A9722 \\
\hline $\mathrm{ID}-2.1 .42$ & Acceptance Criteria for Tank Closure & A9924 \\
\hline ID-2.1.43 & $\begin{array}{l}\text { Certify LDUA Sampler as EPA-Approved Method of Sampling Tank } \\
\text { Heel Liquids }\end{array}$ & A9246 \\
\hline ID-2.1.44 & $\begin{array}{l}\text { Certify LDUA Sampler as EPA-Approved Method of Sampling Tank } \\
\text { Heel Solids }\end{array}$ & A9246 \\
\hline $\mathrm{TD}-2.1 .45$ & Acceptance Criteria for Grouting Tank Heels & A9924 \\
\hline $\mathrm{ID}-2.1 .46$ & Management of Tank Heel Liquids & A9923 \\
\hline ID-2.1.47 & Management of Tank Heel Solids & A9923 \\
\hline $\mathrm{ID}-2.1 .48$ & $\begin{array}{l}\text { Wasteform Qualification for Low-Activity Waste in Underground Storage } \\
\text { Tanks }\end{array}$ & A9924 \\
\hline ID-2.1.49 & Acceptance Criteria for High Activity Waste/Low Activity Waste & A9730 \\
\hline ID-2.1.50 & Solids Waste (Calcine) Retrieval & A9331 \\
\hline ID-2.1.51 & Develop Calcine Dissolution Kinetics for Solid/Liquid Equilibria & A9532 \\
\hline $\mathrm{ID}-2.1 .52$ & Characterization of Solids from Calcine Dissolution & A9532 \\
\hline ID-2.1.56 & Mercury Treatment for Aluminum Calcine & A9501, A9518 \\
\hline ID-2.1.57 & Conditioning of HAW for Treatment & A9768, AA7S2 \\
\hline $\mathrm{TD}-2.1 .58$ & HAW Immobilization & $\begin{array}{l}\text { A9768, A9773, } \\
\text { AA7S1, AA7S2 }\end{array}$ \\
\hline ID-2.1.62 & Acceptance Criteria for Bin Set Closure & A9924 \\
\hline ID-2.1.64 & Solid-Liquid Separation Equipment Development and Application & A9584 \\
\hline ID-2.1.65 & Treatment/Disposition of Removed Tank Solids & A9709 \\
\hline $\mathrm{ID}-2.1 .66$ & Treatment/Disposition of Spent lon Exchange Resins & $\begin{array}{l}\text { A9719, A9768, } \\
\text { A9773, AA7S1 }\end{array}$ \\
\hline ID-2.1.67 & High Level Waste Slurry Handling & $\begin{array}{l}\text { A9278, A9361, } \\
\text { A9365, AA7S2 }\end{array}$ \\
\hline IID-2.1.68 & Technetium Removal from INEEL High Level Waste & A9501 \\
\hline $110-2.1 .69$ & Solids Waste (Calcine) Retrieval from CSSF1 & A933I \\
\hline $\mathrm{D}-2.1 .70$ & Low-Activity Waste Biodegradation Test & AA902 \\
\hline $\mathrm{ID}-2.1 .71$ & Grout/Heel Mix in Place System & A9985 \\
\hline $\mathrm{TD}-2.1 .72$ & Alternate Heel Sampling Systems & AA203, AA1SI \\
\hline \multicolumn{3}{|l|}{ ORR } \\
\hline ORTK-01 & Tank Waste Characterization & $\begin{array}{l}\text { A9143, A9175, } \\
\text { AA1S1 }\end{array}$ \\
\hline ORTK-02 & Tank Solid Waste Retieval & $\begin{array}{l}\text { A9359, A9367, } \\
\text { A9382, AA3S2 }\end{array}$ \\
\hline ORTK-04 & Sludge Mixing and Slurry Transport & $\begin{array}{l}\text { A9278, A9554, } \\
\text { AA3S1 }\end{array}$ \\
\hline ORTK-05 & Tank Sludge and Supernatant Separations & $\begin{array}{l}\text { A9555, A9586, } \\
\text { AA3S1, AA5S1 }\end{array}$ \\
\hline ORTK-06 & Tank Sludge Supernatant Immobilization & A9719 \\
\hline ORTK-09 & Tank Closure & A9923, A9985 \\
\hline ORTK-11 & Tank Supernatant Pretreatment & A9570, A9586 \\
\hline \multicolumn{3}{|l|}{ SRS } \\
\hline SRO0-1011 & $\begin{array}{l}\text { Demonstrate Evaporation Technologies to Reduce Generation of } \\
\text { Secondary Waste Volume from Consolidated Incineration Facility }\end{array}$ & A9586 \\
\hline SR00-2027 & Demonstrate Alternative Filtration Technologies to Replace HEPA Filters & A9171 \\
\hline
\end{tabular}


Table A.1. Continued

\begin{tabular}{|c|c|c|}
\hline Site Need \# & Need Title & TFA Response \# \\
\hline SR00-2028 & Alternative Waste Removal Technology & $\begin{array}{l}\text { A9359, A9362, } \\
\text { AA3S1, AA3S2 }\end{array}$ \\
\hline SR00-2029 & Alternate DWPF Canister Decon Technology & A9772 \\
\hline SR00-2031 & Develop Remote Technology to Improve DWPF Operations & A9374 \\
\hline SRO0-2032 & Optimize Melter Glass Chemistry and lncrease Waste Loading & $\begin{array}{l}\text { A9773, AA7S1, } \\
\text { AA7S2 }\end{array}$ \\
\hline SR00-2033 & $\begin{array}{l}\text { Provide Alternative Processing and/or Concentration Methods for DWPF } \\
\text { Recycle Aqueous Streams }\end{array}$ & A9566 \\
\hline SRO0-2034 & Second Generation Salt Feed Preparation & A9570 \\
\hline SR00-2035 & $\begin{array}{l}\text { Develop Advanced Techniques for Life Extension of High Level Waste } \\
\text { Tanks and Piping }\end{array}$ & A9175, AAIS1 \\
\hline SR00-2036 & Develop Improved HLW Melter & $\begin{array}{l}\text { A9768, A9773, } \\
\text { AA7S1, AA7S2 }\end{array}$ \\
\hline SR00-2037 & Tank Hee Removal/Closure Technology & $\begin{array}{l}\text { A9157, A9175, } \\
\text { A9278, A9352, } \\
\text { A9359, A9363, } \\
\text { A9365, A9367, } \\
\text { A9382, A9554, } \\
\text { AA203, AA204, } \\
\text { AA303, AA1S1, } \\
\text { AA3Si, AA3S2 }\end{array}$ \\
\hline SR00-2039 & Methods to Unplug Waste Transfer Lines & $\begin{array}{l}\text { A9376, A9554, } \\
\text { AA3S1 }\end{array}$ \\
\hline SR00-2040 & $\begin{array}{l}\text { Demonstrate Remote Decommissioning and Disassembly of High Level } \\
\text { Waste Processing Equipment }\end{array}$ & A9777 \\
\hline SR00-2041 & Develop Advanced Mixing Technology & A9359 \\
\hline SR00-2044 & In-Situ Technology for Waste Characterization & $\begin{array}{l}\text { A9278, AA201, } \\
\text { AA202 }\end{array}$ \\
\hline SR00-2045 & In-Situ Waste Tank Corrosion Probe & A9143 \\
\hline SR00-2051 & $\begin{array}{l}\text { Technology to Mitigate Effects of Technetium Under Tank Closure } \\
\text { Conditions }\end{array}$ & A9588, A9960 \\
\hline SR00-2052 & $\begin{array}{l}\text { Aluminum Dissolution from HAW Sludge and its Impact on Downstream } \\
\text { Salt Processing }\end{array}$ & $\begin{array}{l}\text { A9555, AA3S1, } \\
\text { AA5S1 }\end{array}$ \\
\hline SR00-2055 & Increase in Applicability/Efficiency of High-Level Waste Planning Tool & A9709 \\
\hline SR00-3022 & $\begin{array}{l}\text { In-Situ Grouting and/or Retrieval of Waste from Underground Tanks } \\
\text { (Formerly Used for the Storage of Radioactive Solvents) }\end{array}$ & A9923, A9985 \\
\hline SR00-2049-S & Technetium Chemistry Under Waste Removal Conditions & A9588 \\
\hline SR00-2050-S & $\begin{array}{l}\text { Fracture Toughness Properties for Carbon Steel Utilized for Nuclear } \\
\text { Waste Containment Vessels }\end{array}$ & A9175, AAIS1 \\
\hline SR00-2053-S & $\begin{array}{l}\text { Develop an Alternative Sorbent to Replace Monosodium Titanate for } \mathrm{Sr} \\
\text { and Actinide Removal }\end{array}$ & A9570 \\
\hline SR00-2054-S & $\begin{array}{l}\text { Develop Improved Radiochemical Analysis for High lonic Strength } \\
\text { Samples }\end{array}$ & A9264 \\
\hline \multicolumn{3}{|l|}{ WVDP } \\
\hline OH-WV-902 & Decontamination of High-Level Waste (HLW) Canisters (WVDP-2-99) & A9772 \\
\hline OH-WV-903 & Vitrification Expended Material Processing (WVDP-3-99) & A9777 \\
\hline OH-WV-904 & High Level Waste Tank Closure & A9950, AA310 \\
\hline OH-WV-905 & Retrieval of Tank Heels & A9361, A9382 \\
\hline OH-WV-906 & Radioactivity Measurement of High-Level Waste Tank Residuals & AA202 \\
\hline OH-WV-907 & High-Level Waste Tank Interim Maintenance & A9175, AA1S1 \\
\hline OH-WV-908 & Decontamination of High-Level Waste Contaminated Equipment & AA311 \\
\hline OH-WV-914 & Development of Grout for In-Situ Closure & A9923 \\
\hline
\end{tabular}


Table A.2. Tanks Focus Area Site Needs Distributed within the Problem Element Structure

\begin{tabular}{|c|c|c|c|c|}
\hline & & & Need & \\
\hline PE\# & Problem Element Title & Site & $\underline{\text { Pri }}$ & Function \\
\hline 1.1 & Store Waste & & & \\
\hline 1.1.1 & Extend Tank Life & & & \\
\hline 1.1.1.1 & Monitor Tank Integrity/Avoid Corrosion & & & \\
\hline RL-WT04 & Double-Shell Tank Corrosion Monitoring & Hanford & 1 & Safety \\
\hline RL-WT05 & $\begin{array}{l}\text { Remote Inspection of High-Level Waste } \\
\text { Single-Shell Tanks }\end{array}$ & Hanford & 2 & Safety \\
\hline RL-WT022 & Tank Knuckle NDE & Hanford & 2 & Safety \\
\hline RL-WT067 & Improved DST Integrity NDE Measurement Tools & Hanford & 2 & Safety \\
\hline RL-WT072 & $\begin{array}{l}\text { Use of Handheld Technology To Automate } \\
\text { Operator Data Sheets For Tank Farm Operations }\end{array}$ & Hanford & 2 & Safety \\
\hline RL-WT079-S & Double Shell Tanks Corrosion Chemistry & Hanford & 1 & Safety \\
\hline $\mathrm{ID}-2.1 .20$ & Tank Annulus/Vault Inspection & INEEL & 1 & Safety \\
\hline ORTK-01 & Tank Waste Characterization & ORR & 3 & Safety \\
\hline SR00-2035 & $\begin{array}{l}\text { Develop Advanced Techniques for Life Extension } \\
\text { of High Level Waste Tanks and Piping }\end{array}$ & SRS & 3 & Safety \\
\hline SR00-2037 & Tank Heel Removal/Closure Technology & SRS & 1 & Safety \\
\hline SR00-2045 & In-Situ Waste Tank Corrosion Probe & SRS & 2 & Safety \\
\hline SR00-2050-S & $\begin{array}{l}\text { Fracture Toughness Properties for Carbon Steel } \\
\text { Utilized for Nuclear Waste Containment Vessels }\end{array}$ & SRS & 1 & Safety \\
\hline OH-WV-907 & High-Level Waste Tank Interim Maintenance & WVDP & 2 & Safety \\
\hline 1.1.3 & Characterize Waste & & & \\
\hline 1.1 .2 & Ventilate Tanks & & & \\
\hline $\mathrm{ID}-2.1 .27$ & $\begin{array}{l}\text { Blowback Metal Filters for Solids (Calcine) } \\
\text { Retrieval }\end{array}$ & INEEL & 1 & Safety \\
\hline SR00-2027 & $\begin{array}{l}\text { Demonstrate Alternative Filtration Technologies } \\
\text { to Replace HEPA Filters }\end{array}$ & SRS & 3 & Safety \\
\hline 1.1.3 & Characterize Waste & & & \\
\hline RL-WT01 & $\begin{array}{l}\text { Technetium-99 Analysis in Hanford Tank Waste } \\
\text { and Contaminated Tank Farm Areas }\end{array}$ & Hanford & 2 & Characterization \\
\hline RL-WT09 & $\begin{array}{l}\text { Representative Sampling and Associated Analysis } \\
\text { to Support Operations and Disposal }\end{array}$ & Hanford & 2 & Characterization \\
\hline RL-WT013 & $\begin{array}{l}\text { Establish Retrieval Performance Evaluation } \\
\text { Criteria }\end{array}$ & Hanford & 2 & Characterization \\
\hline RL-WT065 & $\begin{array}{l}\text { Direct Inorganic and Organic Analyses of } \\
\text { High-Level Waste }\end{array}$ & Hanford & 2 & Characterization \\
\hline RL-WT083 & Rapid PCB Screening Technology & Hanford & 1 & Characterization \\
\hline RL-WT031-S & Rapid Waste Characterization & Hanford & 2 & Characterization \\
\hline RL-WT032-S & $\begin{array}{l}\text { Monitoring of Key Waste Physical Properties } \\
\text { During Retrieval and Transport }\end{array}$ & Hanford & 3 & Characterization \\
\hline RL-WT052-S & $\begin{array}{l}\text { Characterization of Organic Species in Waste Feed } \\
\text { to LAW and HLW Treatment Facilities }\end{array}$ & Hanford & 1 & Characterization \\
\hline ID-2.1.16 & $\begin{array}{l}\text { Decontamination Facility/Analytical Facility } \\
\text { Waste Reduction }\end{array}$ & INEEL & 1 & Characterization \\
\hline $\mathrm{ID}-2.1 .26$ & $\begin{array}{l}\text { Direct Tank Sampler for Tank Solution } \\
\text { Characterization }\end{array}$ & INEEL & 1 & Characterization \\
\hline ID-2.1.43 & $\begin{array}{l}\text { Certify LDUA Sampler as EPA-Approved Method } \\
\text { of Sampling Tank Heel Liquids }\end{array}$ & INEEL & 1 & Characterization \\
\hline ID-2.1.44 & $\begin{array}{l}\text { Certify LDUA Sampler as EPA-Approved Method } \\
\text { of Sampling Tank Heel Solids }\end{array}$ & INEEL & 1 & Characterization \\
\hline ID-2.1.67 & High Level Waste Slurry Handling & INEEL & 1 & Characterization \\
\hline ID-2.1.72 & Alternate Heel Sampling Systems & INEEL & 1 & Characterization \\
\hline ORTK-04 & Sludge Mixing and Slurry Transport & ORR & 1 & Characterization \\
\hline SR00-2037 & Tank Heel Removal/Closure Technology & SRS & 1 & Characterization \\
\hline SR00-2044 & In-Situ Technology for Waste Characterization & SRS & 3 & Characterization \\
\hline
\end{tabular}


Table A.2. Continued

\begin{tabular}{|c|c|c|c|c|}
\hline PE\# & Problem Element Title & Site & $\begin{array}{l}\text { Need } \\
\text { Pri }\end{array}$ & Function \\
\hline SR00-2054-S & $\begin{array}{l}\text { Develop Improved Radiochemical Analysis for } \\
\text { High Ionic Strength }\end{array}$ & SRS & 3 & Characterization \\
\hline OH-WV-906 & $\begin{array}{l}\text { Radioactivity Measurement of High-Level Waste } \\
\text { Tank Residuals }\end{array}$ & WVDP & 2 & Characterization \\
\hline 1.1.3.1 & Characterize Waste In Situ & & & \\
\hline 1.1.3.2 & Sample Waste & & & \\
\hline 1.1.3.3 & Analyze Waste & & & \\
\hline $\begin{array}{l}\mathrm{ID}-2.1 .31 \\
\mathbf{1 . 1 . 4}\end{array}$ & $\begin{array}{l}\text { Characterization of Entrainable Solids in Tank Wa } \\
\text { Reduce Waste Volume }\end{array}$ & steINEEL & 1 & Characterization \\
\hline ID-2.1.25 & Ion-Exchange System for Water Runoff & INEEL & 1 & Pretreatment \\
\hline ID-2.1.29 & $\begin{array}{l}\text { Evaluate Chloride Corrosion Potential } \\
\text { (LET\&D/PEWE/Future Processes) }\end{array}$ & INEEL & 1 & ESP \\
\hline ID-2.1.30 & $\begin{array}{l}\text { Remove/Treat Chlorides } \\
\text { (LET\&D/PEWE/Future Processes) }\end{array}$ & INEEL & 1 & ESP \\
\hline ID-2.1.36 & Mercury Removal from Liquid Wastes & INEEL & 1 & ESP \\
\hline ID-2.1.41 & HLW Process Offgas Treatment & INEEL & 1 & Immobilization \\
\hline ID-2.1.56 & Mercury Treatment for Aluminum Calcine & INEEL & 1 & ESP \\
\hline ORTK-05 & Tank Sludge and Supernatant Separations & ORR & 1 & Pretreatment \\
\hline ORTK-11 & Tank Supernatant Pretreatment & ORR & 1 & Pretreatment \\
\hline SR00-1011 & $\begin{array}{l}\text { Demonstrate Evaporation Technologies to Reduce } \\
\text { Generation of Secondary Waste Volume from Con }\end{array}$ & $\begin{array}{c}\text { SRS } \\
\text { solidated I }\end{array}$ & $\stackrel{2}{2}$ & $\begin{array}{l}\text { Pretreatment } \\
\text { acility }\end{array}$ \\
\hline SR00-2033 & $\begin{array}{l}\text { Provide Alternative Processing and/or } \\
\text { Concentration Methods for DWPF Recycle Aqueo }\end{array}$ & $\begin{array}{l}\text { SRS } \\
\text { us Streams }\end{array}$ & 2 & Pretreatment \\
\hline 1.1.4 & Reduce Waste Volume & & & \\
\hline 1.1.4.1 & Reduce Source Streams & & & \\
\hline 1.1.4.2 & Reduce Recycle Streams & & & \\
\hline 1.2 & Process Waste & & & \\
\hline 1.2 .1 & Retrieve Waste & & & \\
\hline 1.2.1.1 & Deploy Equipment & & & \\
\hline 1.2.1.2 & Mobilize Bulk and Heel Wastes & & & \\
\hline RL-WT013 & Establish Retrieval Performance Evaluation Criteri & aHanford & 2 & Retrieval \\
\hline RL-WT060 & Better Waste Mixing Mobilization & Hanford & 2 & Retrieval \\
\hline RL-WT063 & $\begin{array}{l}\text { PHMC Retrieval and Closure - Hanford SST } \\
\text { Saltcake Dissolution Retrieval }\end{array}$ & Hanford & 2 & Retrieval. \\
\hline RL-WT064 & $\begin{array}{l}\text { PHMC Retrieval and Closure - Hanford } \\
\text { Past Practice Sluicing }\end{array}$ & Hanford & 1 & Retrieval \\
\hline RL-WT054-S & Solids Yield During Mixer Pump Mobilization & Hanford & 2 & Retrieval \\
\hline RL-WT077-S & Improvements to Salt Well Pumping & Hanford & 2 & Retrieval \\
\hline ID-2.1.50 & Solids Waste (Calcine) Retrieval & INEEL & 1 & Retrieval \\
\hline $\mathrm{D}-2.1 .67$ & High Level Waste Slurry Handling & INEEL & 1 & Retrieval \\
\hline ID-2.1.69 & Solids Waste (Calcine) Retrieval from CSSF1 & INEEL & 1 & Retrieval \\
\hline ORTK-02 & Tank Solid Waste Retieval & ORR & 1 & Retrieval \\
\hline SR00-2028 & Alternative Waste Removal Technology & SRS & 1 & Retrieval \\
\hline SR00-2037 & Tank Heel Removal/Closure Technology & SRS & 1 & Retrieval \\
\hline SR00-2041 & Develop Advanced Mixing Technology & SRS & 3 & Retrieval \\
\hline OH-WV-905 & Retrieval of Tank Heels & WVDP & 1 & Retrieval \\
\hline 1.2.1.4 & Transfer Waste & & & \\
\hline RL-WT023 & $\begin{array}{l}\text { Prediction of Solid Phase Formation in Static } \\
\text { and Dynamic Hanford Tank Waste Solutions }\end{array}$ & Hanford & 2 & Retrieval \\
\hline RL-WT062 & Variable Suction Level Transfer Pump & Hanford & 1 & Retrieval \\
\hline RL-WT040-S & Mechanisms of Line Plugging & Hanford & 2 & Retrieval \\
\hline ID-2.1.67 & High Level Waste Slurry Handling & INEEL & 1 & Retrieval \\
\hline SR00-2037 & Tank Heel Removal/Closure Technology & SRS & 1 & Retrieval \\
\hline SR00-2039 & Methods to Unplug Waste Transfer Lines & SRS & 2 & Retrieval \\
\hline
\end{tabular}


Table A.2. Continued

\begin{tabular}{|c|c|c|c|c|}
\hline PE\# & Problem Element Title & Site & $\begin{array}{l}\text { Need } \\
\text { Pri }\end{array}$ & Function \\
\hline 1.2.1.5 & Detect and Mitigate Leaks & & & \\
\hline RL-WT026 & $\begin{array}{l}\text { Tank Leak Detection Systems for Underground } \\
\text { Single-Shell Waste Storage Tanks (SSTs) }\end{array}$ & Hanford & 1 & Safety \\
\hline RL-WT027 & Tank Leak Mitigation Systems & Hanford & 1 & Safety \\
\hline SR00-2037 & Tank Heel Removal/Closure Technology & SRS & 1 & Safety \\
\hline 1.2.1.6 & Transfer Waste & & & \\
\hline 1.2.1.7 & \multicolumn{4}{|c|}{ Integrate Retrieval and Pretreatment Technology Systems } \\
\hline 1.2.1.8 & \multicolumn{4}{|c|}{ Mobilize Heel } \\
\hline 1.2.2 & Pretreat Waste & \multicolumn{2}{|l|}{$\therefore$} & \\
\hline 1.2.2.1 & Calcine Waste & & & \\
\hline 1.2.2.2 & Dissolve Waste & & & \\
\hline ID-2.1.51 & $\begin{array}{l}\text { Develop Calcine Dissolution Kinetics for } \\
\text { Solid/Liquid Equilibria }\end{array}$ & INEEL & 1 & Pretreatment \\
\hline ID-2.1.52 & \multicolumn{2}{|c|}{ Characterization of Solids from Calcine Dissolution INEEL } & 1 & Pretreatment \\
\hline 1.2.2.3 & \multicolumn{3}{|c|}{ Prepare Retrieved Waste for Transfer and Pretreatment } & \\
\hline RL-WT023 & $\begin{array}{l}\text { Prediction of Solid Phase Formation in Static } \\
\text { and Dynamic Hanford Tank Waste Solutions }\end{array}$ & Hanford & 2 & Pretreatment \\
\hline RL-WT063 & $\begin{array}{l}\text { PHMC Retrieval and Closure - Hanford SS } \\
\text { Saltcake Dissolution Retrieval }\end{array}$ & Hanford & 2 & Pretreatment \\
\hline RL-WT071 & \multicolumn{2}{|c|}{ ESP Modeling Support for the Back Dilution of Tank 241-SY-101 } & $101^{1}$ & Pretreatment \\
\hline RL-WT040-S & Mechanisms of Line Plugging & Hanford & 2 & Pretreatment \\
\hline RL-WT049-S & $\begin{array}{l}\text { Effect of Processing on Gas Release, Waste } \\
\text { Sedimentation, Rheological, and Other Behaviors }\end{array}$ & Hanford & 3 & Pretreatment \\
\hline RL-WT075-S & HLW Solid Phase Characterization & Hanford & 1 & Pretreatment \\
\hline RL-WT078-S & Plutonium Segregation and Association in HLW & Hanford & 2 & Pretreatment \\
\hline ID-2.1.15 & Neutralization of Newly Generated Liquid Wastes & INEEL & 1 & Pretreatment \\
\hline ORTK-04 & Sludge Mixing and Slurry Transport & ORR & 1 & Pretreatment \\
\hline SR00-2037 & Tank Heel Removal/Closure Technology & SRS & 1 & Pretreatment \\
\hline SR00-2039 & Methods to Unplug Waste Transfer Lines & SRS & 2 & Pretreatment \\
\hline 1.2.2.4 & Clarify Liquid Stream & & & \\
\hline $\mathrm{ID}-2.1 .64$ & $\begin{array}{l}\text { Solid-Liquid Separation Equipment Development } \\
\text { and Application }\end{array}$ & INEEL & 1 & Pretreatment \\
\hline 1.2.2.5 & Remove Radionuclides & & & \\
\hline RL-WT082 & Crystalline Silicotitanate Non-Elutable Sorbent & Hanford & 2 & Pretreatment \\
\hline ID-2.1.06 & $\begin{array}{l}\text { TRU, Cs and Sr Removal from High Activity } \\
\text { Wastes }\end{array}$ & INEEL & 1 & Pretreatment \\
\hline $\mathrm{ID}-2.1 .28$ & $\begin{array}{l}\text { Cs and Sr Removal from Newly Generated } \\
\text { Liquid Waste }\end{array}$ & INEEL & 1 & Pretreatment \\
\hline ID-2.1.56 & Mercury Treatment for Aluminum Calcine & INEEL & 1 & Pretreatment \\
\hline ID-2.1.68 & $\begin{array}{l}\text { Technetium Removal from INEEL High Level } \\
\text { Waste }\end{array}$ & INEEL & 1 & Pretreatment \\
\hline ORTK-11 & Tank Supernatant Pretreatment & ORR & 1 & Pretreatment \\
\hline SR00-2034 & Second Generation Salt Feed Preparation & SRS & 1 & Pretreatment \\
\hline SR00-2053-S & $\begin{array}{l}\text { Develop an Alternative Sorbent to Replace } \\
\text { Monosodium Titanate for Sr and Actinide Removal }\end{array}$ & SRS & 3 & Pretreatment \\
\hline 1.2.2.6 & Integrate Pretreatment and LLW Immobilization & in Technol & & \\
\hline ID-2.1.24 & $\begin{array}{l}\text { Integration/Optimization of High Activity Waste/ } \\
\text { Low Activity Waste Process Flowsheet }\end{array}$ & INEEL & 1 & Immobilization \\
\hline ID-2.1.65 & Treatment/Disposition of Removed Tank Solids & INEEL & 1 & Immobilization \\
\hline & $\begin{array}{l}\text { Increase in Applicability/Efficiency of High-Level } \\
\text { Waste Planning Tool }\end{array}$ & SRS & 3 & Immobilization \\
\hline $\begin{array}{l}\text { 1.2.2.7 } \\
\text { RL-WT024 }\end{array}$ & $\begin{array}{l}\text { Process Sludge } \\
\text { Enhanced Sludge Washing Process Data }\end{array}$ & Hanford & 2 & Pretreatment \\
\hline
\end{tabular}


Table A.2. Continued

\begin{tabular}{|c|c|c|c|c|}
\hline PE\# & Problem Element Title & Site & $\begin{array}{l}\text { Need } \\
\text { Pri }\end{array}$ & Function \\
\hline$\overline{R L}-W T 070$ & $\begin{array}{l}\text { Uncertainty Estimation of Hanford Best Basis } \\
\text { Toxic Waste Inventory, Concentration, Phase and W }\end{array}$ & $\begin{array}{l}\text { Hanford } \\
\text { Waste Type }\end{array}$ & $\overline{1}$ & $\overline{\text { Pretreatment }}$ \\
\hline RL-WT038-S & Process Models for Sludge Treatment & Hanford & 2 & Pretreatment \\
\hline RL-WT037-S & Sludge Treatment & Hanford & 2 & Pretreatment \\
\hline RL-WT078-S & Plutonium Segregation and Association in HLW & Hanford & 2 & Pretreatment \\
\hline ORTK-05 & Tank Sludge and Supernatant Separations & ORR & 1 & Pretreatment \\
\hline SR00-2052 & $\begin{array}{l}\text { Aluminum Dissolution from HAW Sludge and } \\
\text { Its Impact on Downstream Salt Processing }\end{array}$ & SRS & 2 & Pretreatment \\
\hline 1.2.2.8 & Prepare Pretreated Waste for Immobilization & & & \\
\hline 1.2.2.9 & Monitor \& Control Pretreatment Process & & & \\
\hline 1.2 .3 & Immobilize Waste & & & \\
\hline 1.2.3.1 & Process LLW & & & \\
\hline ID-2.1.23 & Low-Activity Wasteform Qualification & INEEL & 1 & Immobilization \\
\hline ID-2.1.28 & $\begin{array}{l}\text { Cs and Sr Removal from Newly Generated } \\
\text { Liquid Waste }\end{array}$ & INEEL & 1 & Immobilization \\
\hline ID-2.1.35 & $\begin{array}{l}\text { Direct Immobilization of INTEC Sodium-Bearing } \\
\text { and Newly Generated Liquid Wastes }\end{array}$ & INEEL & 1 & Immobilization \\
\hline ID-2.1.38 & Conditioning of Low Activity Waste for Treatment & INEEL & 1 & Immobilization \\
\hline ID-2.1.40 & $\begin{array}{l}\text { Low Activity Waste Grout Sorbent Addition to } \\
\text { Reduce Leachability }\end{array}$ & INEEL & 1 & Immobilization \\
\hline ID-2.1.66 & $\begin{array}{l}\text { Treatment/Disposition of Spent Ion Exchange } \\
\text { Resins }\end{array}$ & INEEL & 1 & Immobilization \\
\hline ORTK-06 & Tank Sludge Supernatant Immobilization & ORR & 1 & Immobilization \\
\hline 1.2.3.1.1 & Monitor \& Control LLW Immobilization Process & & & \\
\hline 1.2.3.1.2 & Prepare LLW Feed & & & \\
\hline 1.2.3.1.3 & Immobilize LLW Feed & & & \\
\hline 1.2.3.1.4 & Treat LLW Offgas & & & \\
\hline 1.2.3.1.5 & Dispose of LLW & & & \\
\hline 1.2.3.2 & Process HLW & & & \\
\hline RL-WT080 & Advanced/Improved Vitrification & Hanford & 2 & Immobilization \\
\hline RL-WT081 & Sulfate Accumulation in Low Activity Waste & Hanford & 2 & Immobilization \\
\hline RL-WT085 & $\begin{array}{l}\text { Extension of Glass Properties Model to Lower } \\
\text { Silica Compositions }\end{array}$ & Hanford & 1 & Immobilization \\
\hline ID-2.1.19 & $\begin{array}{l}\text { EPA Methods Sample Collection and Analysis } \\
\text { Verification/Development }\end{array}$ & INEEL & 1 & Characterization \\
\hline ID-2.1.49 & $\begin{array}{l}\text { Acceptance Criteria for High Activity Waste/ } \\
\text { Low Activity Waste }\end{array}$ & INEEL & 1 & Immobilization \\
\hline ID-2.1.57 & Conditioning of HAW for Treatment & INEEL & 1 & Immobilization \\
\hline ID-2.1.58 & HAW Immobilization & INEEL & 1 & Immobilization \\
\hline ID-2.1.66 & $\begin{array}{l}\text { Treatment/Disposition of Spent Ion Exchange } \\
\text { Resins }\end{array}$ & INEEL & 1 & Immobilization \\
\hline SR00-2032 & $\begin{array}{l}\text { Optimize Melter Glass Chemistry and Increase } \\
\text { Waste Loading }\end{array}$ & SRS & 2 & Immobilization \\
\hline SR00-2036 & Develop Improved HLW Melter & SRS & 3 & Immobilization \\
\hline 1.2.3.2.1 & Monitor \& Control HLW Immobilization Process & & & \\
\hline 1.2.3.2.2 & Prepare Secondary Waste from Pre & & & \\
\hline 1.2.3.2.3 & Prepare $\mathbf{S}$ & & & \\
\hline 1.2.3.2.4 & Immobilize HLW Stream & & & \\
\hline 1.2 .3 .2 .5 & $\begin{array}{l}\text { Treat HLW Offgas } \\
\text { Store Waste Forms and Close Tanks }\end{array}$ & & & \\
\hline 1.3.1 & Close Tanks & & & \\
\hline RL-WT013 & $\begin{array}{l}\text { Establish Retrieval Performance Evaluation } \\
\text { Criteria }\end{array}$ & Hanford & 2 & Closure \\
\hline RL-WT061 & Reactive Barriers to Contaminant Migration & Hanford & 2 & Closure \\
\hline RL-WT068 & Radionuclide Source Term from Tank Residuals & Hanford & 2 & ESP \\
\hline
\end{tabular}


Table A.2. Continued

\begin{tabular}{|c|c|c|c|c|}
\hline & & & Need & \\
\hline PE\# & Problem Element Title & $\underline{\text { Site }}$ & $\underline{\text { Pri }}$ & Function \\
\hline RL-WT069 & $\begin{array}{l}\text { Value of Information Decision Analysis for } \\
\text { Tank Farm Closure }\end{array}$ & Hanford & 2 & Closure \\
\hline RL-WT046-S & Getter Materials & Hanford & 2 & Closure \\
\hline ID-2.1.39 & $\begin{array}{l}\text { Acceptance Criteria for LAW Disposal in } \\
\text { Underground Storage Tanks }\end{array}$ & INEEL & 1 & Closure \\
\hline $\mathrm{ID}-2.1 .42$ & Acceptance Criteria for Tank Closure & INEEL & 1 & Closure \\
\hline ID-2.1.45 & Acceptance Criteria for Grouting Tank Heels & INEEL & 1 & Closure \\
\hline $\mathrm{ID}-2.1 .46$ & Management of Tank Heel Liquids & INEEL & 1 & Closure \\
\hline $\mathrm{ID}-2.1 .47$ & Management of Tank Heel Solids & INEEL & 1 & Closure \\
\hline ID-2.1.48 & $\begin{array}{l}\text { Wasteform Qualification for Low-Activity Waste } \\
\text { in Underground Storage Tanks }\end{array}$ & INEEL & 1 & Closure \\
\hline $\mathrm{ID}-2.1 .62$ & Acceptance Criteria for Bin Set Closure & INEEL & 1 & Closure \\
\hline ORTK-09 & Tank Closure & ORR & 3 & Closure \\
\hline SR00-2051 & $\begin{array}{l}\text { Technology to Mitigate Effects of Technetium } \\
\text { Under Tank Closure Conditions }\end{array}$ & SRS & 2 & ESP \\
\hline SR00-3022 & $\begin{array}{l}\text { In-Situ Grouting and/or Retrieval of Waste from } \\
\text { Underground Tanks (Formerly Used for the Storag }\end{array}$ & $\begin{array}{l}\text { SRS } \\
\text { ef Radio }\end{array}$ & $\begin{array}{l}2 \\
\text { tive Sol }\end{array}$ & $\begin{array}{l}\text { Closure } \\
\text { nts) }\end{array}$ \\
\hline SR00-2049-S & $\begin{array}{l}\text { Technetium Chemistry Under Waste Removal } \\
\text { Conditions }\end{array}$ & SRS & 1 & ESP \\
\hline $\begin{array}{l}\text { OH-WV-914 } \\
\text { 1.3.1.1 }\end{array}$ & $\begin{array}{l}\text { Development of Grout for In-Situ Closure } \\
\text { Monitor Tank }\end{array}$ & WVDP & 1 & Closure \\
\hline 1.3.1.2 & Characterize Heels & & & \\
\hline 1.3.1.3 & Define Closure Criteria & & & \\
\hline 1.3.1.4 & Treat Supernate in Place & & & \\
\hline 1.3.1.5 & Treat Heel in Place & & & \\
\hline 1.3.1.6 & Detect Leaks & & & \\
\hline 1.3.1.7 & Stabilize Tank for Closure & & & \\
\hline 1.3.1.8 & Monitor Site & & & \\
\hline 1.3.2 & Dispose of LLW & & & \\
\hline RL-WT015 & $\begin{array}{l}\text { Standard Method for Determining Waste Form } \\
\text { Release Rate }\end{array}$ & Hanford & 1 & Immobilization \\
\hline RL-WT016 & Glass Monolith Surface Area & Hanford & 1 & Immobilization \\
\hline RL-WT017 & Long-Term Testing of Surface Barrier & Hanford & 2 & Closure \\
\hline RL-WT018 & Testing of Sand-Gravel Capillary Barrier & Hanford & 2 & Closure \\
\hline RL-WT029 & Data and Tools for Performance Assessments & Hanford & 2 & Closure \\
\hline RL-WT066 & $\begin{array}{l}\text { Compositional Dependence of the Long Term } \\
\text { Performance of Glass as a Low-Activity Waste For }\end{array}$ & $\begin{array}{l}\text { Hanford } \\
\mathrm{rm}\end{array}$ & 2 & Immobilization \\
\hline RL-WT035-S & $\begin{array}{l}\text { Moisture Flow and Contaminant Transport in } \\
\text { Arid Conditions }\end{array}$ & Hanford & 1 & Closure \\
\hline RL-WT043-S & $\begin{array}{l}\text { Effect of Human and Natural Influences on } \\
\text { Long-Term Water Distribution }\end{array}$ & Hanford & 1 & Closure \\
\hline RL-WT044-S & Distribution of Recharge Rates & Hanford & 1 & Closure \\
\hline RL-WT045-S & $\begin{array}{l}\text { Vadose Zone Flow Simulation Tool Under } \\
\text { Arid Conditions }\end{array}$ & Hanford & 1 & Closure \\
\hline RL-WT056-S & Half-Lives of Se-79 and Sn-126 & Hanford & 1 & Closure \\
\hline ID-2.1.70 & Low-Activity Waste Biodegradation Test & INEEL & 1 & Closure \\
\hline OH-WV-904 & High Level Waste Tank Closure & WVDP & 1 & Closure \\
\hline 1.3.2.1 & Monitor Low Level Waste for Acceptance & & & \\
\hline 1.3.2.2 & Determine Performance of Waste Form & & & \\
\hline 1.3.2.3 & Provide Disposal System & & & \\
\hline 1.3.3 & Store and Dispose HLW & & & \\
\hline 1.3.3.1 & Provide Interim Storage HLW & & & \\
\hline 1.3.3.2 & Provide Shipping Facilities & & & \\
\hline 1.3.3.3 & Monitor High Level Waste for Acceptance & & & \\
\hline
\end{tabular}


Table A.2. Continued

\begin{tabular}{|c|c|c|c|c|}
\hline PE\# & Problem Element Title & $\underline{\text { Site }}$ & $\begin{array}{l}\text { Need } \\
\text { Pri }\end{array}$ & Function \\
\hline 1.4 & Decontamination and Decommissioning & & & \\
\hline RL-WT021 & $\begin{array}{l}\text { Cleaning, Decontaminating and Upgrading } \\
\text { Hanford Pits }\end{array}$ & Hanford & 2 & Retrieval \\
\hline ID-2.1.16 & $\begin{array}{l}\text { Decontamination Facility/Analytical Facility } \\
\text { Waste Reduction }\end{array}$ & INEEL & 1 & Pretreatment \\
\hline ID-2.1.17 & Develop New Filter Leach Process & INEEL & 1 & Pretreatment \\
\hline SR00-2029 & Alternate DWPF Canister Decon Technology & SRS & 3 & Immobilization \\
\hline SR00-2031 & $\begin{array}{l}\text { Develop Remote Technology to Improve } \\
\text { DWPF Operations }\end{array}$ & SRS & 3 & Retrieval \\
\hline SR00-2037 & Tank Heel Removal/Closure Technology & SRS & 1 & Char/Retrieval \\
\hline SR00-2040 & $\begin{array}{l}\text { Demonstrate Remote Decommissioning and } \\
\text { Disassembly of High Level Waste Processing E }\end{array}$ & $\begin{array}{l}\text { SRS } \\
\text { pment }\end{array}$ & 3 & Immobilization \\
\hline OH-WV-902 & $\begin{array}{l}\text { Decontamination of High-Level Waste (HLW) } \\
\text { Canisters (WVDP-2-99) }\end{array}$ & WVDP & 1 & Immobilization \\
\hline OH-WV-903 & $\begin{array}{l}\text { Vitrification Expended Material Processing } \\
\text { (WVDP-3-99) }\end{array}$ & WVDP & 1 & Immobilization \\
\hline OH-WV-904 & High Level Waste Tank Closure & WVDP & 1 & Robotics \\
\hline OH-WV-908 & $\begin{array}{l}\text { Decontamination of High-Level Waste } \\
\text { Contaminated Equipment }\end{array}$ & WVDP & 3 & Robotics \\
\hline
\end{tabular}


Table A.3. Tanks Focus Area FY 2001 - FY 2002 Integrated Priority Listing

\begin{tabular}{|c|c|c|c|c|c|c|}
\hline \multirow{2}{*}{$\begin{array}{l}\text { TFA } \\
\text { Pri }\end{array}$} & \multirow{2}{*}{$\begin{array}{l}\text { TFA } \\
\text { Resp }\end{array}$} & \multirow[b]{2}{*}{ Technical Response Title } & \multicolumn{3}{|c|}{ Benefiting Sites } & \multirow[b]{2}{*}{ Site Needs Included } \\
\hline & & & \begin{tabular}{l|l|} 
Han & INEEL \\
\end{tabular} & \begin{tabular}{|l|l|} 
ORR & SRS \\
\end{tabular} & WVDP & \\
\hline 1 & A9143 & HLW Tank Corrosion Control and Monitoring & & & & ORTK-01, RL-WT04, RL-WT079-S, SR00-2045 \\
\hline 2 & A9586 & CIF Evaporator & & & & ORTK-05, ORTK-11, SR00-1011 \\
\hline 3 & A9768 & Specify and Enhance Design of HLW Glass Melters & & & & $\begin{array}{l}\text { ID-2.1.57, ID-2.1.58, ID-2.1.66, RL-WT080, SR00- } \\
2036\end{array}$ \\
\hline 4 & A9554 & Hanford Tank Waste Chemistry & & & & $\begin{array}{l}\text { ORTK-04, RL-WT023, RL-WT040-S, } \\
\text { RL-WT049-S, RL-WT063, RL-WT071, } \\
\text { RL-WT075-S, RL-WT078-S, SR00-2037, } \\
\text { SR00-2039 }\end{array}$ \\
\hline 5 & $\mathrm{A9773}$ & Improve Waste Loading in HLW Glass & & & & $\begin{array}{l}\text { ID-2.1.58, ID-2.1.66, RL-WT80, RL-WT08I, } \\
\text { RL-WT084, SR00-2032, SR00-2036 }\end{array}$ \\
\hline 6 & A9570 & Salt Disposition & & & & ID-2.1.28, ORTK-11, RL-WT082, SR00-2034 \\
\hline 7 & A9709 & Waste Treatment Process Flowsheet Model & & & & ID-2.1.24, ID-2.1.65, SR00-2055 \\
\hline 8 & A9365 & Waste Transfer Pumping & & & & ID-2.1.67, RL-WT062, SR00-2037 \\
\hline 9 & $\overline{\mathrm{A9555}}$ & Sludge Washing and Dissolution & & & & $\begin{array}{l}\text { ORTK-05, RL-WT024, RL-WT037-S, } \\
\text { RL-WT038-S, RL-WT070, RL-WT078-S, } \\
\text { SR00-2052 }\end{array}$ \\
\hline 10 & A9923 & Enhanced Grout Formulations for Tank Closure & & & & $\begin{array}{l}\text { ID-2.1.46, ID-2.1.47, OH-WV-914, ORTK-09, } \\
\text { SR00-3022 }\end{array}$ \\
\hline 11 & A9175 & Tank Integrity Inspection Techniques & & & & $\begin{array}{l}\text { ID-2.1.20, OH-WV-907, ORTK-01, RL-WT05, } \\
\text { RL-WT022, RL-WT067, SR00-2035, SR00-2037, } \\
\text { SR00-2050-S }\end{array}$ \\
\hline 12 & A9376 & Waste Transfer Line Plugging Prevention and Unplugging Methods & & & & RL-WT023, RL-WT040-S, SR00-2039 \\
\hline 13 & A9777 & Remote Disassembly of HLW Melters and Other Processing Equipment & & & & OH-WV-903, SR00-2040 \\
\hline 14 & A9719 & Conditioning and Immobilization of Low-Activity Waste & & & & $\begin{array}{l}\text { ID-2.1.23, ID-2.1.28, ID-2.1.35, ID-2.1.38, } \\
\text { ID-2.1.40, ID-2.1.66, ORTK-06 }\end{array}$ \\
\hline 15 & A9171 & Altemative Air Filtration Technology & & & & ID-2.1.27, SR00-2027 \\
\hline 16 & A9361 & Heel Retrieval from Obstructed Tanks & & & & ID-2.1.67, OH-WV-905 \\
\hline 17 & A9363 & Chemical Cleaning of Tanks & & & & SR00-2037 \\
\hline $1 \overline{8}$ & Aि203 & Residual Waste Sampling & (2) & & & ID-2.1.72, RL-WT013, SR00-2037 \\
\hline 19 & A9362 & Salt Cake Dissolution Retrieval & & (1) & & RL-WT063, RL-WT077-S, SR00-2028 \\
\hline 20 & A9157 & Tank Leak Mitigation & & & & RL-WT027, SR00-2037 \\
\hline 21 & A9246 & Waste Sampling and At-Tank Analysis & & & & ID-2.1.26, ID-2.1.43, ID-2.1.44, RL-WT09 \\
\hline 22 & A9584 & Cross-Flow Filtration & & & & ID-2.1.64 \\
\hline 23 & A99352 & Remote Systems for Pit Operations and Maintenance & & & & RL-WT021, SR00-2037 \\
\hline 24 & A9501 & INEEL Integrated Radionuclide Separations Process & & & & ID-2.1.06, ID-2.1.56, ID-2.1.68 \\
\hline
\end{tabular}

\begin{tabular}{|l|l|}
\hline & $=$ Primary Benefit \\
\hline $3_{3 x}$ & $=$ Secondary Benefit \\
& $=$ No benefit or benefit undetermined \\
\hline
\end{tabular}




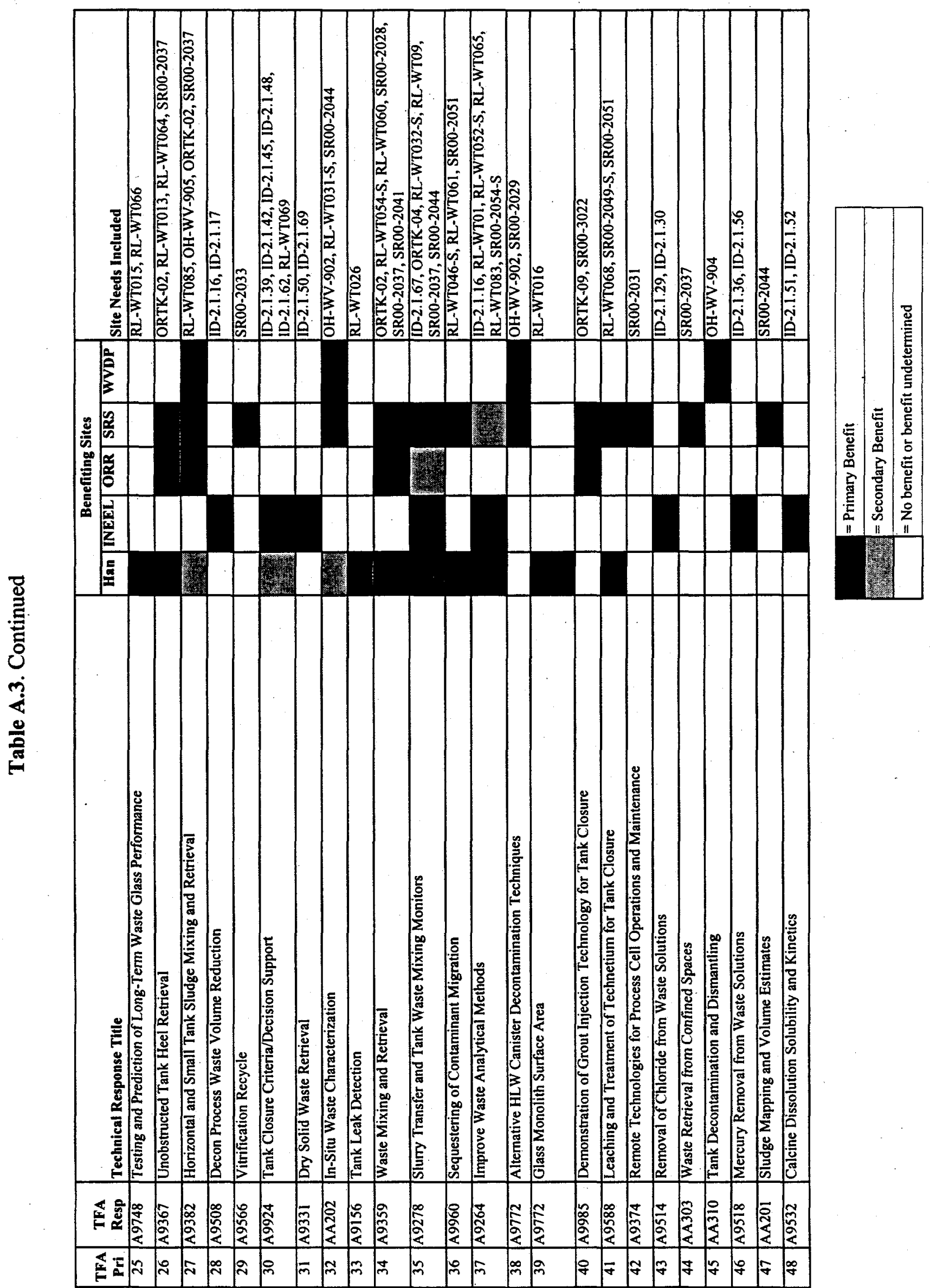




\section{Site: Hanford}

Site Need ID: RL-WT01

Site Need Title: Technetium-99 Analysis in Hanford Tank Waste and Contaminated Tank Farm Areas

Need Summary: An accurate, robust production laboratory method for the measurement of technetium-99 ( $99 \mathrm{Tc}$ ) concentration in Hanford Site waste tank matrices and in soils from the vadose zone surrounding the tanks is needed. The method must provide a high level of confidence in the $99 \mathrm{Tc}$ concentrations because data are important in risk-based assessments. To obtain this level of confidence, verification of method performance needs to be done by the use of independent methods and/or by interlaboratory comparisons on actual waste samples between U.S. Department of Energy (DOE) Sites.

Technical Disposition: The TFA responded to this need within the following technical response(s):

- A9264, Improve Waste Analytical Methods, TFA priority \#37.

Site Need ID: RL-WT04

Site Need Title: Double-Shell Tank Corrosion Monitoring

Need Summary: Corrosion monitoring of double-shell tanks (DSTs) is currently provided by process knowledge and tank sampling. Tanks found to be within chemistry specification limits are considered to be not at risk for excessive corrosion damage. There have been no direct corrosion monitoring systems for DSTs in use at the Hanford Site. As many as 4 low hydroxide (out of corrosion specification) tanks continue to be operated. In fiscal year 1999, DST 241-AN-105 was discovered to have wall thinning that cannot be explained by existing corrosion chemistry models. This indicates that this system is inadequate to support corrosion control. Tank samples are infrequent and their analysis difficult and expensive. Process knowledge is complicated by waste streams that are exempt from the corrosion control specifications. In-tank, real-time measurement of the corrosive characteristics of the tank wastes is needed to improve control of corrosion processes. This need supports RPP Program Logic "Conduct Tank Farms Safe Operations" and "Conduct Reduced Mortgage Tank Farm Safe Operations." Corrosion monitoring is discussed in the Safe Storage Technical Basis Review, Activity Number 190.N45.

Technical Disposition: The TFA responded to this need within the following technical response(s):

- A9143, HLW Tank Corrosion Control and Monitoring, TFA priority \#1.

Site Need ID: RL-WT05

Site Need Title: Remote Inspection of High-Level Waste Single-Shell Tanks Need Summary: The Hanford Federal Facility Agreement and Consent Order (Tri Party Agreement) schedule requires retrieval of waste in the single-shell tanks (SST). To meet this schedule, a retrieval method needs to be selected to retrieve the waste for processing. A nondestructive evaluation (NDE) of the tank needs to be performed prior to the selection of a retrieval method to assure successful retrieval of the waste from the tank.

Technical Disposition: The TFA responded to this need within the following technical response(s): 
- A9175, Tank Integrity Inspection Techniques, TFA priority \#11.

- AA1S1, Pre-Closure Interim Tank Maintenance, TFA strategic task.

Site Need ID: RL-WT09

Site Need Title: Representative Sampling and Associated Analysis to Support Operations and Disposal

Need Summary: The Hanford need is for a RCRA-compatible sampling system and associated rapid analysis of feeds which are to be staged for transfer as feed for the ORP Privatization Contractor. Feed for Privatization Phase I immobilization demonstrations must be sampled prior to transfer to the Privatization Contractor. The samples must be representative of the tank contents. To support the Hanford Privatization Contract, a variable depth or multiple depth sampling system is needed to take representative LAW and HLW samples that will be used to certify the tank contents. The certified tank contents will be needed either for acceptance of the feed by the Privatization Contractor or as a means to determine the additional compensation that the Privatization Contractor will receive. Sampling while the mixer pumps are operating will assure that the samples are representative of the waste batch that is anticipated to be transferred to the Privatization Contractor. An attank analysis system is desired for the Hanford need that measures key waste properties from which the mixing/settling status of a tank waste batch can be assessed.

Other sites have needs that are specific to sampling, sample size, and sampling criteria. For example, INEEL requires a RCRA compatible sampler that can sample their tank wastes while SRS has a requirement for a rapid, fixed depth sampler. The INEEL sampling need is to provide waste samples for assessing the chemical content while the SRS need is to assess the settling status of a tank. (Reference: Alternatives Generation and Analysis for the Phase I Intermediate Waste Feed Staging System Design Requirements, WHC-SD-TWR-AGA-001, Rev. 0).

Technical Disposition: The TFA responded to this need within the following technical response(s):

- A9246, Waste Sampling and At-Tank Analysis, TFA priority \#21.

- A9278, Slurry Transfer and Tank Waste Mixing Monitors, TFA priority \#35.

Site Need ID: RL-WT013

Site Need Title: Establish Retrieval Performance Evaluation Criteria

Need Summary: The Tri-Party Agreement establishes an interim retrieval performance goal to leave no more than $360 \mathrm{ft}^{3}$ of waste in 75 -ft-diameter SSTs, and no more than $30 \mathrm{ft}^{3}$ in 20$\mathrm{ft}$-diameter SSTs. This interim goal is intended to be finalized or modified over time based on demonstrations of retrieval technology, and on evaluation of cost, technical practicability, exposure of workers and public to radiation, and compliance with U.S. Nuclear Regulatory Commission requirements that will establish authority to regulate disposal of the radioactive component of residual waste.

A principal function of waste retrieval is to remove sufficient waste from tanks to permit tank closure. The TWRS Environmental Impact Statement (EIS) evaluated environmental impacts associated with retrieval of waste from SSTs using technologies that are expected to leave residual volumes of waste approximating the interim Tri-Party Agreement retrieval 
performance goal. If residual waste must be retrieved from SSTs as part of closure operations, environmental impacts of such waste retrieval, including impacts on tank waste processing, have not been evaluated.

Technical Disposition: The TFA responded to this need within the following technical response(s):

- A9367, Unobstructed Tank Heel Retrieval, TFA priority \#26.

- A9947, Vadose Zone Characterization Technologies. Previously, the TFA provided Hanford the cone penetrometer technology as a solution for this portion of the need. In discussion with site personnel, the TFA has not identified any additional activities required to satisfy this portion of the need.

- AA203, Residual Waste Sampling, TFA priority \#18.

- AA1S1, Pre-Closure Interim Tank Maintenance, TFA strategic task.

- AA3S2, SST Retrieval from Potential Leaking Tanks, TFA strategic task.

Site Need ID: RL-WT015

Site Need Title: Standard Method for Determining Waste Form Release Rate Need Summary: The release of radionuclides from a waste form and package to the environment results from the interactions between the waste form and water in the disposal system. For the disposal of immobilized low-activity tank waste (ILAW), the waste form and package are expected to be in an extremely dry environment. In such an environment, the release rate is a sensitive function of physical (temperature, water content) and chemical (pH and amount and type of mineral and non-mineral species) environment.

Waste forms are typically developed to minimize the rate of release as measured by a variety of test methods. Current ILAW product specifications require Product Consistency Test (PCT) testing and ANS 16.1 testing of the waste forms, which involve testing the waste form in an environment where water is abundant and where chemical effects are minimized. These test methods will not be representative of the expected disposal system environment at the Hanford Site. A release rate test method yielding results that can be related to the waste form release rate under expected service conditions is needed as a basis for Phase II Privatization ILAW product specifications.

Tests are also used to determine release data for use in the analysis for the assurance that long-term public health and safety will be protected using the proposed disposal method. Such tests must examine a wider set of environmental conditions that product acceptance tests and will form the basis of the Performance Assessment for the disposal action. As shown in the Hanford Immobilized Low-Activity Tank Waste Performance Assessment (DOE/RL-97-69), the contaminant release rate from the waste form is one of the few major factors in the assurance of public health and safety.

Technical Disposition: The TFA responded to this need within the following technical response(s):

- A9748, Testing and Prediction of Long-Term Waste Glass Performance, TFA priority \#25.

- AA7S1, Durability of Multiphase Waste Glasses, TFA strategic task. 
Site Need ID: RL-WT016

Site Need Title: Glass Monolith Surface Area

Need Summary: A method is needed to estimate the surface area of vitrified low-activity waste (LAW). The contaminant release rate from glasses is proportional to the surface area reachable by moving moisture. As glass cools it experiences internal stresses and strains, which may cause the glass to crack and hence increase the surface area on the glass. External stresses (for example, those caused by earthquakes) could also increase surface area. In addition, cracks may expose imperfections in waste form (internal gas pockets, nucleation sites, and devitrification regions) which may cause increased contaminant release rates. Relatively little is known about the long-term behavior of such cracks, yet the total contaminant release must be known (or at least estimated) for thousands of years.

Technical Disposition: The TFA responded to this need within the following technical response(s):

- A9749, Glass Monolith Surface Area, TFA priority \#39.

Site Need ID: RL-WT017

Site Need Title: Long-Term Testing of Surface Barrier

Need Summary: Surface barriers are used over many Hanford environmental restoration and waste management sites and more barriers are expected in the future. Such barriers are used to reduce moisture infiltration and plant and animal intrusion. Short-term testing of barriers has occurred under project-sponsored activities, but long-term studies remain a funding orphan. Project-specific funding at the Hanford Site ended in September 1997. Because the design life of the barrier is 1,000 years, data will be needed on degradation to better understand the validity of the design life estimate. (A similar technology needs statement is also included in the Subsurface Contaminants needs section.)

Technical Disposition: The TFA responded to this need within the following technical response(s):

- A9950, Barriers for Tank/Disposal Facility Closure. Because the Subcon Focus Area infiltration barrier effort is satisfying site needs for a guidance document, the TFA did not develop a separate technical response. The TFA will revisit whether it should develop a response after Hanford has an opportunity to review the Subcon effort and submit revised needs to the TFA.

Site Need ID: RL-WT018

Site Need Title: Testing of Sand-Gravel Capillary Barrier

Need Summary: Water is the driving force behind releasing contaminants from waste forms, then carrying those contaminants to groundwater. Surface moisture barriers (such as the Hanford barrier) have a design life of 1,000 years. Yet, because of the dry conditions at the Hanford Site, moisture infiltration should be minimized for thousands of years.

Unlike a surface barrier, which uses many of the same hydrologic principles, a capillary barrier diverts water away from the protected object underneath rather than storing the water until evaporation or plant transpiration removes the water. Thus the capillary barrier is expected to have a significantly longer life and be more effective than a surface barrier for moisture diversion. Although the principles of sand-gravel capillary barriers are well established, such barriers (especially of ones the size needed for DOE applications) have not 
been extensively tested. Performance data are needed to confirm design parameters and longterm performance estimates.

Technical Disposition: The TFA responded to this need within the following technical response(s):

- A9950, Barriers for Tank/Disposal Facility Closure. Because the Subcon Focus Area infiltration barrier effort is satisfying site needs for a guidance document, the TFA did not develop a separate technical response. The TFA will revisit whether it should develop a response after Hanford has an opportunity to review the Subcon effort and submit revised needs to the TFA.

Site Need ID: RL-WT021

Site Need Title: Cleaning, Decontaminating and Upgrading Hanford Pits

Need Summary: Waste retrieved from Hanford Site tanks must pass through a number of pits associated with single-shell tanks before it is received by the privatization contractor for disposal. Many of these pits will have to be modified before the waste can be transferred. Current methods for modifying, operating, cleaning and decontaminating these pits are labor intensive, costly, and result in a high dose to workers. Currently, work associated with pits is the single largest contribution to River Protection Project (RPP) operations dose levels. For example, the dose in the 241-C-106 pits was $40 \mathrm{R} / \mathrm{hr}$. After investing $\$ 2$ million and 5 months, the dose had been reduced to only $20 \mathrm{R} / \mathrm{hr}$. During the pit operations, 25 personrems were accumulated.

Technical Disposition: The TFA responded to this need within the following technical response(s):

- A9352, Remote Systems for Pit Operations and Maintenance, TFA priority \#23.

- AA1S1, Pre-Closure Interim Tank Maintenance, TFA strategic task.

Site Need ID: RL-WT022

Site Need Title: Tank Knuckle NDE

Need Summary: Compliance to Washington Administrative Code (WAC) 173-303-640 requires life cycle integrity assessments, including non-destructive evaluation (NDE) of 6 double-shell tanks (DSTs) on a portion of the tank wall, bottom knuckle, and bottom. Additional DSTs will be selected for NDE based upon examination results. NDE equipment must be deployed to fulfill this requirement. Fracture mechanics analysis indicates that the knuckle region of the DST that rests on the concrete foundation is the highest-stressed region of the tanks. This high-stressed region is not accessible using current ultrasonic technology. This region is accessible for examination only by propagating ultrasonic energy around a plate with a one-foot radius bend. Current inspection studies demonstrate that defects in this region can be detected. However, characterizing the length and through-wall extent of defects is not possible using current technology.

Technical Disposition: The TFA responded to this need within the following technical response(s):

- A9175, Tank Integrity Inspection Techniques, TFA priority \#11.

- AA1S1, Pre-Closure Interim Tank Maintenance, TFA strategic task. 
Site Need ID: RL-WT023

Site Need Title: Prediction of Solid Phase Formation in Static and Dynamic Hanford Tank Waste Solutions

Need Summary: Information is needed on the physical and chemical properties, which represent the complex solid and liquid matrices of the Hanford Site tank waste and on the dynamics of solid phase formation during Hanford tank waste transfers. This information is needed to predict solids precipitation, gel formation, and the crystal structure of solids, which form in retrieval, wash, and leach solutions to prevent solids precipitation or gel formation during retrieval, or to recover from these phenomena. Much information is available from past solubility chemistry work at Hanford and from other DOE sites.

One part of this need is to compile available information for easier use, identify missing data, and perform work to supply the missing data. Another part of this technical need is to evaluate the dynamics of solubility in an engineering application. A pipeline needs to be evaluated to consider the dynamics of mixing and temperature effects on solubility during transfer operations. Experimentation should characterize both precipitation and redissolution kinetics so that effects of temperature and concentration changes can be identified. Attempts should be made to identify regimes where precipitation or gel formation will not occur.

Attempts should also be investigated to determine methods for recovery from precipitation/gel plugs.

The information obtained in response to this need will be used to support the development of the Hanford Office of River Protection Retrieval, Waste Feed Delivery, and Disposal Program. The Disposal Program supports the Hanford Privatization (vitrification) effort by supplying feed to a private vitrification contractor.

Technical Disposition: The TFA responded to this need within the following technical response(s):

- A9376, Waste Transfer Line Plugging Prevention and Unplugging Methods, TFA priority \#12.

- A9554, Hanford Tank Waste Chemistry, TFA priority \#4.

- AA3S1, Chemical Cleaning, TFA strategic task.

Site Need ID: RL-WT024

Site Need Title: Enhanced Sludge Washing Process Data

Need Summary: This is a continuation of the Enhanced Sludge Wash (ESW) program that has been in progress for several years. A strategy was originally developed (Kupfer 1994, Kupfer 1995) that showed how data from 47 SSTs could be used to represent $93 \%$ of the SST sludge volume. During Fiscal Years 1994, 1995, 1996, and 1997, enhanced sludge washing tests were performed on 30 samples of SST sludge to establish chemical and radionuclide removal efficiencies.

An independent review of the data available in January 1997 concluded that as much as $80 \%$ of the tank waste sludge could be processed using enhanced sludge washing, with the balance of the sludge material being treated with additional processes to meet DOE's goals on reducing glass production. Twenty percent of the tank sludge may require special handling such as selectively applied extended leach duration, or oxidative chromium leaching. From 
this review and the completion of FY 1997 testing, RL determined in September 1997 that there" is sufficient technical basis to complete the Tri-Party Agreement Interim Milestone M50-03 based on the current understanding of the tank waste compositions, tank waste pretreatment chemistry, and HLW vitrification process chemistry." (Sanders 1997).

Notwithstanding the M-50-03 determination, parts of the 1995 Kupfer sampling and testing strategy remained to be completed. The REDOX-type sludge waste contain most of the hard to remove chromium, and require additional testing to confirm chromium removal efficiencies during enhanced sludge washing and to reduce uncertainties in extrapolating data from single tanks to groups of tanks. Completion of this strategy supports retrieval sequence development and broadens the technical foundation that is needed for bidding Phase 2.

The Phase 2 ESW process is expected to be modified by performing the post-caustic-wash solid/liquid separation at an elevated temperature to improve the removal of the species of interest. The current data are based upon performing that separation at a lower temperature near ambient.

Technical Disposition: The TFA responded to this need within the following technical response(s):

- A9555, Sludge Washing and Dissolution, TFA priority \#9.

- AA3S1, Chemical Cleaning, TFA strategic task.

- AA5S1, Removal of Key Non-Radioactive Elements from Tank Waste, TFA strategic task.

Site Need ID: RL-WT026

Site Need Title: Tank Leak Detection Systems for Underground Single-Shell Waste Storage Tanks (SSTs)

Need Summary: The use of past-practice sluicing for SST waste removal involves the addition of liquid to tanks and therefore increases the potential for waste leakage to the environment. Leak detection applies to all SST retrieval, including retrieval during Phase I and preparation of the Phase II specification. Leak detection methods are needed that can signal and quantify a leak from a tank when only a small amount of waste has been released. Technical Disposition: The TFA responded to this need within the following technical response(s):

- A9156, Tank Leak Detection, TFA priority \#33.

Site Need ID: RL-WT027

Site Need Title: Tank Leak Mitigation Systems

Need Summary: The use of liquid based retrieval methods for SST waste removal involves the addition of liquid to tanks and therefore increases the potential for waste leakage to the environment. Leakage mitigation applies to all SST retrieval, including retrieval during Phase I and preparation of the Phase II specification. Leakage mitigation efforts and tools that can be shown to provide cost-benefit and significant risk reduction over baseline methods should be incorporated into retrieval system design and operating procedures. Existing mitigation techniques (i.e., the current baseline approach) must continue to be evaluated against potential/candidate mitigating technologies to ensure that the most costeffective, risk reducing approach is applied. Periodic identification and evaluation of 
potential leakage mitigation tools for possible application during SST retrieval operations is required on a continuing basis.

Technical Disposition: The TFA responded to this need within the following technical response(s):

- A9157, Tank Leak Mitigation, TFA priority \#20.

- AA1S1, Pre-Closure Interim Tank Maintenance, TFA strategic task.

- AA3S2, SST Retrieval from Potential Leaking Tanks, TFA strategic task.

Site Need ID: RL-WT029

Site Need Title: Data and Tools for Performance Assessments

Need Summary: Performance assessments must be developed for all disposal actions. The models that are used for these assessments require a defensible basis for the movement of water. Most databases describe recharge and distribution of water for non-arid conditions. The arid conditions at the Hanford Site are not accurately represented by the existing data. This need is comprised of two elements:

- Recharge water is the primary means for dissolution and release of contaminants from the buried waste and transport of those contaminants to the groundwater. Estimation of these rates is difficult under arid conditions because the rates are very low. In addition, there are significant questions about the adequacy of the estimated recharge rates given the heterogeneity of the environmental processes, the effect of facility features, the uncertainty of climate, and the influence of humans. Furthermore, no attempt has been made to quantify the distribution of recharge rates to enable sounder estimates of the mean and range of rates to be expected during the time of compliance of the facility.

Assessments of waste disposal require the knowledge of hydraulic properties in the unsaturated sediments (the vadose zone). Typically, these properties are inferred or estimated from small cores or particle size distributions obtained from a drilled borehole. Field measurements of hydraulic properties will eliminate the uncertainty when extrapolating small-scale laboratory measurements. (This technology needs statement has been included in the Subsurface Contaminants needs list.)

Technical Disposition: The TFA responded to this need within the following technical response(s):

- A9958, Data and Tools for Performance Assessments. In discussions with Hanford site personnel, the TFA has learned that the objectives of this technical response are being pursued through the incorporation of these technology needs in the Groundwater/Vadose Zone program. Therefore, the TFA will not prepare a full technical response to this need.

Site Need ID: RL-WT060

Site Need Title: Better Waste Mixing Mobilization

Need Summary: 1) Hanford needs enhanced sludge mobilization methods to retrieve sludge that is beyond the Effective Cleaning Radius (ECR) of the baseline pair of long-shaft mixer pumps. The objective is a small system that can be installed in the tanks along with the mixers when needed to mobilize the remaining sludge.

2) Both Hanford and SRS are interested in identifying replacements for baseline mixer pumps with more cost-effective alternates with respect to life-cycle/operations costs for bulk 
sludge, sludge heel, and salt cake retrieval, both in large HLW storage tanks and in smaller process tanks such as SRS transfer system pump tanks. Safety impacts to Authorization Bases also need to be evaluated. The TFA is evaluating the use of Flygt mixers for SRS this year as part of this goal.

3) Hanford needs mixer pumps which can start at very low RPM in very viscous (one million + centipoise) waste to de-gas tanks.

4) Hanford needs mixer pumps which can remove the pumping energy (i.e., cooling), added to the tank to avoid waste heating.

Technical Disposition: The TFA responded to this need within the following technical response(s):

- A9359, Waste Mixing and Retrieval, TFA priority \#34.

- AA3S1, Chemical Cleaning, TFA strategic task.

Site Need ID: RL-WT061

Site Need Title: Reactive Barriers to Contaminant Migration

Need Summary: Although the SSTs and DSTs store a broad range of highly radioactive isotopes, a few relatively mobile constituents dominate the risk to human health and the environment. Based on past analysis, for the vadose zone groundwater pathway, the list typically includes carbon-14, technetium-99, iodine-129, selenium-79 and uranium. The relative importance of these constituents may vary depending on assumptions used during the specific analysis. Retrieval of waste from these tanks will incur risk from additional leakage. If these key radioactive elements could be trapped or immobilized in the waste matrix, disposal facility, closed tanks, and/or the soil column, the risk to human health and the environment could be significantly reduced. It is proposed that sequestering agents be deployed as a permeable flow-through (reactive) barrier to attenuate the migration of these contaminants and reduce the risk.

It is proposed that sequestering agents be deployed as a permeable flow-through (reactive) barrier to attenuate the migration of these contaminants and reduce the risk. In the case of contaminated soil, the reactive barrier will be placed using conventional emplacement technology, e.g., slant drilling, etc. For the vitrified waste and for tank closure, it is proposed that the getter could be placed inside the facility.

Technical Disposition: The TFA responded to this need within the following technical response(s):

- A9960, Sequestering of Contaminant Migration, TFA priority \#36.

Site Need ID: RL-WT062

Site Need Title: Variable Suction Level Transfer Pump

Need Summary:

All DSTs containing insoluble solids need a variable level suction transfer pump. The current baseline transfer pump achieves variable suction levels by using a flexible hose controlled by a tether cable. This design can't be operated simultaneously with mixer pumps because of hose instability. A transfer pump that can draw waste from pre-selected levels that range from the surface to within $10^{\prime \prime}$ of the tank bottom is needed. 
Technical Disposition: The TFA responded to this need within the following technical response(s):

- A9365, Waste Transfer Pumping, TFA priority \#8.

Site Need ID: RL-WT063

Site Need Title: PHMC Retrieval and Closure - Hanford SST Saltcake Dissolution Retrieval Need Summary: Performance data and retrieval efficiency data are required for a simplified sprinkler-applied (low volume) water dissolution system for use in Hanford's SSTs contianing saltcake waste. Effects of in-tank hardware and tank walls shall also be determined. This system is also known as the Low Volume Density Gradient (LVDG) retrieval method. Application of this method to a representative stimulant of waste shall provide the necessary data to select this method for baseline implementation.

Technical Disposition: The TFA responded to this need within the following technical response(s):

- A9362, Salt Cake Dissolution Retrieval, TFA priority \#19.

- A9554, Hanford Tank Waste Chemistry, TFA priority \#4.

- AA3S1, Chemical Cleaning, TFA strategic task.

- AA3S2, SST Retrieval from Potential Leaking Tanks.

Site Need ID: RL-WT064

Site Need Title: PHMC Retrieval and Closure - Hanford Past Practice Sluicing Improvements

Need Summary: Improvements in sluicing technology have been made since past practice sluicing was performed at Hanford for tank waste retrieval. A better understanding of these improvements and how they compare to past practice sluicing is needed to optimize waste retrieval operations. A direct comparison between the past practice sluice nozzles and current industrial nozzles capabilities needs to be performed to provide the most effective design requirements to support high-level waste (HLW) feed delivery. The comparisons must provide a clear quantitative analysis of the ability of each nozzle and pump type and configuration and its ability to move different waste types.

Technical Disposition: The TFA responded to this need within the following technical response(s):

- A9367, Unobstructed Tank Heel Retrieval, TFA priority \#26.

- AA3S1, Chemical Cleaning, TFA strategic task.

- AA3S2, SST Retrieval from Potential Leaking Tanks.

Site Need ID: RL-WT065

Site Need Title: Direct Inorganic and Organic Analyses of High-Level Waste.

Need Summary: Characterization is required for waste feed certification and acceptance, payment for services and qualification of secondary waste, intermediate, and vitrified products. Characterization of HLW is a key part of certifying that the tank waste composition and inventory meets the minimum reporting requirements for selected constituents in the HLW feed samples as specified in the RPP (formerly TWRS) Privatization Contract (DEAC06-96-RL13308, Mod A005). Waste acceptance criteria developed through the treatment facility Dangerous Waste Permit Application will include characterization needed to ensure regulatory compliant treatment, storage, and disposal of the waste, including requirements for 
meeting land disposal restrictions, delisting, and permitting of the treatment facility. Waste products will be characterized to demonstrate compliance with disposal facility waste analysis plans. Characterization to support waste processing will be applied during a number of steps in the treatment cycle, including waste storage, staged feed, treatment, waste products qualification and disposal. A direct chemical analysis of tank waste inorganic and organic constituents would reduce chemical anaysis turn-around time, waste production, worker exposure and the potential for high cost impacts resulting from delays in feed delivery (idle facility fees are estimated as high as $\$ 250 \mathrm{M}$ per feed batch). Deployment of technology(ies) within the next two years can significantly improve key elements of the ORP RPP permitting and feed staging baseline activities.

Technical Disposition: The TFA responded to this need within the following technical response(s):

- A9264, Improve Waste Analytical Methods, TFA priority \#37.

Site Need ID: RL-WT066

Site Need Title: Compositional Dependence of the Long Term Performance of Glass as a Low-Activity Waste Form

Need Summary: Present plans for the 54 million gallons of Hanford tank waste are to retrieve the waste from the underground tanks, separate the waste into a high-level fraction (containing most of the radionuclides and hazardous materials) and a low-activity fraction (containing most of the waste volume). Both fractions will be immobilized, with the immobilized high-level fraction stored on-site until shipped to a federal geologic repository and the immobilized low-activity fraction disposed of on the Hanford Site.

Because of the relatively large amount of contaminants in the ILAW form, the rate of release must be slow and the rate limited for hundreds of thousands of years. Estimating such a longterm release rate from short-term experiments (even those lasting many years) requires a strong database, an understanding of the degradation process, and numerical simulation tools that combine the database and a mathematical model of the glass corrosion process.

ORPs Immobilized Waste Program is performing a series of tests on representative LAW glasses to better understand how likely glasses will perform over these long periods of time. The vision for this work is given in "A Strategy to Conduct an Analysis of the Long-Term Performance of Low-Activity Waste Glass in a Shallow Subsurface Disposal System at Hanford," (PNNL 18834, or Appendix G of DOE/RL-97-69). However, there are some areas presently not being funded by EM-30. Rather the support is from EM-50, because of its greater applicability to other potential disposal actions. In particular, the database must be expanded so the affect of different glass compositions on long-term performance can be determined. An important subset of this need is to understand how glass composition impacts the rate of sodium ion-exchange in LAW glasses, which has been found to significantly affect the calculated $\mathrm{pH}$ in the disposal system and thus the long-term radionuclide release rate. 
Technical Disposition: The TFA responded to this need within the following technical response(s):

- A9748, Testing and Prediction of Long-Term Waste Glass Performance, TFA priority \#25.

- AA7S1, Durability of Multiphase Waste Glasses

Site Need ID: RL-WT067

Site Need Title: Improved DST Integrity NDE Measurement Tools

Need Summary: Compliance to WAC 173-303-640 requires life cycle integrity assessments, including non-destructive examination (NDE) of 6 DSTs on a portion of the tank wall, bottom knuckle, and bottom. Additional DSTs will likely be selected for NDE based upon examination results. The size of the current NDE equipment restricts its deployment access to two 24-inch risers, diametrically opposed, per DST. NDE of the DSTs is also limited to 20 to $25 \%$ of the tank circumference, in the regions closest to the 24 -inch risers. NDE equipment that could be deployed through smaller diameter risers would provide access to a larger percentage, potentially all, of the tank circumference.

Technical Disposition: The TFA responded to this need within the following technical response(s):

- A9175, Tank Integrity Inspection Techniques, TFA priority \#11.

- AA1S1, Pre-Closure Interim Tank Maintenance

Site Need ID: RL-WT068

Site Need Title: Radionuclide Source. Term from Tank Residuals

Need Summary: Need Description: Remediation and closure of HLW tanks at Hanford will leave residual solids that are estimated to be one of the major long-term radionuclide sources into underlying vadose zone sediments. However, the actual release rate of technetium, selenium, and uranium (the major predicted dose contributors) from the residuals is essentially unknown. A fundamental understanding of the true radionuclide source-term from the residuals is needed to base sound cost/benefit/risk decisions regarding the extent of waste removal actually required from the tanks to meet site-wide groundwater protection standards. Technical Disposition: The TFA responded to this need within the following technical response(s):

- A9588, Leaching and Treatment of Technetium for Tank Closure, TFA priority \#41.

Site Need ID: RL-WT069

Site Need Title: Value of Information Decision Analysis for Tank Farm Closure Need Summary: The purpose of waste retrieval from SSTs is to prepare tanks for closure. The Environmental Impact Statement (EIS) for the RPP (formerly Tank Waste Remediation System) evaluated waste retrieval and disposal alternatives, but did not evaluate tank farm closure alternatives because sufficient information was not available. The Record of Decision for the EIS committed to conducting a NEPA process for decisions on tank farm closure when sufficient additional information was available on waste retrieval performance, closure technology, and vadose zone conditions. Until decisions on closing tank farms are made, final requirements for SST waste retrieval cannot be specified. This includes requirements for allowable residual waste in SSTs following completion of retrieval. Unless a default 
value of zero leakage during retrieval is specified as an interim requirement, final requirements for leakage control during retrieval also are constrained by decisions on how tank farms will be closed, since closure decisions include decisions on remediation of soil potentially contaminated by retrieval leaks. Tri-Party Agreement milestones for characterizing the vadose zone in SST waste management areas (WMAs) under RCRA assessment have been established.

Phase 1 of the required investigations are scheduled to be completed in FY 2004, with recommendations for additional subsurface investigations that may be required to support decisions on waste retrieval and closure. Presently, criteria for making closure decisions (é.g., quantitative measure(s) of compliance, points of compliance, period of compliance, exposure scenarios) have not been established, although criteria are required to be established under the recently adopted Tri-Party Agreement milestones. Also, no criteria have been established to guide the decision due in FY 2004 on whether additional subsurface data may be needed in a second phase of subsurface investigations in the SST WMAs under RCRA assessment. Because subsurface investigations in contaminated tank farm soils are expensive, an approach is needed for determining when enough information has been gathered to support decisions on closure.

Technical Disposition: The TFA responded to this need within the following technical response(s):

- A9924, Tank Closure Criteria/Decision Support, TFA priority \#30.

Site Need ID: RL-WT070

Site Need Title: Uncertainty Estimation of Hanford Best Basis Toxic Waste Inventory, Concentration, Phase and Waste Type

Need Summary: Hanford currently has static single value point estimates, best basis inventory estimates, and associated initial estimates of the uncertainty of those point estimates, for 71 analytes in each of 177 tanks. This is not sufficient to support the "dynamic" nature that the tank farms currently exhibit, let alone the increased waste transfer activity that will occur during retrieval and treatment processing. Additionally, current estimates do not relate information about phase (liquid, saltcake, sludge, hardpan) location of each waste type within the tank, or concentrations at each location in each tank. This does not support a clear understanding of what type of waste will be retrieved from a tank at the beginning, middle, or end of the retrieval process for a single tank. Because most tanks have multiple waste phases and/or waste types located in layers (roughly speaking) and retrieval efforts will generally retrieve part of the waste in a tank (or parts of multiple tanks), use of "tank average" estimates are likely to be seriously inaccurate for any given retrieval effort.

The retrieval process will be guided by the need to contribute waste to the staging tank(s) to assure the mixed waste feed transferred to the privatization contractor is within the required processing envelope and is an efficient mixture from the government's point of view (minimum storage volume and minimum sodium added). Additionally, it will be necessary to ensure that the tank(s) contributing the waste and the tank(s) receiving waste are safe throughout the process. In order to do this, we must know the waste characteristics (chemical and phase) and their locations in the tank so we can know what is retrieved (and what remains) throughout the retrieval process of each individual tank. 
Finally, the uncertainty associated with each estimated value must be predicted. This will allow for waste retrieval management with appropriate cushions for error and reduce the risk to a minimal level.

Technical Disposition: The TFA responded to this need within the following technical response(s):

- A9555, Sludge Washing and Dissolution, TFA priority \#9.

- AA3S1, Chemical Cleaning, TFA strategic task.

- AA5S1, Removal of Key Non-Radioactive Elements from Tank Waste

Site Need ID: RL-WT071

Site Need Title: Provide Laboratory Development Support and ESP Modeling Support for the Back Dilution of Tank 241-SY-101

Need Summary: The waste in SY-101 will be diluted during transfer; then added to waste in SY-102; and back diluted when free board is available. These dilutions use varying amounts of water or other waste. There are too many options to test all of the options in the laboratory. The current intent is to perform enough laboratory experiments to give a range of dilutions but use the Environmental Simulation Program (ESP) model to interpolate or extrapolate that information to other cases. This will be unique to SY-101 waste, but will help to demonstrate the utility of using ESP for other waste problems.

Technical Disposition: The TFA responded to this need within the following technical response(s):

- A9554, Hanford Tank Waste Chemistry, TFA priority \#4.

- AA3S1, Chemical Cleaning, TFA strategic task.

Site Need ID: RL-WT072

Site Need Title: Use of Handheld Technology to Automate Operator Data Sheets for Tank Farm Operations

Need Summary: Hanford Tank Farm Operations desires the ability to improve the operator rounds function. Conduct of Operations improvements are needed to automate data collection from the field in order to optimize the Shift Manager's ability to analyze plant conditions that enable him to make informed decisions based on real time data, trends, alarms, etc.

Technical Disposition: The TFA responded to this need within the following technical response(s):

- AA101, Automated Data Collection System for Tank Operations. Meetings were held with the technical points of contact as well as with the chief information officer for CH2M Hill Hanford Group (CHG). In addition, preliminary research into the availability of automated, bar code reader-based data collection systems was conducted. As the result of these meetings and research, the Hanford Site has acknowledged that it is premature to move forward in addressing this need.

Site Need ID: RL-WT080

Site Need Title: Advanced/Improved Vitrification

Need Summary: Includes higher temperature joule heated melters, cold wall or cold crucible melters, and higher waste loading techniques (i.e., dealing with problem constituents). 
Technical Disposition: The TFA responded to this need within the following technical response(s):

- A9768, Specify and Enhance Design of HLW Glass Melters, TFA priority \#3.

- A9773, Improve Waste Loading in HLW Glass, TFA priority \#5.

- AA7S1, Durability of Multiphase Waste Glasses, TFA strategic task.

- AA7S2, New Melter Technology, TFA strategic task.

Site Need ID: RL-WT081

Site Need Title: Sulfate Accumulation in Low Activity Waste

Need Summary: Includes pretreatment, vitrification, and offgas approaches to sulfate management.

Technical Disposition: The TFA responded to this need within the following technical response(s):

- A9773, Improve Waste Loading in HLW Glass, TFA priority \#5.

- AA7S1, Durability of Multiphase Waste Glasses, TFA strategic task.

Site Need ID: RL-WT082

Site Need Title: Crystalline Silicotitanate Non-Elutable Sorbent

Need Summary: Advanced Cs separations for pretreatment. Limited scope to evaluate specific Hanford waste streams.

Technical Disposition: The TFA responded to this need within the following technical response(s):

- A9570, Salt Disposition, TFA priority \#6.

Site Need ID: RL-WT083

Site Need Title: Rapid PCB Screening Technology

Need Summary: As a result of the extensive use of polychlorinated biphenyl (PBC)containing products, contamination levels must be determined at nearly all facilities throughout the DOE complex, including the RPP's HLW storage tanks at Hanford. Standard methods of laboratory analysis require several days to complete. A rapid quantitative field screening product is needed that will streamline analytical laboratory measurements by identifying and approximating the concentration of PCBs present in tank waste samples at the regulated concentration thresholds. Scoping evaluation with a potential developer has confirmed the viability of developing the product.

Technical Disposition: The TFA responded to this need within the following technical response(s):

- A9264, Improve Waste Analytical Methods, TFA priority \#37.

Site Need ID: RL-WT084

Site Need Title: Extension of Glass Properties Model to Lower Silica Compositions Need Summary: The "Glass Properties Model" developed by PNNL must be extended to provide reliable predictions of glass liquidus temperature for glass compositions containing as little as $30 \%$ silica.

Technical Disposition: The TFA responded to this need within the following technical response(s):

- A9773, Improve Waste Loading in HLW Glass, TFA priority \#5. 
Site Need ID: RL-WT085

Site Need Title: Retrieval of Waste Heel from 340 Radioactive Liquid (Low-Level / Mixed Waste Vault) Vault Tanks

Need Summary: Until 1998, the 340 Facility received liquids wastes from a variety of sources in Hanford's 300 Area, then transferred the liquids to a railroad tanker for transport to the 200 Area Tank Farms. In 1998, the Hanford railroad network was shut down, as were the 340 waste receiving and transfer operations. As part of the initial 340 Facility deactivation activities in 1998, the tanks were pumped to remove all but the last/lowest 24 inches of liquids and sludge. This 24 -inch waste level represents the lowest elevation of the in-tank pump inlet.

A method is needed for removing residual waste from two 15,000 gallon tanks. The tanks are situated below grade in a concrete vault. Waste remaining in each tank is comprised of about 1,500 gallons of liquids, sludge, solids and dispersible materials. Tank internals that may challenge the cleanout effort include a non-functional, four-paddle, 4-feet diameter, agitator located near the bottom of the tank. The tanks also have three, concentrically spaced, vertical support baffles in the interior knuckle region of the tank (likely regions of sludge "hold-up"). Technical Disposition: The TFA responded to this need within the following technical response(s):

- A9382, Horizontal and Small Tank Sludge Mixing and Retrieval, TFA priority \#27.

Site Need ID: RL-WT031-S

Site Need Title: Rapid Waste Characterization

Need Summary: Characterization of tank waste is needed to: 1) support methods used to determine what, if any, actions are required to assure safe interim storage of each waste type;

2) determine waste physical properties to assist in development of transport methods and requirements; 3 ) certify that the composition and inventory meets the minimum reporting requirements for selected constituents in feed samples as specified in the RPP Privatization Contract (DE-AC06-96-RL13308, Mod A005), and 4) support the basis for determining payment of waste treatment services by the private contractor (BNFL, Inc.). A method of providing rapid in-situ (either within tanks or in-line) characterization of chemical and radioactive constituents could reduce the turn around time to provide process control or composition information, as well as reduce the number of samples required.

Qualification of the absence of a separable organic layer in Hanford tank waste destined to the private contractor is needed to meet contractual requirements. Rapid yes/no evaluation of a separable organic layer is needed to assure adequate storage, retrieval and transfer. Information concerning the chemical forms and concentrations of the chemical and radioactive constituents of waste is necessary before mixing of waste to ensure compatibility of the waste considered for mixing.

Under the RPP Privatization Contract, the private contractor operating waste treatment facilities will be paid for services based on a combination of the amount of sodium processed, the waste oxide loading in the immobilized waste, and the number of units of waste produced. In particular, a determination of soluble sodium (vs. insoluble sodium in 
entrained solids) delivered with the feed will be required. Accurate, rapid characterization of the feed stream would enhance the feed certification activities by providing timely information for certifying tank composition and supporting the determination of payment for waste treatment services.

Technical Disposition: The TFA responded to this need within the following technical response(s):

- AA202, In-Situ Waste Characterization, TFA priority \#32.

Site Need ID: RL-WT032-S

Site Need Title: Monitoring of Key Waste Physical Properties During Retrieval and Transport

Need Summary: Monitoring of key waste physical properties during retrieval and transport of the material between tanks and to the privatization contractor is needed to meet the minimum physical property requirements for LAW feed and HLW feed as specified in the RPP Privatization Contract (DE-AC06-96-RL13308, Mod A005).

To meet contractual requirements, it is necessary for LAW transferred to the private contractor to contain less than 2 weight percent (dry basis) solids. For HLW transferred to the private contractor, it is desirous to transfer the solid material to the private contractor, and contractually required to transfer waste to the private contractor with at least 10 grams of unwashed solids per liter of solution and up to $200 \mathrm{~g} / \mathrm{L}$.

Technical Disposition: The TFA responded to this need within the following technical response(s):

- A9278, Slurry Transfer and Tank Waste Mixing Monitors, TFA priority \#35.

Site Need ID: RL-WT035-S

Site Need Title: Moisture Flow and Contaminant Transport in Arid Conditions Need Summary: To understand the movement of contaminants through zones of low moisture (region-wide saturation less than 10\%) for use in risk assessments.

Technical Disposition: The TFA responded to this need within the following technical response(s):

- A9958, Data and Tools for Performance Assessments. In discussions with Hanford Site personnel, the TFA has learned that the objectives of this technical response are being pursued through the incorporation of these technology needs in the Groundwater/Vadose Zone program. Therefore, the TFA will not prepare a full technical response to this need.

Site Need ID: RL-WT037-S

Site Need Title: Sludge Treatment

Need Summary: Sludge leaching with concentrated $\mathrm{NaOH}$ solutions at elevated temperatures is the proposed strategy for the removal of $\mathrm{Al}$ and $\mathrm{Cr}$ from the RPP waste stream. Systematic evaluations of the effects of temperature, alkalinity, ionic strength and other parameters on the rates of dissolution and solid state phase transformations are presently unavailable. Our present level of understanding of the behavior of $\mathrm{Cr}$ in the Hanford waste tanks is inadequate. There are few available data on the equilibrium behavior of $\mathrm{Cr}$ compounds in tank-like environments, and kinetic information under these conditions 
is virtually nonexistent. Since available data from tank sludge samples indicates that chromium in the solid phases is present mostly as $\mathrm{Cr}$ (III) whereas, in the aqueous phase, $\mathrm{Cr}$ appears to be present mostly as $\mathrm{Cr}(\mathrm{VI})$, fundamental investigations of the equilibria and kinetics of reactions involving the $\mathrm{Cr}(\mathrm{III}) \mathrm{S} \mathrm{Cr}$ (VI)aq transitions are necessary. Furthermore, such transitions are likely to be strongly dependent on temperature, alkalinity and various other parameters. Thus, a systematic investigation of the general equilibria and dissolution/precipitation kinetics of $\mathrm{Cr}$ compounds in concentrated alkaline solutions is key to predicting the behavior and speciation of $\mathrm{Cr}$ in the Hanford tank systems.

Technical Disposition: The TFA responded to this need within the following technical response(s):

- A9555, Sludge Washing and Dissolution, TFA priority \#9.

- AA3S1, Chemical Cleaning, TFA strategic task.

- AA5S1, Removal of Key Non-Radioactive Elements from Tank Waste, TFA strategic task.

Site Need ID: RL-WT038-S

Site Need Title: Process Models for Sludge Treatment

Need Summary: Information is needed on the solubility of various components in the complex solid and liquid matrices of the Hanford tank waste, especially those associated with sludges. This information is needed to predict when solids will precipitate or when gels will form in retrieval, wash, and leach solutions, and to supplement empirical water wash and caustic leach data from enhanced sludge wash testing of Hanford tank sludge samples and other data from dissolution testing of saltcake samples.

Predicting the precipitation of solids in a complex, concentrated brine requires a suitable model and a well-designed set of data from which model parameters can be obtained. Although the identity and approximate abundance of major and minor chemical components in the Hanford tanks are fairly well defined, there are inadequate fundamental experimental data to support an adequate predictive model, and there has been inadequate use of existing data. The solubilities of solid phases in high-ionic strength brines that approximate subsets of the actual Hanford chemical systems need to be measured to: a) determine equilibrium constants, and b) extract electrolyte model parameters describing the behavior of sparingly soluble compounds.

An understanding of the behavior of the solutions produced by the enhanced sludge wash (ESW) process and their interactions with other waste in the Hanford tanks is needed to prepare for Phase 1 and Phase 2 privatization and for evaluation of vendors' proposals. The scope of this work is complementary to work performed under need statement RL-WT037-S and supports technology need statement RL-WT024.

Technical Disposition: The TFA responded to this need within the following technical response(s):

- A9555, Sludge Washing and Dissolution, TFA priority \#9.

- AA3S1, Chemical Cleaning, TFA strategic task.

- AA5S1, Removal of Key Non-Radioactive Elements from Tank Waste, TFA strategic task. 
Site Need ID: RL-WT040-S

Site Need Title: Mechanisms of Line Plugging

Need Summary: Inter-area transport lines for particulate slurries have plugged in the past due to particle settling, phase changes, or reactions accompanied by precipitation or gel formation that occurred during transport. Information to predict pressure drop and critical transport velocity of waste with known properties is required to ensure that waste can be safely transported without risk of plugging. To minimize the dilution required to modify waste properties, methods to predict the effect of dilution, washing, or leaching on the slurry properties is also required. Dilution both increases the volume of the waste and has negative implications for tank waste management both from a space perspective and for settling and separation of solids. Waste compatibility is also an issue in the case of waste blending from several simultaneous or sequential retrieval campaigns.

Technical Disposition: The TFA responded to this need within the following technical response(s):

- A9376, Waste Transfer Line Plugging Prevention and Unplugging Methods, TFA priority \#12.

- A9554, Hanford Tank Waste Chemistry, TFA priority \#4.

- AA3S1, Chemical Cleaning, TFA strategic task.

Site Need ID: RL-WT043-S

Site Need Title: Effect of Human and Natural Influences on Long-Term Water Distribution Need Summary: Fundamental data are needed to improve confidence in performance assessment under realistic conditions. Efforts are needed to 1) consider long-term land and water use at DOE sites by future generations; 2) consider natural phenomena such as nearterm climate change (which is forecast to impact society in the next 100 years) or long-term climate change as we transition into the next ice age; and 3) incorporate those uses and impacts into modeling efforts to predict the transport of contaminants.

Technical Disposition: The TFA responded to this need within the following technical response(s):

- A9958, Data and Tools for Performance Assessments. In discussions with Hanford Site personnel, the TFA has learned that the objectives of this technical response are being pursued through the incorporation of these technology needs through the Groundwater/Vadose Zone program. Therefore, the TFA will not prepare a full technical response to this need.

Site Need ID: RL-WT044-S

Site Need Title: Distribution of Recharge Rates

Need Summary: Fundamental data is needed to improve confidence in performance assessments under realistic conditions.

Technical Disposition: The TFA responded to this need within the following technical response(s):

- A9958, Data and Tools for Performance Assessments. In discussions with Hanford Site personnel, the TFA has learned that the objectives of this technical response are being pursued through the incorporation of these technology needs in the Groundwater/Vadose Zone program. Therefore, the TFA will not prepare a full technical response to this need. 
Site Need ID: RL-WT045-S

Site Need Title: Vadose Zone Flow Simulation Tool Under Arid Conditions

Need Summary: An understanding of the movement of contaminants in very complex geometries through zones of low moisture for use in risk assessments is needed.

Technical Disposition: The TFA responded to this need within the following technical response(s):

- A9958, Data and Tools for Performance Assessments. In discussions with Hanford Site personnel, the TFA has learned that the objectives of this technical response are being pursued through the incorporation of these technology needs in the Groundwater/Vadose Zone program. Therefore, the TFA will not prepare a full technical response to this need.

Site Need ID: RL-WT046-S

Site Need Title: Getter Materials

Need Summary: Fundamental data is needed to improve confidence in performance assessments under realistic conditions. In order to meet the contaminant release specifications for the disposal of Hanford LAW, radiocontaminants are physically trapped in glass. However, only a few of these radioelements drive the performance assessment. If these key radioelements could be chemically trapped after their release from glass, then the performance of the waste disposal system could be significantly improved. Hydraulic properties of getter materials (original, loaded, and discharged) need to be measured to fully understand waste disposal performance in the presence of getters. The use of getter materials in the Savannah River Site's disposal of the Saltstone waste was an important consideration in the approval of that site's disposal of tank waste.

Technical Disposition: The TFA responded to this need within the following technical response(s):

- A9960, Sequestering of Contaminant Migration, TFA priority \#36.

Site Need ID: RL-WT049-S

Site Need Title: Effect of Processing on Gas Release, Waste Sedimentation, Rheological, and Other Behaviors

Need Summary: This is a consolidation of FY 1999 RL-WT049-S, Effect of Processing on Waste Rheological and Sedimentation Properties, and RL-WT051-S, Foam Generation and Stability. Current process concepts assume that mixer pumps are effective at degassing tanks and that mixer pumps have no detrimental impact on sedimentation, rheological properties, and other behaviors of the Hanford tank waste. The potential for foaming from retained gases and inadvertent precipitation reactions from mixing has not been evaluated.

Technical Disposition: The TFA responded to this need within the following technical response(s):

- A9554, Hanford Tank Waste Chemistry, TFA priority \#4.

- AA3S1, Chemical Cleaning, TFA strategic task.

Site Need ID: RL-WT052-S

Site Need Title: Characterization of Organic Species in Waste Feed to LAW and HLW Treatment Facilities 
Need Summary: Development of analytical methods are needed to address DOE and regulatory requirements for organic species in Hanford tank waste. The analyses could use existing analytical tools but would require the development of sample preparation steps, calibration, and method validation for their application to organic species in tank waste. Measurement is needed of the amount of certain RCRA and Toxic Substances Control Act of 1976 (TSCA) organic compounds in waste feed to the private contractors that may impact process and plant design. Since the waste is a very complex matrix, analytical methods must be modified for suitable application. Some of this method modification has been done at the Hanford 222-S Laboratory. Future efforts should be coordinated with the work already done to avoid duplication.

Technical Disposition: The TFA responded to this need within the following technical response(s):

- A9264, Improve Waste Analytical Methods, TFA priority \#37.

Site Need ID: RL-WT054-S

Site Need Title: Solids Yield During Mixer Pump Mobilization

Need Summary: Validated mixer pump performance correlations, (i.e., effective cleaning radius [ECR]) as a function of definable properties.

Technical Disposition: The TFA responded to this need within the following technical response(s):

- A9359, Waste Mixing and Retrieval, TFA priority \#34.

Site Need ID: RL-WT056-S

Site Need Title: Half-Lives of Se-79 and Sn-126

Need Summary: Immobilized waste will be disposed of starting in 2002. Measurements of the half-lives of Se-79 and Sn-126 are needed to within $+/-10 \%$ to determine if additional separations are needed, and if special operational handling is necessary.

Technical Disposition: The TFA responded to this need within the following technical response(s):

- A9958, Data and Tools for Performance Assessments. In discussions with Hanford Site personnel, the TFA has learned that the objectives of this technical response are being pursued through the incorporation of these technology needs in the Groundwater/Vadose Zone program. Therefore, the TFA will not prepare a full technical response to this need.

Site Need ID: RL-WT075-S

Site Need Title: HLW Solid Phase Characterization

Need Summary: Provide tank waste solid phase analytical identification capability and routine waste solid phase characterization to give the following:

- early detection of problem tank waste components

- accurate prediction of solid and solution phase partitioning for best basis inventory

estimates

- technical bases for blending, dilution, and RPP retrieval operations

- input to sludge washing flowsheet parameter testing and operations

- guidance for optimized vitrification feed composition. 
Technical Disposition: The TFA responded to this need within the following technical response(s):

- A9554, Hanford Tank Waste Chemistry, TFA priority \#4.

- AA3S1, Chemical Cleaning, TFA strategic task.

Site Need ID: RL-WT077-S

Site Need Title: Improvements to Salt Well Pumping

Need Summary: Improvements in the speed and completeness of salt well pumping are required to enhance the stabilization of SSTs. Safe and practicable methods are required to enhance the drainage and speed and completeness of removal of liquor through salt well pumping. These methods also have potential utility in the ultimate sluicing and removal of waste from both the SSTs and DSTs in future D\&D operations.

Technical Disposition: The TFA responded to this need within the following technical response(s):

- A9362, Salt Cake Dissolution Retrieval, TFA priority \#19.

- AA3S2, SST Retrieval from Potential Leaking Tanks, TFA strategic task.

Site Need ID: RL-WT078-S

Site Need Title: Plutonium Segregation and Association in HLW

Need Summary: Tests with genuine waste sludges and solutions are required to determine the disposition of plutonium to sludge solid phases according to solids particle size, composition, and sedimentation rate and to determine if plutonium can segregate from neutron poisons present in HLW solid phases by physical or chemical mechanisms envisioned in RPP operations. Such mechanisms include sluicing, settling, and chemical leaching by organic complexants, carbonate, aluminate, and hydroxide. The analytical results must be interpreted to identify correlations of plutonium concentration to solids settling velocity and the concentrations of the major chemical elements in light of their neutron poisoning capacity.

Technical Disposition: The TFA responded to this need within the following technical response(s):

- A9554, Hanford Tank Waste Chemistry, TFA priority \#4.

- A9555, Sludge Washing and Dissolution, TFA priority \#9.

- AA3S1, Chemical Cleaning, TFA strategic task.

- AA5S1, Removal of Key Non-Radioactive Elements from Tank Waste, TFA strategic task.

Site Need ID: RL-WT079-S

Site Need Title: Double Shell Tanks Corrosion Chemistry

Need Summary: In FY 1999, DST 241-AN-105 was discovered to have wall thinning significantly in excess of predictions from uniform corrosion rate estimations. Although some theories have been put forward, the exact cause of this wall thinning is still unknown. The waste chemistry in this tank is within the Hanford Site's operating limits for corrosion control but may not be providing the expected protection. Plans are to ultrasonically reexamine the tank within the next five years to assure the corroded regions are not thinning at a rate inconsistent with the waste specifications. Previous studies by PNNL have identified dilute waste chemistries promoting excessive corrosion attack, and more concentrated wastes 
producing high corrosion rates at temperatures exceeding the DST operating temperature range. Future tank waste operations are expected to process wastes that are more dilute with respect to some corrosion inhibiting waste constituents.

This need calls for the implementation of a two-year laboratory corrosion evaluation of simulated (non-radioactive) waste with chemistries and temperatures bounding the variations of chemistry and temperature in tank 241-AN-105 and bridging to dilute waste chemistries expected in the future. The specific purpose of the study will be to identify waste chemistries and temperatures within the DST operating limits for corrosion control and operating temperature range which may not provide the expected corrosion protection, and evaluate future operations for the conditions outside the existing corrosion coupon database. This corrosion control issue will affect all of the dilute waste storage tanks at Hanford, and may ultimately impact all 28 DSTs. This need supports TWRS Program Logic "Conduct Tank Farms Safe Operations" and "Conduct Reduced Mortgage Tank Farm Safe Operations." Corrosion control is discussed in the Safe Storage Technical Basis Report, Activity Number 190.N45.

Technical Disposition: The TFA responded to this need within the following technical response(s):

- A9143, HLW Tank Corrosion Control and Monitoring, TFA priority \#1.

\section{Site: INEEL}

Site Need ID: ID-2.1.06

Site Need Title: TRU, Cs and Sr Removal from High Activity Wastes

Need Summary: The removal of radionuclides may be accomplished via solvent extraction technology which has been under development by the DOE-EM programs for more than ten years. The TRUEX and SREX processes will be developed to provide working flowsheets for demonstration and implementation in centrifugal contactor equipment. This chemistry and flowsheet design needs to be developed, demonstrated, and verified with actual waste feed streams. Current baseline flowsheets for the TRUEX process utilize fluoride containing scrub reagents as well as phosphate containing strip reagents. Recent TFA vitrification formulation development efforts indicate that the phosphate results in excessive HLW glass volume (glass volume is limited by phosphate loading). Preliminary estimates also indicate that in the absence of phosphate in the strip solution, the fluoride resulting from the scrub reagent will then become the limiting factor in glass volume. Flowsheet development based on reduction or elimination of phosphate and fluoride in the TRUEX process is necessary to reduce HLW glass volumes to acceptable levels.

Cesium removal will be accomplished with an inorganic sorbent, ammonium molybdophosphate-polycrylonitrile (AMP-PAN), which has been demonstrated to have a high capacity and selectivity for cesium. Larger-scale column tests are needed to verify scaleup and design equations. An alternative and promising approach to using 3 discrete unit operations to remove $\mathrm{Cs}, \mathrm{Sr}$, and actinides is the universal solvent extraction (UNEX) process, which has been demonstrated to effectively remove radionuclides to Class A LLW levels. The UNEX process is especially amendable to treatment of the tank waste, because it requires much less cell space and capital costs to implement. Othe adjustments can be made 
to the UNEX process to remove only $\mathrm{Cs}$ and $\mathrm{Sr}$ and leave the raffinate as a TRU waste, if disposal to the Waste Isolation Pilot Plant (WIPP) is a preferred path.

Technical Disposition: The TFA responded to this need within the following technical response(s):

- A9501, INEEL Integrated Radionuclide Separations Process, TFA priority \#24.

Site Need ID: ID-2.1.15

Site Need Title: Neutralization of Newly Generated Liquid Wastes

Need Summary: Decontamination, decommissioning, analytical, and process operations at the Idaho Nuclear Technology and Engineering Center (INTEC) generate hazardous radioactive liquid waste. This newly-generated liquid waste (NGLW) has, traditionally, been combined with existing sodium-bearing wastes (SBW) stored in the tank farm, which is not fully compliant with modern environmental regulations requiring double containment of wastes. Based on the current operating assumptions, all of the SBW cannot be calcined by the end of 2012, as required by the Idaho Settlement Agreement. Aggressive reductions in the waste generation rates and/or segregation of NGLW will be required to meet the 2012 requirement. After evaporation, NGLW accounts for 100,000 to 150,000 gallons of wastewater added to the tanks each year. Much of the waste contains high sodium or potassium levels, which create solutions that must be diluted with cold chemicals to be. compatible with the calcination process; thus increasing the final waste volumes. If the NGLW were segregated from the existing SBW and neutralized to a non-corrosive $\mathrm{pH}(2.0<$ $\mathrm{pH}<12.5$ ), then it could be stored in an unused, spare tank until it was immobilized in grout or some other waste form. The existing tank vault around the spare stainless steel tank could qualify as compatible secondary containment if the waste is non-corrosive.

Varied concentrations of dissolved transition metal and aluminum salts (usually nitrate) pose challenges to prevent or redissolve precipitates during the neutralization process without causing undue equipment fouling, plugging, or erosion. The candidate neutralizing agents will be identified and solution stability determined during a study in FY99, but how and where the agents will be added needs to be determined. Process equipment, instrumentation, and materials of construction must be tested with a scaled mock-up so that recommendations can be made for full-scale process implementation. The physical location of process equipment and interfaces with existing facilities must be determined to facilitate segregating the NGLW from the existing wastewaters.

Technical Disposition: The TFA responded to this need within the following technical response(s):

- A9502, Neutralization of Newly Generated Liquid Wastes. The TFA did not develop a full technical response for this need as INEEL indicated the need would be withdrawn.

Site Need ID: ID-2.1.16

Site Need Title: Decontamination Facility/Analytical Facility Waste Reduction Need Summary: Processes operating at the INTEC generate hazardous radioactive liquid waste that is stored in the tank farm. Based on the current operating assumptions, all of this waste cannot be calcined by the end of 2012, as required by the Idaho Settlement Agreement. Aggressive reductions in waste generation rates will be required to meet the 2012 Settlement 
Agreement requirement. More efficient decontamination technologies and alternative operating techniques are currently being investigated as part of the EM-30 funded HLW Development Program (WBS \# 1.6.01.8.P0 and 1.6.01.8.T0, ADS 1008). However, implementation of the improvements required to achieve the reductions is needed to meet the goals of the agreement. Currently 100,000 to 150,000 gallons of additional waste (after evaporation) are added to the tank farm each year. In addition, much of this waste contains high sodium or potassium levels that create solutions that must be diluted with cold chemicals to allow calcination, thus increasing final waste volumes. The overall scope of this need is the reduction of waste (radioactive and mixed) from decontamination activities, the optimization of analytical processes and techniques, and the development/implementation of alternative waste stream treatments.

In addition, in FY 1998 INTEC and DOE-ID signed a 5-year Waste Minimization Incentive. This incentive will pay up to $\$ 5$ million to the INEEL M\&O contractor over the incentive period if an overall $43 \%$ waste reduction (about a 320,000 gallon reduction in waste generation) is met.

Technical Disposition: The TFA responded to this need within the following technical response(s):

- A9264, Improve Waste Analytical Methods, TFA priority \#37.

- A9508, Decon Process Waste Volume Reduction, TFA priority \#28.

Site Need ID: ID-2.1.17

Site Need Title: Develop New Filter Leach Process

Need Summary: The high-efficiency particulate air (HEPA) filter leach system at the INTEC generates hazardous radioactive liquid waste that is stored in the tank farm. Based on the current operating assumptions, all of the tank farm waste cannot be calcined by the end of 2012, as required by the Idaho Settlement Agreement. Aggressive reductions in waste generation rates will be required to meet the 2012 Settlement Agreement requirement. The current process, which leaches the used mixed waste HEPA filters with nitric acid to remove the RCRA components, is one of the larger waste streams still being sent to the tank farm.

In addition, in FY 1998, INTEC and DOE-ID signed a 5-year Waste Minimization Incentive. This incentive will pay up to $\$ 5$ million to the INTEC M\&O Contractor over the incentive period if an overall $43 \%$ waste reduction (about a 320,000 gallon reduction in waste generation) is met.

Technical Disposition: The TFA responded to this need within the following technical response(s):

- A9508, Decon Process Waste Volume Reduction, TFA priority \#28.

Site Need ID: ID-2.1.19

Site Need Title: EPA Methods Sample Collection and Analysis Verification/Development Need Summary: Approximately 1.4 million gallons of radioactive liquid waste is currently stored in 11 tanks at the INTEC. None of the tanks meet RCRA requirements for double containment, and several of the tanks (those with pillar and panel constructed vaults) do not meet seismic requirements. This is of great concern because the tanks are located over an aquifer. In response to a Notice of Noncompliance regarding this issue, the INEEL is bound 
by a Consent Order and a Settlement Agreement with the State of Idaho to cease use of these tanks by specific dates; 2009 for pillar and panel tanks and 2012 for all other tanks. The Consent Order was modified on August 18, 1998, thereby accelerating RCRA closure of the tanks. The Settlement Agreement also requires that all HLW at INTEC, including the solids in the Calcine Solids Storage Facility (CSSF), be treated to make it "road ready" for shipment to a repository for storage. This will involve retrieving the calcine and treating it at INTEC in some manner to ensure that all hazardous constituents are immobilized in a final waste form.

Both the liquid radioactive wastes in the tank farm and the calcine solids stored in the CSSF are classified as mixed radioactive-hazardous wastes. Existing and future processes to treat these wastes require the appropriate RCRA permits. When any of these wastes, or their derivatives, are treated in such a manner to produce an offgas stream, then offgas sampling will likely be required to verify compliance with RCRA risk assessment calculations. EPA sampling will especially be required when the wastes are treated by thermal processes, such as evaporation, denitration, and vitrification. These processes have the potential for emitting RCRA hazardous volatile and semi-volatile organics and volatile or particulate heavy metals. Some processes, such as waste incineration or calcination, may also generate RCRA organic compounds via de novo processes. All future thermal treatment processes may, in fact, come under the forthcoming Maximum Achievable Control Technology (MAXT) rule for incinerators.

In order to verify compliance with RCRA permits, EPA will require a trial burn, during which EPA sampling methods for volatile organics, semi-volatile organics, and volatile metals will be used to ensure compliance. Testing is needed to demonstrate that the EPA methods are valid for moist offgas that also has a high partial pressure of nitrogen oxides (NOx) and acid vapors. It may be necessary to adapt the EPA methods and laboratory analysis techniques for some streams. Testing should address sampling accuracy, precision, results reproducibility, and constituent detection limits.

Demonstration of the EPA Methods on INTEC pilot plant processes will ensure that the processes can be successfully permitted and operated. If modifications to the EPA sampling methods are required, than a timely ruling from EPA can be sought prior to start up of the processes.

Technical Disposition: The TFA responded to this need within the following technical response(s):

- A9206, EPA Methods Sample Collection and Analysis. The role of the Office of Science and Technology (OST) should be to monitor the evolution of sampling, analysis, and monitoring requirements for the other thermal processes. Discussions are to be continued with INEEL site personnel, Mixed Waste Focus Area, and CMST Crosscutting Program personnel as requirements become better defined. A collaborative workshop to identify a path forward should be convened if new requirements indicate a need for the development of new technologies over the current approach of adapting existing methodology. No FY01 funding of this technical approach is required beyond the normal technical assistance activities of OST and operational activities of INEEL. 
Site Need ID: ID-2.1.20

Site Need Title: Tank Annulus/Vault Inspection

Need Summary: Approximately 1.4 million gallons of radioactive liquid waste is currently stored in 11 tanks at the INTEC. None of the tanks meet RCRA requirements for double containment and several of the tanks (those with pillar and panel constructed vaults) do not meet seismic requirements. This is of great concern because the tanks are located over an aquifer. In response to a Notice of Noncompliance regarding this issue, the INEEL is bound by a Consent Order and a Settlement Agreement with the State of Idaho to cease use of these tanks by specific dates; 2009 for pillar and panel tanks and 2012 for all other tanks. The Consent Order was modified on August 18, 1998, thereby accelerating RCRA closure of the tanks. A closure plan must be submitted to the Idaho Department of Environmental Quality by December 31, 2000. In addition, 2 of the tanks, WM-182 and WM-183, are scheduled for early closure by 2003 . Tank closure acceptance criteria need to be developed to meet the RCRA Landfill Closure Standards and State approval in support of the closure plans. This requires not only development of criteria, but also development of the process needed to ascertain compliance with those criteria.

The Light Duty Utility Arm (LDUA) was deployed in tanks at the INEEL beginning in FY 1999. However, LDUA is too big to access the tank annulus and vaults, so they have not been, and cannot currently be, adequately inspected. Inspection of the vaults is required to ascertain tank integrity from the outside of the tank, inspect the vault sumps, sample the sumps, and inspect the sand pad under at least two of the tanks. The two tanks in question experienced releases to the vault, wetting the sand pad under the tanks. The effect of subsequent water flushes on the wetted sand pad, sumps and vaults, has not been quantified. Quantification of the risk is vital to RCRA and CERCLA closure of the vaults.

Technical Disposition: The TFA responded to this need within the following technical response(s):

- A9175, Tank Integrity Inspection Techniques, TFA priority \#11.

- AA1S1, Pre-Closure Interim Tank Maintenance, TFA strategic task.

Site Need ID: ID-2.1.23

Site Need Title: Low-Activity Wasteform Qualification

Need Summary: In-depth information, program costs, and lessons learned are needed from operating sites concerning how to perform and complete wasteform qualification for grouted mixed LLW. This includes qualification of the grouting process as well as the final wasteform.

Technical Disposition: The TFA responded to this need within the following technical response(s):

- A9719, Conditioning and Immobilization of Low-Activity Waste, TFA priority \#14.

Site Need ID: ID-2.1.24

Site Need Title: Integration/Optimization of High Activity Waste/Low Activity Waste Process Flowsheet

Need Summary: Many alternatives and options are being considered for the treatment and qualification of radioactive wastes located at INTEC for permanent disposal. Adequate 
evaluation of these options requires that each one have a process flow diagram and associated mass and energy balance. These are called flowsheets. The flowsheets provide the technical basis for performing cost estimates, safety evaluations, and estimates of impact to the environment. Later, they provide the technical basis for permit applications. Presently, the flowsheet calculations are performed manually, or with the assistance of several different software applications. These calculations must also be performed in the same manner again as new data is obtained which clarify assumptions that have been made. They are normally done for just one unit operation and do not link all of the required operations into a process flowsheet.An integrated simulation tool(s) is needed to perform these calculations automatically, with minimal effort on the part of the engineer(s) who are tasked with doing this work. This tool(s) would consist of both software and unit operation mathematical models. This provides for more accurate and timely data required for further evaluations. Technical Disposition: The TFA responded to this need within the following technical response(s):

- A9709, Waste Treatment Process Flowsheet Model, TFA priority \#7.

Site Need ID: ID-2.1.25

Site Need Title: Ion-Exchange System for Water Runoff

Need Summary: Processes operating at INTEC generate hazardous radioactive liquid waste that is stored in the tank farm. Based on the current operating assumptions, all of this waste cannot be calcined by the end of 2012, as required by the Idaho Settlement Agreement. Aggressive reductions in waste generation rates will be required to meet the 2012 Settlement Agreement requirement. More efficient decontamination technologies and alternative operating techniques are currently being investigated as part of the EM-30 funded HLW Development Program (WBS \# 1.6.01.8.P0 and 1.6.01.8.T0, ADS 1008). However, implementation of the improvements required to achieve the reductions is needed to meet the goals. Currently 100,000 to 150,000 gallons of additional waste (after evaporation) are added to the tank farm each year. In addition, much of this waste contains high sodium or potassium levels that create solutions that must be diluted with cold chemicals to allow calcination, thus increasing final waste volumes. The overall scope of this need is the reduction of waste (radioactive and mixed) from decontamination activities, the optimization of processes, and the development/implementation of alternative waste stream treatments.

In addition, in FY 1998 LMITCO and DOE-ID signed a 5-year Waste Minimization Incentive. This incentive will pay up to $\$ 5$ million to LMITCO over the incentive period if an overall $43 \%$ waste reduction (about a 320,000 gallon reduction in waste generation) is met. Technical Disposition: The TFA responded to this need within the following technical response(s):

- A9510, Ion-Exchange System for Water Runoff. The TFA did not develop a full technical response for this need as INEEL indicated the need would be withdrawn.

Site Need ID: ID-2.1.26

Site Need Title: Direct Tank Sampler for Tank Solution Characterization Need Summary: A method for sampling waste from waste tanks at the INTEC tank farm Facility is needed to fully characterize the waste stored in the tanks, while meeting RCRA 
requirements for representative sampling and minimization of sample degradation during sampling. Currently, the waste is transferred from the tank farm tank to a tank at the New Waste Calcining Facility via a steam jet or airlift, sparged, and then sampled via a sampler which uses an air jet to pull liquid through a sample bottle. The existing remote sampler system and sample transport system is also designed for small $15 \mathrm{ml}$ to $50 \mathrm{ml}$ sample bottles, which do not fill to zero head space and must be recombined in the laboratory remote cell for the larger volumes required for the EPA analyses. There is concern with the representiveness of the sample and loss of volatile organics during jet/airlift transfer, sparging, and sampling.

The State of Idaho recently requested that permitting of facilities at INTEC be accelerated, and RCRA-compliant waste characterization is needed prior to permitting of the facilities. Technical Disposition: The TFA responded to this need within the following technical response(s):

- A9246, Waste Sampling and At-Tank Analysis, TFA priority \#21.

Site Need ID: D-2.1.27

Site Need Title: Blowback Metal Filters for Solids (Calcine) Retrieval

Need Summary: Highly radioactive waste material in the form of calcine is stored in bins in seven CSSFs. The Settlement Agreement requires a plan that provides for treatment of all calcined waste to produce a waste form which is suitable for transport to a permanent repository. The material will be transferred to a new processing facility by a dilute phase vacuum pneumatic transport system to meet this requirement. After the solids are separated from the transport air, the air will be HEPA filtered. Used HEPA filters would be a mixed waste. A filter leaching process may be used to remove the hazardous contaminants from the filters, converting them to a LLW. A solids separation system, which minimizes the expense of disposing of used HEPA filters, is needed.

Technical Disposition: The TFA responded to this need within the following technical response(s):

- A9171, Alternative Air Filtration Technology, TFA priority \#15.

Site Need ID: ID-2.1.28

Site Need Title: Cs and Sr Removal from Newly Generated Liquid Waste Need Summary: Sorption chemistry and large scale column designs need to be developed, demonstrated, and verified with actual waste feed streams at the INEEL. Cesium removal will be accomplished with an inorganic sorbent, primarily CST. Stronium removal must be developed either at the acid concentration of the evaporator bottoms or following partial neutralization. Sorption isotherms and column breakthrough tests must be performed to determine sorbent capacity and develop column design parameters.

Technical Disposition: The TFA responded to this need within the following technical response(s):

- A9570, Salt Disposition, TFA priority \#6.

- A9719, Conditioning and Immobilization of Low-Activity Waste, TFA priority \#14.

Site Need ID: ID-2.1.29

Site Need Title: Evaluate Chloride Corrosion Potential (LET\&D/PEWE/Future Processes) 
Need Summary: Waste generated at INTEC has historically been kept acidic (primarily nitric acid) to facilitate further treatment such as evaporation or calcination. The high nitrate concentration and acidic conditions provide the ability to dilute high chloride waste with low chloride wastes for storage and processing without significant corrosion problems. All waste going to the INTEC liquid waste systems are being minimized such that there is less low chloride waste, while processing of the existing waste in the HLW evaporator and New Waste calciner still generate waste with high chloride concentrations. In the past, chloride concentrations for wastes going to the Process Evaporative Waste Evaporator have been less than $50 \mathrm{mg} / \mathrm{L}$ chloride. With future processing, waste concentrations are expected to be upwards of $250 \mathrm{mg} / \mathrm{L}$ chloride. Significant corrosion has already been experienced in the offgas system for the Liquid Effluent Treatment and Disposal (LET\&D) acid fractionator which processes the overheads from the PEWE. Future waste processing operations must be modeled, and the effect of the higher chloride concentrations on equipment service life must be evaluated.

Technical Disposition: The TFA responded to this need within the following technical response(s):

- A9514, Removal of Chloride from Waste Solutions, TFA priority \#43.

Site Need ID: ID-2.1.30

Site Need Title: Remove/Treat Chlorides (LET\&D/PEWE/Future Processes)

Need Summary: Waste generated at INTEC has historically been kept acidic (primarily nitric acid) to facilitate further treatment such as evaporation or calcination. The high nitrate concentration and acidic conditions provide the ability to dilute high chloride waste with low chloride wastes for storage and processing without significant corrosion problems. However, all waste going to the INTEC liquid waste systems are being minimized such that there is less low chloride waste, while processing of the existing waste in the HLW evaporator and New Waste calciner still generate waste with high chloride concentrations. Current tank farm wastes have up to $1500 \mathrm{mg} / \mathrm{L}$ chloride. In the future, wastes are projected to have up to 5000 $\mathrm{mg} / \mathrm{L}$ chloride. This will cause corrosion problems during storage. In the past, chloride concentrations for wastes going to the Process Evaporative Waste Evaporator (PEWE) have been less than $50 \mathrm{mg} / \mathrm{L}$ chloride because dilute chloride wastes were available. With future processing, waste concentrations for PEWE are expected to be upwards of $250 \mathrm{mg} / \mathrm{L}$ chloride and due to waste minimization efforts, less low chloride wastes will be available to dilute this waste stream. Significant corrosion has already been experienced in the off-gas system for the Liquid Effluent Treatment and Disposal (LET\&D) acid fractionator, which processes the overheads from the PEWE. Some form of chloride removal or treatment will be necessary to process future wastes. A literature evaluation must be completed in FY00 and a laboratory evaluation must be completed in FY01 to support the INEEL HLW Program schedule. Technical Disposition: The TFA responded to this need within the following technical response(s):

- A9514, Removal of Chloride from Waste Solutions, TFA priority \#43.

Site Need ID: ID-2.1.31

Site Need Title: Characterization of Entrainable Solids in Tank Waste

Need Summary: Little or no characterization of in-tank, entrainable solids at INTEC has been performed. Analysis of these solids must be performed in order to select the proper 
solid-liquid separation technology. Various factors such as particle size and concentration greatly affect the separation process, and must be considered in the design stage. A solidliquid separation technology cannot be chosen or implemented until these factors are determined.

Technical Disposition: The TFA responded to this need within the following technical response(s):

- A9216, Characterization of Entrainable Solids. The TFA did not develop a full technical response for this need based on TFA discussions with INEEL from last year and continuing this year. The TFA determined that there is little or no R\&D component associated with the need. INEEL indicated that the tank waste undissolved solids would be dissolved and routine hot-cell methods in the RAL would be used for analysis. The TFA recommends that INEEL reassess this need in the next site needs submission cycle.

Site Need ID: ID-2.1.35

Site Need Title: Direct Immobilization of INTEC Sodium-Bearing and Newly Generated Liquid Wastes

Need Summary: A method to directly immobilize and stabilize SBW and NGLW are needed. SBW is extremely acidic and high in nitrates, sodium, and aluminum and was produced by highly evaporating NGLW. NGLW is produced by decontamination operations and process equipment wastes. "Direct" means the liquid waste would be added to the solidifying agents, such as grout, with limited processing (evaporation, acid neutralization, and specific radionuclide separation). The waste product must meet applicable waste acceptance criteria for TRU waste or LLW. Two methods of direct grouting SBW using portland cement, blast furnace slag, and fly ash have been tested at 40 weight percent waste. These methods were tested on NGLW during FY 1999. INEEL has to find a method to improve waste loading and reduce total waste volume.

Technical Disposition: The TFA responded to this need within the following technical response(s):

- A9719, Conditioning and Immobilization of Low-Activity Waste, TFA priority \#14.

Site Need ID: ID-2.1.36

Site Need Title: Mercury Removal from Liquid Wastes

Need Summary: The liquid radioactive waste being stored at INTEC contains mercury (Hg).

Processing this waste by vitrification, calcination, or by proposed separations processes

involving denitration of HAW and LAW will volatilize greater than $90 \%$ of the $\mathrm{Hg}$.

Measurements made during past calcination campaigns have indicated that 1) $\mathrm{Hg}$ accumulates in offgas scrub solutions, and 2) mercury emissions from calcination will exceed future limits expected to be imposed by the new Maximum Achievable Control Technology (MAXT) rules. Technology is required to remove $\mathrm{Hg}$ from offgas scrub solutions in order to reduce $\mathrm{Hg}$ emissions, decrease the $\mathrm{Hg}$ load on downstream mercury sorbents, and reduce $\mathrm{Hg}$ buildup in stored scrub solutions. A basic understanding of the behavior of $\mathrm{Hg}$ in nitric acid solutions containing chloride is required in order to develop a $\mathrm{Hg}$ removal method. 
Technical Disposition: The TFA responded to this need within the following technical response(s):

- A9518, Mercury Removal from Waste Solutions, TFA priority \#46.

Site Need ID: ID-2.1.38

Site Need Title: Conditioning of Low Activity Waste for Treatment

Need Summary: HLW calcine and sodium-bearing wastewater at the INTEC require conditioning and treatment prior to storage/disposal in an approved repository. To minimize the volume of remotely handled HLW that must be discarded, the waste steams will be separated into HAW and LAW fractions, using one or more chemical processes. Calcine will be retrieved from storage bins, dissolved in nitric acid, and processed to remove most of the non-radioactive constituents, which will constitute the LAW fraction. Current expectations are that the LAW fraction will be immobilized on-site in a portland-cement based grout. However, an EIS alternative is to ship the conditioned LAW off-site for immobilization. Conditioning will be required to curb the ultimate volume of LAW grout and to ensure that the grout will properly cure and meet performance criteria. Composition of the LAW fraction would vary depending on the feed solutions that are processed through the separations plant. Technical Disposition: The TFA responded to this need within the following technical response(s):

- A9719, Conditioning and Immobilization of Low-Activity Waste, TFA priority $\# 14$.

Site Need ID: ID-2.1.39

Site Need Title: Acceptance Criteria for LAW Disposal in Underground Storage Tanks Need Summary: Waste acceptance criteria must be developed and approved to use the underground storage tanks as low-level Class A waste disposal facilities. Any operating experience or lessons learned from other sites on this subject is needed.

Assumption: It is assumed that the tanks would need to be licensed as a LLW landfill by the US Nuclear Regulatory Commission.

Technical Disposition: The TFA responded to this need within the following technical response(s):

- A9924, Tank Closure Criteria/Decision Support, TFA priority \#30.

Site Need ID: ID-2.1.40

Site Need Title: Low Activity Waste Grout Sorbent Addition to Reduce Leachability Need Summary: Research information is needed concerning the addition of chemical sorbents to grouted waste to reduce the leachability of radionuclides and RCRA metals from the waste.

Technical Disposition: The TFA responded to this need within the following technical response(s):

- A9719, Conditioning and Immobilization of Low-Activity Waste, TFA priority \#14.

Site Need ID: $\mathbb{D}-2.1 .41$

Site Need Title: HLW Process Offgas Treatment 
Need Summary: Approximately 1.4 million gallons of radioactive liquid waste is currently stored in 11 tanks at the INTEC. None of the tanks meet RCRA requirements for double containment and several of the tanks (those with pillar and panel constructed vaults) do not meet seismic requirements. This is of great concern because the tanks are located over an aquifer. In response to a Notice of Noncompliance regarding this issue, the INEEL is bound by a Consent Order and a Settlement Agreement with the State of Idaho to cease use of these tanks by specific dates; 2009 for pillar and panel tanks and 2012 for all other tanks. The Consent Order was modified on August 18, 1998, thereby accelerating RCRA closure of the tanks.

The Settlement Agreement also requires that all HLW at INTEC, including the solids in the Calcine Solids Storage Facility (CSSF) be treated to make it "road ready" for shipment to a repository for storage. This will involve retrieving the calcine and treating it at INTEC in some manner to ensure that all hazardous constituents are immobilized in a final waste form.

Offgas treatment technology development is needed to support HLW treatment technology development and feasibility design studies. The HLW wastes and their derivatives are considered RCRA wastes because they contain hazardous organic compounds and heavy metals. Future HLW thermal treatment processes, including the exiting NWCF (which may continue to be used to calcine existing tank farm liquid wastes), have the potential of emitting products of incomplete combustion (PICs), RCRA hazardous organic compounds (aldehydes, ketones, poly-aromatic compounds, halogenated and nitrated compounds, dioxins/furans, etc.), heavy metals, and criteria pollutants ( $\mathrm{CO}, \mathrm{NOx}$, and fine particulate). Offgas treatment processes need to be identified, tested, and designed to control emissions of any or all of these pollutants. Emissions limits must be compliant with the Clean Air Act (CAA), the RCRA, and the forthcoming MAXT rule. It may be necessary to control NOx in the offgas in order to remove $\mathrm{Hg}$, since high levels of $\mathrm{NOx}$ will likely interfere with the candidate $\mathrm{Hg}$ removal technologies.

This needs statement addresses the development of control technologies for PICs and NOx, whereas, control technologies for $\mathrm{Hg}$ emissions are addresses in a separate needs statement. Abatement technologies for particulate and acid vapors are considered mature; therefore, a survey of commercially available control systems is needed to identify the best of existing technologies to include in process feasibility studies. Consideration must be given to the fact that these are radiological processes.

Technical Disposition: The TFA responded to this need within the following technical response(s):

- A9722, HLW Process Offgas Treatment. The TFA did not develop a full technical response for this need as it is currently being addresses by the Mixed Waste Focus Area by testing being performed at the MSE facilities in Butte, Montana.

Site Need ID: ID-2.1.42

Site Need Title: Acceptance Criteria for Tank Closure

Need Summary: Approximately 1.4 million gallons of radioactive liquid waste is currently stored in 11 tanks at the INTEC. None of the tanks meet RCRA requirements for double 
containment and several of the tanks (those with pillar and panel constructed vaults) do not meet seismic requirements. This is of great concern because the tanks are located over an aquifer. In response to a Notice of Noncompliance regarding this issue, the INEEL is bound by a Consent Order and a Settlement Agreement with the State of Idaho to cease use of these tanks by specific dates; 2009 for pillar and panel tanks and 2012 for all other tanks. The Consent Order was modified on August 18, 1998, thereby accelerating RCRA closure of the tanks. A closure plan must be submitted to the Idaho Department of Environmental Quality (DEQ) by December 31, 2000. In addition, two of the tanks, WM-182 and WM-183, are scheduled for early closure by 2004 . Tank closure acceptance criteria need to be developed to meet the RCRA Standard Requirements and State approval in support of the closure plans. This requires not only development of criteria, but also development of the process needed to ascertain compliance with those criteria.

Technical Disposition: The TFA responded to this need within the following technical response(s):

- A9924, Tank Closure Criteria/Decision Support, TFA priority \#30.

Site Need ID: ID-2.1.43

Site Need Title: Certify LDUA Sampler as EPA-Approved Method of Sampling Tank Heel Liquids

Need Summary: Approximately 1.4 million gallons of radioactive liquid waste is stored in 11 tanks at the INTEC. None of the tanks meet RCRA requirements for double containment and several of the tanks (those with pillar and panel constructed vaults) do not meet seismic requirements. In response to a Notice of Noncompliance, a Consent Order and Settlement Agreement with the State of Idaho, the INEEL is bound to close the tanks. The Consent Order was modified on August 18, 1998, accelerating the tank closure schedule. The INEEL is required to cease use of the five pillar and panel tanks by June 2003, and cease use of the others by 2012 . The first tank closure plan must be submitted by December 31,2000 , and the integrity of one of the tanks must be verified so it can be used as an emergency spare.

The Consent Order dictates that the INEEL shutdown the calciner process currently used to solidify tank farm wastes, and place it in standby mode pending a Record of Decision (ROD) on the site's EIS. However, evaporative and fractionation processes will continue to operate. A delisting petition will be submitted for the liquid waste that these processes treat, which will require extensive knowledge of the liquid waste constituents.

There have been some minor releases from the tank farm during its history of operation. CERCLA closure requires that the releases be quantified to define a source term and relative risk. Extensive knowledge of the liquid and solid waste constituents is required to define the source term.

Sampling the tank farm waste is required to support tank closure, delisting, an incidental waste determination, and CERCLA source term definition. A new method of directly sampling the tank farm heels and solution with a robotic arm was demonstrated. However, not all of the analytes required for the listed initiatives have methods developed and implemented at the INTEC analytical laboratories. High radiation fields necessitate the use of remote methods employed in hot cells to analyze the liquid and solids samples, making it 
difficult or impossible to use standard equipment and procedures to perform analyses strictly in accordance with SW-846 protocol.

Deviations from approved protocol, and total lack of analysis for some analytes, significantly impact the site's ability to compile a comprehensive data package. Development and demonstration of EPA-approved methods significantly enhance INTEC's ability to perform RCRA and CERCLA closure, analyze all DOE Order 435.1 radionuclides of concern, and provide required data to develop new process flow sheets mandated by the Settlement Agreement and the Notice of Noncompliance (NON) consent order.

Site wide, the need to sample and analyze these same analytes is wide spread because most are termed constituents of concern in DOE Order 435.1. Since most HLW must be analyzed remotely, has significant solids associated with it, and must be analyzed in accordance with DOE Order 435.1, improved sample analysis methods will greatly benefit the complex. Technical Disposition: The TFA responded to this need within the following technical response(s):

- A9246, Waste Sampling and At-Tank Analysis, TFA priority \#21.

Site Need ID: ID-2.1.44

Site Need Title: Certify LDUA Sampler as EPA-Approved Method of Sampling Tank Heel Solids

Need Summary: Approximately 1.4 million gallons of radioactive liquid waste is stored in 11 tanks at the INTEC. None of the tanks meet RCRA requirements for double containment and several of the tanks (those with pillar and panel constructed vaults) do not meet seismic requirements. In response to a Notice of Noncompliance, a Consent Order and Settlement Agreement with the State of Idaho, the INEEL is bound to close the tanks. The Consent Order was modified on August 18, 1998, accelerating the tank closure schedule. The INEEL is required to cease use of the five pillar and panel tanks by June 2003, and cease use of the others by 2012 . The first tank closure plan must be submitted by December 31,2000 , and the integrity of one of the tanks must be verified so it can be used as an emergency spare.

The Consent Order dictates that the INEEL shutdown the calciner process currently used to solidify tank farm wastes, and place it in standby mode pending the EIS ROD. However, evaporative and fractionation processes will continue to operate. A delisting petition will be submitted for the liquid waste that these processes treat, which will require extensive knowledge of the liquid waste constituents.

There have been some minor releases from the tank farm during its history of operation. CERCLA closure requires that the releases be quantified to define a source term and relative risk. Extensive knowledge of the solid waste constituents is required to define the source term.

Sampling the tank farm waste is required to support tank closure, delisting, an incidental waste determination, and CERCLA source term definition. Currently, the tank farm waste is sampled after transfer to an adjacent facility. However, the solution must be transferred by steam jet and air lifted to the sampler. The jet does not sample the heel solids as they collect 
in the tanks below the suction leg. Therefore, there is not even operational knowledge of the solids chemical and physical properties. A new method of directly sampling the tank farm heels and solution with a robotic arm was demonstrated. However, the sampler employed by the arm experienced some plugging problems while sampling WM-182, a tank expected to hold relatively few solids.

More solids are expected in tanks that will be sampled later. Additionally, the sampler was designed to minimize solids collection because at the time of design, ALARA concerns were deemed more important than solids collection. Hence, the sampler will not always draw adequate solids to perform all required analyses, and the solids collected are not necessarily representative of the tank heel.

Modification of the sampler will provide a sample truly representative of the tank heel solids and allow INTEC to employ the robotic arm in sampling wastes to support tank closure, WIR determination, delisting and CERCLA source term definition. Demonstration of the sampler's ability to draw a representative solids sample will gain acceptance of the analytical . results from the EPA, and provide reliable information to technical development groups, CERCLA and DOE.

Technical Disposition: The TFA responded to this need within the following technical response(s):

- A9246, Waste Sampling and At-Tank Analysis, TFA priority \#21.

Site Need ID: ID-2.1.45

Site Need Title: Acceptance Criteria for Grouting Tank Heels

Need Summary: Upon tank closure, there will be some process residual waste, called a heel, left in the bottom of each tank. The closure acceptance criteria for the tank heels is needed to design tank closure and determine the method to immobilize and stabilize the heels. SRS and ORR sites have closed a few tanks. Any licensing and operating experience or lessons learned from these sites is needed.

Technical Disposition: The TFA responded to this need within the following technical response(s):

- A9924, Tank Closure Criteria/Decision Support, TFA priority \#30.

Site Need ID: ID-2.1.46

Site Need Title: Management of Tank Heel Liquids

Need Summary: Approximately 1.4 million gallons of radioactive liquid waste is currently stored in 11 tanks at the INTEC. None of the tanks meet RCRA requirements for double containment and several of the tanks (those with pillar and panel constructed vaults) do not meet seismic requirements. This is of great concern because the tanks are located over an aquifer. In response to a Notice of Noncompliance regarding this issue, the INEEL is bound by a Consent Order and a Settlement Agreement with the State of Idaho to cease use of these tanks by specific dates; 2009 for pillar and panel tanks and 2012 for all other tanks. The Consent Order was modified on August 18, 1998, thereby accelerating RCRA closure of the tanks. A closure plan must be submitted to the Idaho Department of Environmental Quality (DEQ) by December 31, 2000. In addition, two of the tanks, WM-182 and WM-183, are scheduled for early closure by 2004 . 
Currently, the tanks can only be emptied to the heel level, due to the level of the steam jets used to empty the tanks. Therefore, several thousand gallons of waste will still remain in the tanks when they are no longer being used. The site plans to close these tanks by grouting the heels in place. This poses some technical and regulatory challenges. The liquid heels are acidic and may not be conducive to direct grouting due to the chemistry and lack of mixing capabilities. In addition, acceptance criteria and waste form qualification for the solidified heels may prove difficult to meet for insitu grouting. In order to form a grouted waste and meet RCRA Closure Standards and State negotiated acceptance criteria, the liquid heels may have to be diluted, neutralized, reduced in volume, or totally removed. There is currently no mechanism to accomplish either neutralization or liquid heel removal, nor has it been proven that the liquid heel can be grouted in place. Therefore, development work is needed to first determine what must be done to manage these liquids to meet tank closure criteria (dilution, neutralization, reduced in volume, removed, etc.) and then how that can physically be accomplished.

Technical Disposition: The TFA responded to this need within the following technical response(s):

- A9923, Enhanced Grout Formulations for Tank Closure, TFA priority \#10.

Site Need ID: ID-2.1.47

Site Need Title: Management of Tank Heel Solids

Need Summary: Approximately 1.4 million gallons of radioactive liquid waste is currently stored in 11 tanks at the INTEC. Currently, the tanks can only be emptied to the heel level, due to the level of the steam jets used to empty the tanks. Therefore, several thousand gallons of waste will still remain in the tanks when they are no longer being used. It is planned to close these tanks by grouting the heels in place. This poses some technical and regulatory challenges. The liquid heels are acidic and may not be conducive to direct grouting due to the chemistry and lack of mixing capabilities. In addition, acceptance criteria and waste form qualification for the solidified heels, and may prove difficult to meet for insitu grouting.

In order to form a grouted waste and meet RCRA Closure Standards and State negotiated acceptance criteria, the liquid heels may have to be diluted, neutralized, reduced in volume, or totally removed. There is currently no mechanism to accomplish either neutralization or liquid heel removal, nor has it been proven that the liquid heel can be grouted in place. Therefore, development work is needed to first determine what must be done to manage these liquids to meet tank closure criteria (dilution, neutralization, reduced in volume, removed, etc.) and then how that can physically be accomplished.

Technical Disposition: The TFA responded to this need within the following technical response(s):

- A9923, Enhanced Grout Formulations for Tank Closure, TFA priority \#10.

Site Need ID: ID-2.1.48

Site Need Title: Wasteform Qualification for Low-Activity Waste in Underground Storage

Tanks

Need Summary: In-depth grout development work will be required to determine

formulation and operational constraints which will provide acceptable curing conditions and simultaneously assure optimized final grout performance requirements (leachability, strength, 
etc.). In-depth information, program costs, and lessons learned are needed from operating sites concerning how to perform and complete wasteform qualification for grouted mixed LLW. This includes qualification of the grouting process as well as the final wasteform. Completion of HLW Technology Needs ID \# 2.1.39 (Acceptance Criteria for LAW Disposal in Tanks) will be required prior to full completion of this need.

Technical Disposition: The TFA responded to this need within the following technical response(s):

- A9924, Tank Closure Criteria/Decision Support, TFA priority \#30.

Site Need ID: ID-2.1.49

Site Need Title: Acceptance Criteria for High Activity Waste/Low Activity Waste Need Summary: High level radioactive waste (HLW) being stored at INTEC is not in a form suitable for repository storage. Vitrification, the Best Demonstrated Available Technology for converting HLW to a form suitable for storage in a federal geologic repository, has been reduced to practice at SRS's Defense Waste Processing Facility and at the WVDP. The DOE-RW Waste Acceptance System Requirements Document (WASRD) describes the system level requirements for emplacement of immobilized HLW in a federally licensed repository. The DOE-RM estblished Waste Acceptance Product Specifications (WAPS) defining technical and documentation requirements for vitrified waste forms to satisfy the higher level WASRD. Likewise, 10 CFR 61 establishes all requirements for the suitability of a grouted low activity waste forms such as INEEL/INTEC LAW to be disposed in shallow land burial. These precedents and requirements provide the drivers to convert INTEC HAW to a borosilicate glass and LAW to a grout suitable for storage in a federal geologic repository. Thus the needs of this program consist of the following items:

1. Evaluate the application of WASRD/WAPS and 10 CFR 61 requirements at other DOEcomplex sites for the purpose of adapting past experience to INEEL/INTEC needs

2. Establishment of an administrative system to collect information and data that proves the suitability vitrified and grouted products for respective disposal.

Technical Disposition: The TFA responded to this need within the following technical response(s):

- A9730, Acceptance Criteria for High Activity/Low Activity Waste. It does not appear that technology development is required to meet this need. Therefore, an approach to meet this need is for Idaho to meet with SRS and WVDP to understand how these sites developed methods and administrative systems for meeting the requirements necessary to demonstrate compliance prior to disposal of HLW and LAW. The results of current efforts at Hanford on disposal of their LAW in an on-site shallow land burial site would also be useful. This involves the iterative process between the Performance Assessment and the development of waste acceptance criteria. The TFA can provide technical assistance to ensure INEEL has the appropriate contacts at SRS, WVDP, and Hanford.

Site Need ID: D-2.1.50

Site Need Title: Solids Waste (Calcine) Retrieval

Need Summary: Highly radioactive waste material is being stored in bins in seven CSSF. The material was in the form of granular solids and fines when it was sent to storage. Some 
of the material may have formed a relatively weak crust or cake in storage. Systems are needed to retrieve the calcined solids out of storage bins and transfer them to a processing facility, so that they can be processed into an even more stable waste form.

Technical Disposition: The TFA responded to this need within the following technical response(s):

- A9331, Dry Solid Waste Retrieval, TFA priority \#31.

Site Need ID: ID-2.1.51

Site Need Title: Develop Calcine Dissolution Kinetics for Solid/Liquid Equilibria

Need Summary: Nitric acid dissolution of the calcine is one of the key head end operations in the separations option. This process requires investigation of the various rates occurring during dissolution, the extent of the dissolution, and the final solids/liquid equilibria associated with the dissolution process. Ideally, to scale-up and design calcine dissolution equipment, and dissolution reaction rate expression is required for integration in an appropriate reactor performance equation. This dissolution rate expression will model heterogeneous reaction and accommodate the possibilities for the rate controlling phenomena. This will be accomplished by integrating the following parameters: 1) the surface reaction rate constant, including temperature dependence; 2) the external film mass transfer coefficient, including dependence on agitation power input, and;3) the internal effective diffusion coefficient. These parameters are required for scale-up and design of a calcine dissolution reactor. In addition, this model will predict the extent of the dissolution and the dissolver product solids/liquid equilibria. Calcine dissolution work may provide useful information for on going operations in which calcine dissolution is required for other processes or equipment.

Technical Disposition: The TFA responded to this need within the following technical response(s):

- A9532, Calcine Dissolution Solubility and Kinetics, TFA priority \#48.

Site Need ID: ID-2.1.52

Site Need Title: Characterization of Solids from Calcine Dissolution

Need Summary: Available data indicates 90 to $98 \mathrm{wt} \%$ of the calcine at the INTEC can be dissolved in several hours using nitric acid (necessary for compatibility with the downstream separation processes), elevated temperature, and continuous mixing. The residual or undissolved solids (UDS) from the dissolution process must be segregated from the liquid stream input to the downstream separation process since they cause problems in operational aspects of the separation process and can provide a source of significant contamination in the LLW fraction from separations. The data obtained to date indicates the UDS from the dissolution process will be intensely radioactive, thus requiring disposal with the HLW fraction and emphasizing the need for efficient removal from the liquid dissolver product. In order to efficiently remove or filter the solids from the liquid stream, physical characteristics of the UDS, such as particle size distribution, must be determined. Physical characterization must be established prior to selecting a solids removal system. Due to the intense radioactivity of the solids, characterization must be performed in a remote environment. Finally, chemical characterization of the UDS is required to establish compatibility with the HLW final waste form. 
Technical Disposition: The TFA responded to this need within the following technical response(s):

- A9532, Calcine Dissolution Solubility and Kinetics, TFA priority \#48.

Site Need ID: ID-2.1.56

Site Need Title: Mercury Treatment for Aluminum Calcine

Need Summary: The removal of $\mathrm{Hg}$ may be accomplished via solvent extraction technology or ion exchange. The sorption chemistry and flowsheet design needs to be developed and demonstrated, as well as verified with actual waste feed streams. Hg distribution in TRUEX and SREX solvent extraction flowsheets will be verified. Removal of $\mathrm{Hg}$ from solvent wash streams (sodium carbonate) is also required.

Technical Disposition: The TFA responded to this need within the following technical response(s):

- A9501, INEEL Integrated Radionuclide Separations Process, TFA priority \#24.

- A9518, Mercury Removal from Waste Solutions, TFA priority \#46.

Site Need ID: ID-2.1.57

Site Need Title: Conditioning of HAW for Treatment

Need Summary: Processing INEEL HLW calcine and tank farm liquids by separations processes results in HAW streams that will undergo further treatment into one or more final waste forms. Various alternative separations processes are currently envisioned. One treatment system, referred to as the "TRU Separations Option," results in a HAW waste stream that contains the actinide strip solution from the TRUEX process and undissolved solids separated from the feed. These wastes are to be concentrated by evaporation and, if feasible, dried to a granular solid. Data is needed to determine the extent and method of evaporation and drying of this waste. If found infeasible to evaporate to dryness, data is needed to determine a method of stabilizing the waste into a solid form. In another treatment system referred to as "Full Separations," the TRUEX strip solution and the SREX strip solution are evaporated prior to being fed to a glass melter. Data is needed to determine the extent of evaporation achievable for these wastes, the point at which precipitates form, and the nature of the precipitated solids.

Technical Disposition: The TFA responded to this need within the following technical response(s):

- A9768, Specify and Enhance Design of HLW Glass Melters, TFA priority \#3.

- AA7S2, New Melter Technology, TFA strategic task.

Site Need ID: ID-2.1.58

Site Need Title: HAW Immobilization

Need Summary: Facilities planned for vitrification of high level wastes at the INTEC/INEEL will have to satisfy WAPS requirements, and it is the responsibility of the producer to develop the process that will produce a qualified product. The existing vitrification formulation data base must be expanded to successfully formulate, process, and qualify INEEL HLW. Operating experience at DWPF and WVDP reveals that propertycomposition relationships such as liquidus temperature and phase separation limit the extent of waste loading in glass. The HLW technology develoment program therefore has the purpose of: 
1. Developing the selection criteria needed to design and operate HLW vitrification processes that are capable of immobilizing and qualifying these wastes to "road ready" for repository storage by year 2035 as specified in the Batt Settlement Agreement between the State of Idaho, the USDOE and the US Navy,

2. Leveraging existing data and experience from other DOE sites to aid in the development of the INTEC/INEEL vitrification process requirements.

3. Acquiring data and information that will expand existing databases to provide information that will improve the models that control HLW vitrification processes. These expanded and updated models/databases can be leveraged to improve existing operations of vitrification processes as well as other vitrification tasks that are encountered in the future.

In addition, the needs consist of the following items:

1. Determination on a small-scale basis that glass-forming additives can vitrify the HLW to a form that has physically and chemically acceptable properties for repository storage.

2. Perform pilot scale melter tests that will provide the data necessary to establish process operating parameters and feed pretreatment requirements of the vitrification system. Also, these pilot scale tests will provide the data needed to verify that the candidate vitrifying formulations are processable.

3. Establishment of an administrative system to collect information and data that proves the suitability of the vitrified product for repository storage.

Technical Disposition: The TFA responded to this need within the following technical response(s):

- A9768, Specify and Enhance Design of HLW Glass Melters, TFA priority \#3.

- A9773, Improve Waste Loading in HLW Glass, TFA priority \#5.

- AA7S1, Durability of Multiphase Waste Glasses, TFA strategic task.

- AA7S2, New Melter Technology, TFA strategic task.

Site Need ID: ID-2.1.62

Site Need Title: Acceptance Criteria for Bin Set Closure

Need Summary: Bin set closure acceptance criteria are needed as soon as possible at INEEL so that technologies needed to achieve final closure can be determined. Bin set closure must consider RCRA requirements, NRC requirements, and the Settlement Agreement. Although the Settlement Agreement implies that "all calcined waste" must be removed from the bins, it is likely that the risk to the environment from some residual amount of calcine in the bins will be less than the risk of removing it. Bin set closure is similar to any HLW closure in the sense that the goal is to minimize the risk of releasing hazardous or radioactive material to the environment. Savannah River and Oak Ridge sites have experience with tank closure. Any licensing and operating experience or lessons learned from these sites are needed.

Assumption: It is assumed that final closure of a bin set would require filling bin voids with grout after removing this material.

Technical Disposition: The TFA responded to this need within the following technical response(s):

- A9924, Tank Closure Criteria/Decision Support, TFA priority \#30. 
Site Need ID: ID-2.1.64

Site Need Title: Solid-Liquid Separation Equipment Development and Application

Need Summary: Crossflow filtration has been tested at INTEC, and other sites around the Department of Energy complex, and proved to be a viable method for solids removal.

However, due to the variations of undissolved solids (UDS) present in INTEC wastes and the lack of suitable solids characterization data, additional testing is required. Extensive testing is planned and necessary to envelope operating conditions and performance limitations.

Amenable filtration technologies must ultimately be tested with simulated tank wastes and dissolved pilot plant calcine slurries.

Technical Disposition: The TFA responded to this need within the following technical response(s):

- A9584, Cross-Flow Filtration, TFA priority \#22.

Site Need ID: ID-2.1.65

Site Need Title: Treatment/Disposition of Removed Tank Solids

Need Summary: Waste in the INEEL HLW tank farm contains both settled and suspended solids. Once separated from the liquid, the solids need to be treated and packaged for disposal. Treatment may include dewatering, drying, stabilization or combinations of these technologies. Data is needed to provide a basis for design of the treatment system.

Technical Disposition: The TFA responded to this need within the following technical response(s):

- A9709, Waste Treatment Process Flowsheet Model, TFA priority \#7.

Site Need ID: ID-2.1.66

Site Need Title: Treatment/Disposition of Spent Ion Exchange Resins

Need Summary: One ion exchange (IX) resin currently proposed for extraction of Cs from dissolved INEEL calcines is AMP-PAN. The current baseline process for handling spent AMP-PAN resin is to dissolve the AMP sorbent (containing the Cs) with caustic and vitrify the eluent. The organic PAN substrate which remains would then be sluiced out of the column and grouted with other low activity waste (LAW) from separations processing. The impacts of the PAN on the quality and performance of the grout have not been evaluated. Test data is needed to determine whether the PAN will negatively impact the structural or leaching characteristec of performance grouts being considered for LDR-compliant disposal of LAW.

Technical Disposition: The TFA responded to this need within the following technical response(s):

- A9719, Conditioning and Immobilization of Low-Activity Waste, TFA priority $\# 14$.

- A9768, Specify and Enhance Design of HLW Glass Melters, TFA priority \#3.

- A9773, Improve Waste Loading in HLW Glass, TFA priority \#5.

- AA7S1, Durability of Multiphase Waste Glasses, TFA strategic task.

- AA7S2, New Melter Technology, TFA strategic task.

Site Need ID: ID-2.1.67

Site Need Title: High Level Waste Slurry Handling 
Need Summary: A number of processes currently being considered for treatment of HLW involve handling of slurries. Three such processes under consideration at the INEEL are as follows: 1) Removal of sludges in tank farm heels, 2) Transport and storage of undissolved solids from filtration of radioactive liquids (including existing sodium bearing liquid wastes and solutions from dissolution of calcined waste prior to liquid/liquid extraction of TRU, Cs, and $\mathrm{Sr}$ ), and 3) sluicing of resin materials into and out of IX columns for extracting soluble species (e.g., Cs, Tc, etc.). Systems will be needed to pump, convey, store, and retrieve these radioactive slurries. Performance data for such systems is needed in order to select and size appropriate equipment to handle the slurries that are likely to be handled in the course of waste processing. In addition, rheological measurements on specific slurries to be handled will be needed to predict equipment performance during design activities.

Technical Disposition: The TFA responded to this need within the following technical response(s):

- A9278, Slurry Transfer and Tank Waste Mixing Monitors, TFA priority \#35.

- A9361, Heel Retrieval from Obstructed Tanks, TFA priority \#16.

- A9365, Waste Transfer Pumping, TFA priority \#8.

Site Need ID: $\mathrm{ID}-2.1 .68$

Site Need Title: Technetium Removal from INEEL High Level Waste

Need Summary: Calcine stored in bins at the INEEL CSSF contains approximately $190 \mathrm{~kg}$ of Tc. Processing the calcine by dissolution and solvent extraction separations processes, as now configured, results in a high proportion of Tc being partitioned to LAW streams. The Tc in these wastes, when denitrated and grouted, will approach or exceed the NRC Class A limit for Tc. Technology is thus needed to remove Tc such that it can be immobilized with othe HAW streams into a glass waste form, and permit disposal of LAW as Class A wastes. Solvents used in the separations processes, namely the TRUEX and SREX processes, are known to remove Tc. Testing of these extraction processes to date had not focused on ways to optimize extraction of Tc or to strip Tc from the solvents. Alternative methods of removal of Tc may also be possible, i.e., IX or volatilization and collection of gaseous forms of Tc. Technical Disposition: The TFA responded to this need within the following technical response(s):

- A9501, INEEL Integrated Radionuclide Separations Process, TFA priority \#24.

Site Need ID: ID-2.1.69

Site Need Title: Solids Waste (Calcine) Retrieval from CSSF1

Need Summary: Highly radioactive waste material is being stored in bins in the First Calcined Solids Storage Facilities (CSSF1). The material was in the form of free granular solids and fines when it was sent to storage and is still expected to be free flowing. Systems are needed to retrieve the calcined solids out of CSSF1 and transfer them to a processing facility to be processed into an even more stable waste form or to another storage facility because CSSF1 is not seismically qualified.

Technical Disposition: The TFA responded to this need within the following technical response(s):

- A9331, Dry Solid Waste Retrieval, TFA priority \#31. 
Site Need ID: ID-2.1.70

Site Need Title: Low-Activity Waste Biodegradation Test

Need Summary: The new DOE Order 435.1-1 requires LLW to not produce toxic gases or structurally fail due to radiolysis or biodegradation (DOE M 435.1-1, Section IV.G(1)(d)4). The implementation guide (DOE G 435.1-1, page IV-47) does not provide further information on radiolysis or biodegradation testing. Specific requirements or guidelines are needed for the following: 1) what are the circumstances that require radiolyis and biodegradation tests, 2) what are the specific tests to be run, and 3) what are the pass/fail criteria?

Most DOE sites are using a cement/slag type grouting process for immobilizing LLW. Thus, this need applies to all DOE sites and laboratories in meeting the requirements for LLW disposal.

Technical Disposition: The TFA responded to this need within the following technical response(s):

- AA902, Data and Testing to Satisfy DOE Directive 435.1. Given that existing Nuclear Regulatory Commission guidance on biodegradation and radiolysis testing is an acceptable approach for satisfying DOE Order 435.1, the TFA plans no additional effort to respond to this need.

Site Need ID: ID-2.1.71

Site Need Title: Grout/Heel Mix in Place System

Need Summary: In view of the high solids volume, alternative processes for tank closure should be developed and evaluated. A primary alternative identified for TFF incorporates mixing grout with the tank heels in place. Although ORR has closed tanks by mixing in place, the conditions of the TFF and the characteristics of the tanks are unique. Development and testing of this alternative is essential as an option to the base process. This mock-up would incorporate bench top and full scale development and testing of systems to mix grout with the waste heel and bind the residuals. This development effort would include grout qualification testing in accordance with DOE 435.1.

Technical Disposition: The TFA responded to this need within the following technical response(s):

- A9985, Demonstration of Grout Injection Technology for Tank Closure, TFA priority \#40.

Site Need ID: ID-2.1.72

Site Need Title: Alternate Heel Sampling Systems

Need Summary: Develop and test an alternate (simplified) sampling system for retrieving samples from TFF tanks and vault sumps. There may be situations where the LDUA cannot support sampling efforts during closure activities due to conflicts or space/weight allocation over the tanks. The LDUA cannot sample vault sumps, and the existing vault sampling systems are not functional. Vault samples could be required in support of closure activities. Technical Disposition: The TFA responded to this need within the following technical response(s):

- AA1S1, Pre-Closure Interim Tank Maintenance, TFA strategic task.

- AA203, Residual Waste Sampling, TFA priority \#18. 


\section{Site: ORR}

Site Need ID: ORTK-01

Site Need Title: Tank Waste Characterization

Need Summary: Waste storage tanks must be emptied, and the tanks must be characterized for closure or returned to active service. Characterization technologies are needed at ORR to determine the quantity of sludge in the tanks before and after emptying. Characterization technologies are also needed to determine the structural integrity of the tanks if they are to be returned to long-term service. Routine structural integrity verification is required to keep the tanks in service.

Technical Disposition: The TFA responded to this need within the following technical response(s):

- A9143, HLW Tank Corrosion Control and Monitoring, TFA priority \#1.

- A9175, Tank Integrity Inspection Techniques, TFA priority \#11.

- AA1S1, Pre-Closure Interim Tank Maintenance, TFA strategic task.

Site Need ID: ORTK-02

Site Need Title: Tank Solid Waste Retieval

Need Summary: Vertical concrete storage tanks at ORR must be remediated. Process heels, hard sludge, and debris from the inside of old concrete storage tanks must be removed in order to remediate the tanks. Concrete walls which are contaminated from contact with radiological materials must be cleaned.

Technical Disposition: The TFA responded to this need within the following technical response(s):

- A9359, Waste Mixing and Retrieval, TFA priority \#34.

- A9367, Unobstructed Tank Heel Retrieval, TFA priority \#26.

- A9382, Horizontal and Small Tank Sludge Mixing and Retrieval, TFA priority \#27.

- AA3S2, SST Retrieval from Potential Leaking Tanks, TFA strategic task.

Site Need ID: ORTK-04

Site Need Title: Sludge Mixing and Slurry Transport

Need Summary: A system to transport bulk quantities of sludge from Oak Ridge National Laboratory (ORNL) underground tanks through miles of pipeline to consolidation tanks and treatment facilities is needed. Monitoring of the retrieved sludge is required to eliminate plugging and ensure slurry content.

Technical Disposition: The TFA responded to this need within the following technical response(s):

- A9278, Slurry Transfer and Tank Waste Mixing Monitors, TFA priority \#35.

- A9554, Hanford Tank Waste Chemistry, TFA priority \#4.

- AA3S1, Chemical Cleaning, TFA strategic task.

Site Need ID: ORTK-05

Site Need Title: Tank Sludge and Supernatant Separations 
Need Summary: There is a need to manage the excess water generated during sludge retrieval operations. Sludges and supernate/sluice water must be separated in a fast, costeffective manner during waste transfer and treatment operations.

Technical Disposition: The TFA responded to this need within the following technical response(s):

- A9555, Sludge Washing and Dissolution, TFA priority \#9.

- A9586, CIF Evaporator, TFA priority \#2.

- AA3S1, Chemical Cleaning, TFA strategic task.

- AA5S1, Removal of Key Non-Radioactive Elements from Tank Waste, TFA strategic task.

Site Need ID: ORTK-06

Site Need Title: Tank Sludge Supernatant Immobilization

Need Summary: The baseline plan for concentration and treatment of Oak Ridge National Laboratory (ORNL) tank waste is to remove Cs from the supernate by IX and grout the waste for disposal at the Nevada Test Site (NTS) and to solidify sludge for disposal at NTS or the Waste Isolation Pilot Plant (WIPP). Pretreatment may be required to meet the feed envelope needed by immobilization vendor. Waste form development is required to meet land disposal restriction (LDR) requirements.

Technical Disposition: The TFA responded to this need within the following technical response(s):

- A9719, Conditioning and Immobilization of Low-Activity Waste, TFA priority \#14.

Site Need ID: ORTK-09

Site Need Title: Tank Closure

Need Summary: Old deteriorating waste storage tanks exist which contain sludge heels that have been determined to be of negligible risk to health, safety, and environment. However, it will be very costly to remove the waste from tanks with limited access ports. Residual waste in the concrete walls and liners of the waste tanks may also dictate the need for tank closure. A technology is needed to in situ stabilize these sludge heels as a part of tank closure. Fill material which can meet acceptance criteria for tank closure is also required. Pre- and postclosure monitoring are needed.

Technical Disposition: The TFA responded to this need within the following technical response(s):

- A9923, Enhanced Grout Formulations for Tank Closure, TFA priority \#10.

- A9985, Demonstration of Grout Injection Technology for Tank Closure, TFA priority $\# 40$.

Site Need ID: ORTK-11

Site Need Title: Tank Supernatant Pretreatment

Need Summary: The baseline plan for treatment of Oak Ridge National Laboratory (ORNL) tank waste is to remove cesium from the supernate by ion exchange, evaporate to remove excess water, and grout the waste for disposal at the NTS. However, pretreatment to remove certain radionuclides and/or to reduce the volume of high-activity transuranic (TRU) waste may be required. 
Technical Disposition: The TFA responded to this need within the following technical response(s):

- A9570, Salt Disposition, TFA priority \#6.

- A9586, CIF Evaporator, TFA priority \#2.

\section{Site: SRS}

Site Need ID: SR00-1011

Site Need Title: Demonstrate Evaporation Technologies to Reduce Generation of Secondary Waste Volume from Consolidated Incineration Facility

Need Summary: An evaporation system is needed to significantly increase the TDS and TSS concentrations in the APCS quench water prior to discharge as secondary waste at SRS. This will reduce secondary waste generation and improve CIF treatment capacity.

Technical Disposition: The TFA responded to this need within the following technical response(s):

- A9586, CIF Evaporator, TFA priority \#2.

Site Need ID: SR00-2027

Site Need Title: Demonstrate Alternative Filtration Technologies to Replace HEPA Filters Need Summary: An alternative filtration technology, such as a HEPA filter constructed of washable media such as sintered metal or ceramic, will provide a HEPA filter which is not subject to water damage, and can be installed with built in water jets which will be used to wash the filter to reduce radiation and to eliminate to dirt accumulation.

Technical Disposition: The TFA responded to this need within the following technical response(s):

- A9171, Alternative Air Filtration Technology, TFA priority \#15.

Site Need ID: SR00-2028

Site Need Title: Alternative Waste Removal Technology

Need Summary: Conventional waste removal techniques using $150 \mathrm{HP}$ slurry pumps are considered costly and overly invasive. As a follow-on to extensive alternate mixing equipment (Flygt Mixer) testing in FY98 and 50HP Flygt Mixer deployment in FY99, additional Flygt Mixer development, testing and deployment is underway in FY00. The focus of this follow-on Flygt Mixer program will include evaluations of mixer sizing and operational strategies for salt dissolution for salt removal. Testing will determine the deployment operational strategies and orientation for mixing in Type I, II, and III tanks at SRS that contain cooling coils and other physical obstructions.

Technical Disposition: The TFA responded to this need within the following technical response(s):

- A9359, Waste Mixing and Retrieval, TFA priority \#34.

- A9362, Salt Cake Dissolution Retrieval, TFA priority \#19.

- AA3S1, Chemical Cleaning, TFA strategic task.

- AA3S2, SST Retrieval from Potential Leaking Tanks, TFA strategic task.

Site Need ID: SR00-2029

Site Need Title: Alternate DWPF Canister Decon Technology 
Need Summary: DWPF canister decontamination is a water-frit slurry blast technique that removes contamination and oxides from the entire canister exterior surface. The waste from this process is in two forms. An off-gas is routed to the facility vessel ventilation system and on to facility controlled ventilation exhaust. A water-frit slurry waste stream is pumped into the facility chemical process and fed into the vitrification process stream, to minimize liquid waste production. This coupling of canister decontamination with chemical processing is less than optimum and could limit production rates in the future.

Technical Disposition: The TFA responded to this need within the following technical response(s):

- A9772, Alternative HLW Canister Decontamination Techniques, TFA priority \#38.

Site Need ID: SR00-2031

Site Need Title: Develop Remote Technology to Improve DWPF Operations Need Summary: The DWPF is limited in the ability to perform remote maintenance, inspection, and cleanup activities within the shielded facility (canyon). The only access to the majority of the facility for maintenance, etc. is via overhead crane using hooks and an impact wrench. Viewing capability within the facility is limited to video cameras mounted on the Main Process Cell (MPC) Crane. It is desirable to develop improved capabilities to inspect, perform maintenance, and perform dencontamination/cleanup activities within the facility.

Technical Disposition: The TFA responded to this need within the following technical response(s):

- A9374, Remote Technologies for Process Cell Operations and Maintenance, TFA priority \#42.

Site Need ID: SR00-2032

Site Need Title: Optimize Melter Glass Chemistry and Increase Waste Loading Need Summary: DWPF complies with WAPS and process control requirements by demonstrating, to a high confidence, that melter feed will produce glass meeting all quality and processing requirements. This method requires that uncertainties associated with sampling, sample analysis and models used to estimate properties be determined and that sufficient allowance is made for these uncertainties when controlling feed composition. The existing model for liquidus temperature contains a large uncertainty associated and its application has led to reduction in allowable waste loading. Some constraints on the application of the durability model can cause acceptable feed batches to be rejected, because the durability is indeterminate (i.e., the applicability of the model is not certain).

Technical Disposition: The TFA responded to this need within the following technical response(s):

- A9773, Improve Waste Loading in HLW Glass, TFA priority \#5.

- AA7S1, Durability of Multiphase Waste Glasses, TFA strategic task.

- AA7S2, New Melter Technology, TFA strategic task.

Site Need ID: SR00-2033

Site Need Title: Provide Alternative Processing and/or Concentration Methods for DWPF Recycle Aqueous Streams 
Need Summary: At design rates, DWPF recycles about $7.5 \mathrm{gpm}$ aqueous stream to the tanks farms for evaporation. The stream consists of the following average composition: $\mathrm{H} 2 \mathrm{O}-$ 94.7\%; $\mathrm{NaOH} 4.0 \%$; NaNO3 0.3\%; NaNO2 0.6\%; NH3 300 ppm; misc. inorganics $0.3 \%$; misc. organics $700 \mathrm{ppm}$. In addition, the stream contains sludge solids and glass particulates from melter offgas fines and from process sample recycle. The gamma curie content for sludge-only operation is $7 \times 10-4$ curies/gal, primarily Cs-137. Incremental cost of processing this material in the Tank Farm is 78 cents/gal, not including salt disposition costs. However, if salt disposition does not start up on schedule at reasonable attainment levels, tank farm storage capacity in new style tanks will become critical and may cause DWPF to stop operations.

Technical Disposition: The TFA responded to this need within the following technical response(s):

- A9566, Vitrification Recycle, TFA priority \#29.

Site Need ID: SR00-2034

Site Need Title: Second Generation Salt Feed Preparation

Need Summary: For both salt disposition alternatives, there are significant Science and Technology (S\&T) questions and issues which must be answered to complete the design and construction activities in a time frame which allows HLW tank decommissioning in accordance with compliance agreements with the State of South Carolina and the Environmental Protection Agency (EPA). These technology assurance issues must be addressed in concert with the overall SRS Salt Disposition Project activities.

Science and technology is needed to support design and construction in the following three basic categories:

- Process chemistry

- Process engineering

- HLW System interface

Process chemistry information is needed for thermal and hydraulic transport properties, reaction kinetics and mass transfer properties necessary to finalize the conceptual planning and begin detail design. Examples of key decisions resulting from these activities include selection of tank mixing technology, selection of filtration technology and finalization of the process flowsheet.

Process engineering data is needed from engineering scale tests during pre-conceptual and conceptual design. Confirming performance data needs to be developed during unit operations testing to support preliminary design. This includes issues of equipment sizing, specific equipment attributes, material of construction, operational parameters such as pressure drop, and requirements for temperature control.

Integrated pilot facility operations needs to be completed during Final Design to confirm operation under upset conditions to determine the limits of operation and recovery, the limits of feed composition variability, and confirm design assumptions. This testing directly supports development of operating procedures, simulator development and operator training. 
Additional development and testing will be completed during Conceptual Design to assure that the feed and product interfaces of the cesium removal process are maintained with the HLW Tank Farm, DWPF and Saltstone. The issues of concern are assurance of glass qualifications, waste feed blending and characterization and waste acceptance.

Technical Disposition: The TFA responded to this need within the following technical response(s):

- A9570, Salt Disposition, TFA priority \#6.

Site Need ID: SR00-2035

Site Need Title: Develop Advanced Techniques for Life Extension of High Level Waste Tanks and Piping

Need Summary: The following technologies are needed:

- Small roving equipment is needed to inspect the annulus space of older tanks.

- Photographic inspection equipment is needed that can be used to ensure the integrity of the waste transfer piping systems.

- Data archiving of video and inspection information is in need of updating.

- Tank material property data base and adoption of consensus code fracture methodology is needed to evaluate observed degradation.

- Capabilities to quantify actual thermal gradients experienced in concrete portions of HLW tank confinement structures.

- Remote repair equipment and techniques are needed to perform temporary repairs of degradation that adversely affects structural stability of HLW tanks.

Technical Disposition: The TFA responded to this need within the following technical response(s):

- A9175, Tank Integrity Inspection Techniques, TFA priority \#11.

- AA1S1, Pre-Closure Interim Tank Maintenance, TFA strategic task.

Site Need ID: SR00-2036

Site Need Title: Develop Improved HLW Melter

Need Summary: Improvements to the glass melting system are required to increase reliability of glass pouring behavior in future DWPF melters.

Technical Disposition: The TFA responded to this need within the following technical response(s):

- A9768, Specify and Enhance Design of HLW Glass Melters, TFA priority \#3.

- A9773, Improve Waste Loading in HLW Glass, TFA priority \#5.

- AA7S1, Durability of Multiphase Waste Glasses, TFA strategic task.

- AA7S2, New Melter Technology, TFA strategic task.

Site Need ID: SR00-2037

Site Need Title: Tank Heel Removal/Closure Technology

Need Summary: The bulk waste removal process results in Contaminated Large Equipment (CLE) that requires final disposal to support tank heel removal. Currently, SRS does not have an established disposal path. Many disposal options must be evaluated and disposal path(s) must be selected that cost-effectively comply with regulatory, characterization, CONOPS, transportation, and disposal requirements. Technology needs associated with disposal of CLE to support Tank Heel Removal include characterization techniques for Low 
Level Waste, Hazardous/Mixed Waste, and Transuranic Waste, tank top flushing and decontamination equipment, and tank top remote size reduction methods that incorporate improved sleeving, ventilation, shielding, and containment features.

SRS has identified potential applications for the prototype Advanced Design Mixer Pump (ADMP) that is currently undergoing full scale testing at TNX. Velocity testing, simulant runs, resonance studies, and structural support design are needed in preparation for a hot demonstration in a waste tank. SRS considers Tank 18 to be a viable candidate for the prototype ADMP hot demonstration.

The challenges to mechanical heel removal equipment offered by obstructed waste tanks (types I and II) and rapid settling solids continue to emphasize the need for chemical cleaning technology. Additional studies are needed to support a hot demonstration of chemical cleaning techniques. Chemical cleaning evaluations are also needed to demonstrate the potential for selective removal of radioisotopes that provide the greatest environmental impact, such as Tc-99. Sludge dissolution chemical recipes need to continue to be tested for oxidation and dissolution of Tc. Processes that combine chemical and mechanical cleaning techniques also need to be considered for in-situ pretreatment for stabilization and/or removal of selected radionuclides (Tc-99, Pu-239, and S1-79) that are the main dose contributors revealed by fate and transport modeling.

Development of a low cost remote crawler platform with high pressure water spray payloads continued in FY99. Additional payloads such as a remote pump suction device, a minimal water usage local sluicer, grinders, blades for sludge dislodgment, and other tools for hardened sludge removal need to be developed in FY01. A crawler-based sludge suction device becomes more attractive as heel removal and tank closure efforts move to high level waste tanks containing sludge contaminants with significantly higher dose potential than previously closed tanks. A successful crawler-based suction device would help meet the minimal residual contaminant level requirements for closure of these HLW tanks. A reliable hose/tether management system that supports contamination and ALARA requirements is needed. This system should be adaptable for various crawlers, payloads, and other remotely operated devices to support the various challenges of heel removal in SRS obstructed tanks.

Existing transfer paths from the old style SRS tanks are complicated and require dedicated, intensive operations support. Additionally, these decades-old transfer lines require significant, costly refurbishment to achieve compliance with current DOE and regulatory requirements for waste transfers. New waste transfer systems that meet today's requirements (double-containment, leak detection, shielding, NPH protection, etc) are needed to support heel removal operations. The new transfer systems are to be specifically designed to transport waste directly to the SRS pretreatment process facilities and avoid interim waste storage/mixing/pumping facilities.

Waste slurry real-time data is needed to improve the technical confidence that the pumping systems for sludge removal are sized and operated properly for the physical/rheological properties of the slurry. On-line monitoring of the waste slurry characteristics in the mixing- 
pumping-transferring system would support optimization of the system while helping to avoid line pluggage and other system inefficiencies and process disruptions.

Subsequent to waste removal, the Extended Sludge Processing (ESP) pretreatment process requires that solids settle out of the slurry for sludge washing prior to vitrification. Shearing associated with over agitation by slurry pumps can cause colloidal suspension that adversely impacts the ESP process. Better techniques to achieve low shear mixing such as improved slurry pump impellers are needed to reduce colloidal suspension. Additionally, slurry additives to reverse colloidal suspension need to be studied to support the ESP process.

Type I, II, and III waste storage tanks are equipped with an annular space between the primary containment wall and the secondary containment wall. Waste has leaked into the tank annulus of several waste tanks. Prior to closing the tank, the waste in the annuli must be characterized and retrieved. Ventilation ductwork routed around the bottom of each annulus limits access to the annulus floor. In some cases such as tank 16, waste has leaked into the ductwork. Characterization and retrieval technology must be developed for final disposition of these waste tanks.

In addition to annulus characterization and retrieval, Type I and II tanks with leak sites require new technology to prevent additional leakage of waste into the annulus during waste removal operations. The introduction of large volumes of water and months long mixing campaigns during waste removal have the potential to re-activate leak sites that have selfsealed or existed above the past waste liquid level. Chemical additives or other mechanical sealing techniques must be identified and evaluated to prevent additional contaminants from reaching the tank annuli.

Equipment and techniques must be developed to visually inspect and to obtain representative samples of the F and H Tank Farm evaporator-CTS systems. Upon characterization, residual waste removal must be accomplished to render the systems ready for closure. All sampling and cleaning systems must require minimal manpower for operations while addressing radiological issues associated with these highly contaminated, difficult to access structures. The $1 \mathrm{~F}$ evaporator-CTS system must be cleaned and closed prior to closure of the entire Tanks 17-20 4-pack area.

Technical Disposition: The TFA responded to this need within the following technical response(s):

- A9157, Tank Leak Mitigation, TFA priority \#20.

- A9175, Tank Integrity Inspection Techniques, TFA priority \#11.

- A9278, Slurry Transfer and Tank Waste Mixing Monitors, TFA priority \#35.

- A9352, Remote Systems for Pit Operations and Maintenance, TFA priority \#23.

- A9359, Waste Mixing and Retrieval, TFA priority \#34.

- A9363, Chemical Cleaning of Tanks, TFA priority \#17.

- A9365, Waste Transfer Pumping, TFA priority \#8.

- A9367, Unobstructed Tank Heel Retrieval, TFA priority \#26.

- A9382, Horizontal and Small Tank Sludge Mixing and Retrieval, TFA priority \#27.

- A9554, Hanford Tank Waste Chemistry, TFA priority \#4. 
- AA1S1, Pre-Closure Interim Tank Maintenance, TFA strategic task.

- AA203, Residual Waste Sampling, TFA priority \#18.

- AA204, Characterization Methods for Contaminated Large Equipment. Personnel at Hanford have already identified a suitable hydraulic fluid that should not cause problems if a leak requires flushing some fluid into a HLW tank. This hydraulic fluid is the Tellus brand of Shell hydraulic fluids. While no additional technical development is required to address this need, information about the commercial product will be made known to all appropriate DOE sites.

- AA303, Waste Retrieval from Confined Spaces, TFA priority \#44.

- AA3S1, Chemical Cleaning, TFA strategic task.

- AA3S2, SST Retrieval from Potential Leaking Tanks, TFA strategic task.

Site Need ID: SR00-2039

Site Need Title: Methods to Unplug Waste Transfer Lines

Need Summary: As the tank clean-out and decommissioning program becomes active at SRS, there is an increasing potential that the transfer lines, which are in place, will become plugged (unable to facilitate waste transfer from one tank to another or from waste tanks to the DWPF ITP, or Saltstone, etc.).

Technical Disposition: The TFA responded to this need within the following technical response(s):

- A9376, Waste Transfer Line Plugging Prevention and Unplugging Methods, TFA priority \#12.

- A9554, Hanford Tank Waste Chemistry, TFA priority \#4.

- AA3S1, Chemical Cleaning, TFA strategic task.

Site Need ID: SR00-2040

Site Need Title: Demonstrate Remote Decommissioning and Disassembly of High Level Waste Processing Equipment

Need Summary: SRS currently does not have the capability to decommission, size reduce, decontaminate, classify and dispose of failed, highly contaminated processing equipment. This includes failed high level waste glass melters, process vessels and process equipment. The current approach to dealing with this equipment is long term storage in the canyon facilities, on regulated storage pads or in underground "Failed Equipment Storage Vaults." While storage is acceptable for the short term, technology must be developed to properly dispose of this equipment. This should include dismantling/size reduction of the equipment, decontamination and recyling of as much material as possible, disposal of the majority of the material as low-level waste and disposal of remaining high level waste materials in a controlled repository or as a recycle stream to tank farms and ultimately the Defense Waste Processing Facility (DWPF).

This need does not apply just to SRS. It spans the entire DOE complex wherever highly contaminated equipment is utilized or generated. Robotic/telerobotic technology currently exists which is capable of remote disassembly and decontamination of large equipment. However, much work will be involved in adapting that technology to high level equipment disposal. We must address the need now to ensure that the technology is developed and 
demonstrated to support funding, design and construction of D\&D facilities for SRS as well as other DOE sites.

Technical Disposition: The TFA responded to this need within the following technical response(s):

- A9777, Remote Disassembly of HLW Melters and Other Processing Equipment, TFA priority \#13.

Site Need ID: SR00-2041

Site Need Title: Develop Advanced Mixing Technology

Need Summary: HLW Waste Tanks

Conventional waste removal mixing techniques at SRS using 150HP slurry pumps have left up to 40,000 gallons of residual sludge waste heels. New technology for alternate mixer pumps is needed for tank mixing service. Variations of nozzle configurations to vary discharge jet angles will be considered. Single discharge nozzles to create a stronger, concentrated jet to break up hardened sludge will require furhter evaluation. An effective mixing system may require the combination of various mixing tools.

New mixer pump designs require an extensive technology selection process, product conceptual design development, and scaled and full size prototype testing. New feature improvements over baseline mixers would include 1) canned, close coupled motor that minimizes vibration problems, 2 ) simplified deployment methods that avoid crane support, and extensive steel superstructure, 3) methodology for elevation changes to eliminate cumbersome containment can handling and extensive contamination and radiological controls, and 4) gas seals that avoid costly bearing water support systems.

Baseline mixer pump speed control uses a costly remote, permanent style adjustable frequency drive (AFD) unit with control package. A portable, re-useable AFD/control bank unit with required environmental control system is needed for future mixer pumps.

Technical Disposition: The TFA responded to this need within the following technical response(s):

- A9359, Waste Mixing and Retrieval, TFA priority \#34.

Site Need ID: SR00-2044

Site Need Title: In-Situ Technology for Waste Characterization

Need Summary: A tank-deployed sample and characterization system is needed to provide real time radionuclide data to support evaluation for compliance with the WAC for waste removal operations. Additionally, radiological properties and sludge volume must be determined for fate and transport modeling to evaluate tank cleanliness for compliance with the performance objective for tank closure.

Rheological properties of waste are needed to meet pumping system requirements for process efficiency. Instruments to measure weight percent solids in slurries are needed to optimize sludge waste removal processes. In-situ, real time measurement of weight percent solids and other rheological data (yield stress, particle size, etc.) will facilitate sludge-rich slurries to improve waste removal process efficiency. Additionally, accurate weight percent solids data will reduce the risk of transfer line pluggage that can occur with high sludge solids content in 
slurries. Data is needed from various elevations in the tank with a accuracy of $+/-2 \mathrm{wt} \% \%$ which requires density readings of $.001 \mathrm{~g} / \mathrm{ml}$. Instruments should fit through 8 " inspection ports, and waste cannot be pulled out of the tank containment. Characterization equipment should be able to last multiple years in a high radiation environment. Slurry monitor development work in FY00 will include testing to support a hot demonstration of a prototype device in FY01.

A remote non-intrusive method to identify obstructions buried in sludge is needed to replace the costly baseline technique of riser probing. Obstructions below tank risers as small as a buried cable must be located prior to waste removal equipment installation.

Sludge volume mapping is needed to determine the residual sludge quantity for evaluation of tank cleanliness for closure using fate and transport modeling. The volume mapping technique must be able to map sludge that is covered by liquid supernate.

Technical Disposition: The TFA responded to this need within the following technical response(s):

- A9278, Slurry Transfer and Tank Waste Mixing Monitors, TFA priority \#35.

- AA201, Sludge Mapping and Volume Estimates, TFA priority \#47.

- AA202, In-Situ Waste Characterization, TFA priority \#32.

Site Need ID: SR00-2045

Site Need Title: In-Situ Waste Tank Corrosion Probe

Need Summary: It is desirable to have a probe instrument which will provide a readout of the corrosion rate as well as the analytical content of the chemical species which affect corrosion in a high level waste tank. This probe would provide the following benefits:

1. Minimize manual sampling for corrosion species, thereby reducing analytical costs as well as operator exposure

2. Minimize quantity of inhibitors and associated costs needed for corrosion control

3. Enable defining the ability of the inhibitors to mix by measuring concentration in a vertical plane

Raman technology has demonstrated ability to determine molar content in real time, but there have been no known radioactive waste tank deployments. Electrochemical Noise (EN) technology has been previously deployed in a waste tank at Hanford, but not in conjunction with a species monitor. Installation of the combined corrosion probe may provide important defining information.

Technical Disposition: The TFA responded to this need within the following technical response(s):

- A9143, HLW Tank Corrosion Control and Monitoring, TFA priority \#1.

Site Need ID: SR00-2051

Site Need Title: Technology to Mitigate Effects of Technetium Under Tank Closure Conditions

Need Summary: A better understanding is needed of the chemistry of Tc during the waste generation processes, under the conditions of waste removal, and under the conditions after closure. This better understanding will provide a tool for estimating the Tc- 99 concentrations 
in waste tanks when waste removal is being planned, and will reduce the conservatism of Tc99 modeling. Also, a better understanding of the chemistry may suggest better ways to remove Tc-99 effectively from the waste tank. This data needs to be obtained through characterization and performance modeling.

Technical Disposition: The TFA responded to this need within the following technical response(s):

- A9588, Leaching and Treatment of Technetium for Tank Closure, TFA priority \#41.

- A9960, Sequestering of Contaminant Migration, TFA priority \#36.

Site Need ID: SR00-2052

Site Need Title: Aluminum Dissolution from HAW Sludge and Its Impact on Downstream Salt Processing

Need Summary: The ability to remove at least $75 \%$ of the aluminum oxide content of sludge, irrespective of the form of the aluminum oxide present, must be demonstrated for radioactive "high aluminum" sludge to meet present projections for the total number of waste glass canisters at SRS. Conditions that assure $\mathrm{NaAlO} 2$ generated from aluminum dissolution does not revert to an insoluble hydrous oxide during subsequent evaporation, storage as concentrated supernate or salt cake, dissolution and subsequent treatment must also be demonstrated. The fate of $\mathrm{NaAlO} 2$ through other downstream waste processes must be determined to confirm that the aluminum removed from sludge will eventually be diverted to processing and disposal as a component of saltstone.

Technical Disposition: The TFA responded to this need within the following technical response(s):

- A9555, Sludge Washing and Dissolution, TFA priority \#9.

- AA3S1, Chemical Cleaning, TFA strategic task.

- AA5S1, Removal of Key Non-Radioactive Elements from Tank Waste, TFA strategic task.

Site Need ID: SR00-2055

Site Need Title: Increase in Applicability/Efficiency of High-Level Waste Planning Tool Need Summary: The Chemical Process Evaluation System (CPES), and its associated chemical database, is used to support HLW system planning efforts and flowsheet evaluations. Conversion of this DuPont program to ASPEN+ and the addition of glass property models will allow a more widely supported program at SRS and across the DOE complex and faster system planning outputs.

The SRS High Level Waste System Flowsheet has been developed and refined using CPES. An efficient flowsheet tool is still needed during operations to support evaluation of waste tank blending scenarios, make flowsheet improvements, and generate necessary data for regulatory and other needs. CPES does not currently include the product acceptance models that are contained in the Product Composition Control System (PCCS) which is used to judge. DWPF melter feed acceptability.

The HLW flowsheet needs to be recoded in a more accessible, efficient system. ASPEN+ is a software tool that is more widely used and is recommended for this application. The unit 
operations for the HLW system must be included along with glass product and process models which are currently included in the Product Composition Control System (PCCS). The system should also be set up to allow efficient modification of product/process models to allow for evaluation of changes and applicability to other DOE sites.

Technical Disposition: The TFA responded to this need within the following technical response(s):

- A9709, Waste Treatment Process Flowsheet Model, TFA priority \#7.

Site Need ID: SR00-3022

Site Need Title: In-Situ Grouting and/or Retrieval of Waste from Underground Tanks (Formerly Used for the Storage of Radioactive Solvents)

Need Summary: Twenty-two inactive underground radioactive waste solvent storage tanks (S1-S22) located in the Old Radioactive Waste Burial Ground (ORWBG) 643-E at SRS are scheduled for closure under the FFA agreement.

Technical Disposition: The TFA responded to this need within the following technical response(s):

- A9923, Enhanced Grout Formulations for Tank Closure, TFA priority \#10.

- A9985, Demonstration of Grout Injection Technology for Tank Closure, TFA priority \#40.

Site Need ID: SR00-2049-S

Site Need Title: Technetium Chemistry Under Waste Removal Conditions

Need Summary: A better understanding of the chemistry of Tc and other significant waste contaminants is needed to improve waste removal from tanks in preparation for tank closure. Technical Disposition: The TFA responded to this need within the following technical response(s):

- A9588, Leaching and Treatment of Technetium for Tank Closure, TFA priority \#41.

Site Need ID: SR00-2050-S

Site Need Title: Fracture Toughness Properties for Carbon Steel Utilized for Nuclear Waste Containment Vessels

Need Summary: Fundamental research is essential to build a materials property database which includes fracture toughness properties. These properties are critical for the analysis of current structural integrity and life extension of nuclear waste containment vessels.

Technical Disposition: The TFA responded to this need within the following technical response(s):

- A9175, Tank Integrity Inspection Techniques, TFA priority \#11.

- AA1S1, Pre-Closure Interim Tank Maintenance, TFA strategic task.

Site Need ID: SR00-2053-S

Site Need Title: Develop an Alternative Sorbent to Replace Monosodium Titanate for $\mathrm{Sr}$ and Actinide Removal

Need Summary: New materials with improved $\mathrm{Sr}$ and actinide removal performance are needed to replace monosodium titanate. 
Technical Disposition: The TFA responded to this need within the following technical response(s):

- A9570, Salt Disposition, TFA priority \#6.

Site Need ID: SR00-2054-S

Site Need Title: Develop Improved Radiochemical Analysis for High Ionic Strength Samples

Need Summary: Fundamental research in analytical chemistry is needed to develop methodology to analyze high ionic strength samples without the attendant problems associated with dilution.

Technical Disposition: The TFA responded to this need within the following technical response(s):

- A9264, Improve Waste Analytical Methods, TFA priority \#37.

\section{Site: WVDP}

Site Need ID: OH-WV-902

Site Need Title: Decontamination of High-Level Waste (HLW) Canisters (WVDP-2-99) Need Summary: The vitrification of HLW at the WVDP has produced more than 240 canisters of HLW (with a limited number to be generated in the future) requiring disposal in a deep geologic repository. The canisters are currently stored in a shielded cell within the Main Process Building at the WVDP. Prior to transport off-site for continued interim storage or disposal, the outer surfaces of the canisters must be cleaned to remove radioactive contamination resulting from filling, and from storage in a contaminated environment. The decontamination process should produce a secondary waste stream that can be managed readily for packaging, storage, and disposal.

Technical Disposition: The TFA responded to this need within the following technical response(s):

- A9772, Alternative HLW Canister Decontamination Techniques, TFA priority \#38.

Site Need ID: OH-WV-903

Site Need Title: Vitrification Expended Material Processing (WVDP-3-99)

Need Summary: A tooling system is needed to segregate, size reduce, decontaminate, and package metallic materials removed from the Vitrification Facility at WVDP, which are contaminated with HLW glass or slurry. The HLW removed from the materials would be returned to the operating melter, which itself has a finite life. The remaining metallic materials also need to be converted to a disposable form. The various tools must be deployable remotely for use in a highly radioactive environment.

Technical Disposition: The TFA responded to this need within the following technical response(s):

- A9777, Remote Disassembly of HLW Melters and Other Processing Equipment, TFA priority \#13.

Site Need ID: OH-WV-904

Site Need Title: High Level Waste Tank Closure 
Need Summary: HLW tank closure options being considered at WVDP include tank removal and in-place stabilization. Technologies required for exhuming the tank may include remote decontamination equipment and dismantling equipment. Technologies required for tank stabilization closure plans may include grout mixing, delivery plans, and performance assessments.

Technical Disposition: The TFA responded to this need within the following technical response(s):

- A9950, Barriers for Tank/Disposal Facility Closure. Because the Subcon Focus Area infiltration barrier effort is satisfying site needs for a guidance document, the TFA did not develop a separate technical response. The TFA will revisit whether it should develop a response after Hanford has an opportunity to review the Subcon effort and submit revised needs to the TFA.

- AA310, Tank Decontamination and Dismantling, TFA priority \#45.

Site Need ID: OH-WV-905

Site Need Title: Retrieval of Tank Heels

Need Summary: A heel of residual waste solids will remain on the bottom of tanks 8D-1 and 8D-2 at WVDP following bulk mixing of the liquid and solid wastes and subsequent transfers of the resulting slurry. Retrieval of these heels from tanks 8D-1 and 8D-2 may be required to meet tank closure requirements. A more effective and efficient waste retrieval system is needed which will mechanically retrieve and transport waste solids from the tanks.

Technical Disposition: The TFA responded to this need within the following technical response(s):

- A9361, Heel Retrieval from Obstructed Tanks, TFA priority \#16.

- A9382, Horizontal and Small Tank Sludge Mixing and Retrieval, TFA priority \#27.

Site Need ID: OH-WV-906

Site Need Title: Radioactivity Measurement of High-Level Waste Tank Residuals Need Summary: During the final phase of waste removal operations at WVDP, the remaining radioactivity will need to be measured accurately to determine the appropriate clean-out technology required to reach final tank closure requirements. When most of the HLW and hazardous wastes are removed, the residual wastes remaining in the tanks will be in the form of sludge, diffused to corrosion products and adhered to tank internal support structures. Measuring both residual activity of the radioactive wastes and the remaining amount of hazardous waste material in the tanks are important to determine if tank endpoints have been met. Accurately measuring the remaining radioactivity levels of key radionuclides as set out in 10 CFR Part 61 and the amount of $\mathrm{Hg}, \mathrm{Cr}$, and other hazardous constituents is necessary. An investigation of advanced assessment technology and measurement methods is necessary.

Technical Disposition: The TFA responded to this need within the following technical response(s):

- AA202, In-Situ Waste Characterization, TFA priority \#32.

Site Need ID: OH-WV-907

Site Need Title: High-Level Waste Tank Interim Maintenance 
Need Summary: Subsequent to the end of HLW processing at WVDP, the tanks will contain residual waste that must be maintained in a stable configuration pending development of the final closure method. Interim maintenance would include methods for prevention of tank corrosion, monitoring the tank integrity, and implementing structural stability measures. Technical Disposition: The TFA responded to this need within the following technical response(s):

- A9175, Tank Integrity Inspection Techniques, TFA priority \#11.

- AA1S1, Pre-Closure Interim Tank Maintenance, TFA strategic task.

Site Need ID: OH-WV-908

Site Need Title: Decontamination of High-Level Waste Contaminated Equipment Need Summary: Methods are needed to decontaminate equipment removed from the tanks to Class $\mathrm{C}$ radioactivity levels during waste retrieval operations. Equipment could include items such as mobilization pumps, transfer pumps, and mechanical arms.

Technical Disposition: The TFA responded to this need within the following technical response(s):

- AA311, Long-Length Equipment Handling. There is not a specific technology need associated with the need statement. Rather, the request is for general information. Based on discussions between the TFA Retrieval TIM and WVDP, the TFA will prepare a full technical response to this need. The scope for the work related to this need can be found in TFA technical response \#A9352, under the SRS Pit Operations, Task B4, Specification for 299H Pit System. The Retrieval TIM will provide information to the site regarding equipment decontamination via the TIM's role to provide technical assistance.

Site Need ID: OH-WV-914

Site Need Title: Development of Grout for In-Situ Closure

Need Summary: A grout recipe is needed that includes reducing agents and sorbents for capturing and/or binding mobile radiological and chemical contaminants. The grout would also serve to stabilize residual salts in-place in the tanks. The grout would be used for in situ HLW tank closure and for building stabilization to preclude the release of contaminants to the environment when wastes are closed in place. The grout would also have the characteristics of being pourable/pumpable, possess structural strength, and readily excavated in the event that an alternative method of closure is developed.

Technical Disposition: The TFA responded to this need within the following technical response(s):

- A9923, Enhanced Grout Formulations for Tank Closure, TFA priority \#10. 


\section{Distribution}

No. of

Copies

\section{OFFSITE}

2 Office of Scientific and

Technical Information

Akgunduz, Nina

U.S. Department of Energy

Fernald Environmental Management Project 7400 Willey Rd.

Cincinnati, OH 45030

G. J. Bastiaans

Ames Laboratory, Iowa State University

125 Spedding Hall

Ames, IA 50011-3020

J. Bell

Bell Consultants, Inc.

137 Bowsprit Lane

Kingston, TN 37763

R. Bromm

Fluor Fernald

P.O. Box 538704, MSTP: 52-4

Cincinnati, OH 45253-8704

B. L. Burks

The Providence Group, Inc.

P.O. Box 23408

Knoxville, TN 37933-1408

L. D. Bustard

Sandia National Laboratories

P.O. Box 5800, MS: 0734

Albuquerque, NM 87185-5800
No. of

Copies

J. Carberry

DuPont

Experimental Station

Building 249/119

P.O. Box 80249

Wilmington, DE 19880-0249

G. A. Choppin

Chemistry Department

Florida State University

$600 \mathrm{~W}$. College Ave

Tallahassee, FL 32306-3006

J. Drake

U.S. Department of Energy

10282 Rock Springs Rd.

West Valley, NY 14171

P. G. Eller

Los Alamos National Laboratory

NMT-6 Advanced Technology Group

MS: E5-10

Nuclear Material Technology Division

Los Alamos, NM 87544

R. C. Erdmann

P.O. Box 922

Grass Valley, CA 95945

D. W. Geiser, EM-50

U.S. Department of Energy

19901 Germantown Road

Germantown, MD 20874-1298

J. A. Gentilucci

JAG Technical Services, Inc.

127 Savannah Drive

Aiken, SC 29803

Distr.1 
No. of

Copies

12 K. D. Gerdes

U.S. Department of Energy

Office of Science and Technology

19901 Germantown Rd.

1154 Cloverleaf Bldg.

Germantown, MD 20874-1290

J. Ginanni

U.S. Department of Energy

Nevada Operations Office

232 Energy Way

Las Vegas, NV 89030

T. S. Gutmann

U.S. Department of Energy

Savannah River Operations Office

P.O. Box A

Aiken, SC 29802

J. Harness

U.S. Department of Energy

P.O. Box 2001, MS EM-93

Oak Ridge, TN 37831-8620

W. Haslebacher

U.S. Department of Energy

National Energy Technology

Laboratory (NETL)

3610 Collins Ferry Rd.

Morgantown, WV 26507-0880

E. W. Holtzscheiter

Westinghouse Savannah River

Company

Savannah River Technology Center

Aiken, SC 29802

J. Jones

U.S. Department of Energy

Nevada Operations Office

232 Energy Way

North Las Vegas, NV 89030-4199
No. of

Copies

D. S. Kaback

Concurrent Technologies Corp.

99918 th St, Ste 1615

Denver, CO 80202

B. R. Kowalski

5720 CR 116

Hesperus, CO 81326

B. Lewis

Westinghouse Savannah River Company

Building 703-8C, Room 7

P.O. Box 616

Aiken, SC 29802

K. Lockie

U.S. Department of Energy

Idaho Operations Office

750 DOE Place

Idaho Falls, ID 83402

C. P. McGinnis

Oak Ridge National Laboratory

P.O. Box 2008

Oak Ridge, TN 37821-6273

C. S. Mims

U.S. Department of Energy

Oak Ridge Operations Office

P.O. Box 2001

Oak Ridge, TN $37830-8620$

J. P. Morin

Westinghouse Savannah River Company

P.O. Box 616

Aiken, SC 29802

J. R. Noble-Dial

U.S. Department of Energy

Oak Ridge Operations Office

P.O. Box 2001

Oak Ridge, TN 37830-8620

Distr.2 
No. of

Copies

K. G. Picha

U.S. Department of Energy EM-32

Office of Eastern Operations

19901 Germantown Road

343/TREV

Germantown, MD 20874-1290

R.K. Quinn

Battelle Albuquerque Office

801 University Blvd., SE Suite 102

Albuquerque, NM 87106

S. M. Robinson

Oak Ridge National Laboratory

P.O. Box 2088, MS: 6044

Oak Ridge, TN 37831-6044

W. W. Schulz

W2S Company, Inc.

12704 Sandia Ridge Place NE

Albuquerque, NM 87111

J. L. Swanson

1318 Cottonwood

Richland, WA 99352

L. L. Talvarides

Syracuse University

334 Hinds Hall

Syracuse, NY 13244

T. R. Thomas

Lockheed Martin Idaho Technologies Company

P.O. Box 1625 MSIN 3458

Idaho Falls, ID 83415-3423

M. C. Thompson

Westinghouse Savannah River

Company

Building 773-A, C140

P.O. Box 616

Aiken, SC 29802
No. of

Copies

J. Unterzuber

SAIC

555 Quince Orchard Rd., Suite 500

Gaithersburg, MD 20878

P. Valenti

West Valley Nuclear Services

MS-53

10282 Rock Springs Rd.

West Valley, NY 14171

J. H. Valentine

Lockheed Martin Idaho

Technologies Company

P.O. Box 1625

Idaho Falls, ID 83415-3100

G. Vandegrift

Argonne National Laboratory

Building 205

9700 South Cass Avenue

Argonne, IL 60439

J. S. Watson

Oak Ridge National Laboratory

P.O. Box 2008, MS: 6178

Oak Ridge, TN 37831-6178

T. E. Weber

6622 West Victoria

Kennewick, WA 99336

T. D. Welch

Oak Ridge National Laboratory

P.O. Box 2008, MS: 6273

Oak Ridge, TN 37831-6273

F. Woolley

1 Walker Court

Cambridge MA 02138 
No. of

Copies

\section{ONSITE}

6 DOE-RL

R. F. Brich

K8-50

M. J. Glasper

L. S. Mamiya

K8-50

K8-50

B. M. Mauss

E. J. Cruz

T. P. Pietrok

K8-50

H6-60

K8-50

Los Alamos National Laboratory

M. T. Terry

K9-91

CH2M Hill (CHG) Hanford Group, Inc.

J. O. Honeyman

G3-21

Numatec Hanford Corporation

P. W. Gibbons

H5-61

Waste Policy Institute

E. Dysland

S. S. Briggs
No. of

Copies

28 Pacific Northwest National Laboratory

R. W. Allen

K9-69

T. M. Brouns

K9-08

J. L. Buelt

B. A. Carteret

P7-41

K9-91

S. H. Chin

K1-39

J. L. Devary

K6-96

S. W. Gajewski

K8-15

R. L. Gilchrist

K9-91

T. L. Page

K9-18

L. R. Roeder-Smith

K9-59

J. C. Treadway

K9-69

P. A. Scott

K9-46

W. C. Weimer

J. H. Westsik

P7-27

S. J. Widener

K9-91

TFA Library (8)

K9-77

K9-69

Information Release Office (5)

K1-06

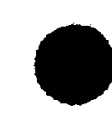

